\title{
Automated Pipelining for Clocked CMOS Logic and FPGAs
}

Thomas Michael Pepler, B. Eng.

A thesis submitted to the Faculty of Graduate and Postdoctoral Affairs in partial fulfillment of the requirements for the degree of

\section{Master of Applied Science}

in

Electrical and Computer Engineering

Ottawa-Carleton Institute for Electrical and Computer Engineering

Department of Electronics

Carleton University

Ottawa, Ontario

May, 2016

Copyright (C) 2016

Thomas Michael Pepler 


\section{Abstract}

Achieving multi-Gbps clock speeds with static CMOS logic requires migrating designs to smaller geometries, which present a higher development and fabrication cost. Instead, this research investigates the use of clocked CMOS logic to create an inherently pipelined circuit that can be clocked up to $2.5 \mathrm{x}$ faster than the standard cells available in the IC design kit. An algorithm was developed and implemented in Perl to process Verilog RTL netlists for compatibility with the clocked logic. As an example application, a cascaded integrator comb (CIC) filter for RF DSP was designed with the clocked CMOS and fabricated in the IBM $130 \mathrm{~nm}$ process. Unfortunately, due to an oversight with designing the boundary scan chain, true functionality of the circuit could not be verified. In another demonstration of concept, the algorithm was successfully applied to a QAM modulator design on a Xilinx Virtex-5 FPGA, which achieved a clock speed of $548 \mathrm{MHz}$. 
This thesis is dedicated to the memory of my grandfather, Stephen Pepler, who encouraged my passion for electronics. 


\section{Acknowledgments}

First, I would like to acknowledge my supervisor Prof. Calvin Plett, who supported me throughout this research and provided insightful comments on this thesis. As well, a large thank you goes to Mr. Yatish Kumar, who provided the initial concepts for this research, gave much-needed guidance on the technical issues, and proved to be a valuable mentor to me.

I am also grateful to Prof. Ryan Griffin and Mr. Stephen Toth for helping me with edits, and for encouraging me to finish.

Thank you to all the staff in the department office who helped me with everything administrative, and for being so kind and patient: Anna Lee, Blazenka Power, and Sylvie Beekmans. Thank you to Mr. Nagui Mikhail, who provided me with many of the resources I needed in testing (and especially for obtaining the FPGA evaluation board); as well as all the hard work he puts in to supporting our research. Thanks as well to Mr. Sean Follows, who helped me find the occasional bits and pieces for testing. I would also like to acknowledge Mr. Scott Bruce, who was patient and understanding of some of my odd inquiries about the Linux servers.

Thanks to the following people who helped me with the software and other parts of my design (in alphabetical order): Aaron, Behzad, Che, Jerry, Kimia, Michael, Nathan, Rachel, Raleigh, Richard, Ryan, Stephen, and Tyler. And thank you to Mr. Daniel Lanfranconi who helped me sort out some of the details with the place and route flow. To all the people in the blue room (which includes people not actually from the blue room), of which there are far too many to name, thank you for making my Wednesdays so "productive", and lunch times so enjoyable.

I would like to acknowledge CMC Microsystems for the provision of products and services that facilitated this research, including the CAD tools, fabrication services using the 130-nanometre CMOS technology from IBM, and test equipment loans. Also, I am grateful to the Province of Ontario, Carleton University, and other sources for providing funds through the Queen Elizabeth II Scholarship.

Finally, a huge thank you to my mother, Andrea, who supported me at home throughout the years; and to my father, John, who encouraged me to relax now and then. 


\section{Table of Contents}

$\begin{array}{ll}\text { Abstract } & \text { ii }\end{array}$

Acknowledgments $\quad$ iv

Table of Contents $\quad$ v

List of Tables $\quad$ vii

List of Figures viii

Abbreviations and Symbols $\quad$ xi

1 Introduction 1

1.1 Motivation . . . . . . . . . . . . . . . . . . 1

1.2 Objectives . . . . . . . . . . . . . . . 2

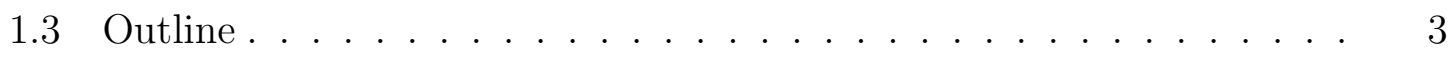

2 Background 4

2.1 Digital Signal Processing . . . . . . . . . . . . . . . . 4

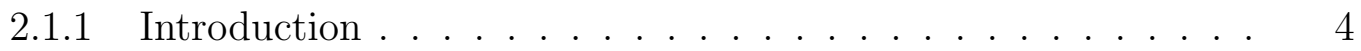

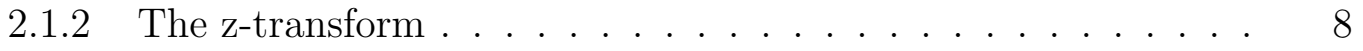

2.1 .3 Quantization .................... 11

2.2 Digital Filters . . . . . . . . . . . . . . . . . . . . . 12

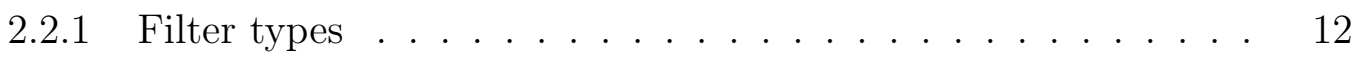

$2.2 .2 \quad$ CIC filters . . . . . . . . . . . . . . . . . . 13

2.2 .3 Polyphase FIR filters . . . . . . . . . . . . . . . 15

2.3 Graph Theory . . . . . . . . . . . . . . . . . . . . . . . . . . 19

2.3 .1 Introduction . . . . . . . . . . . . . . . . . . . . . 19

2.3 .2 Graph traversal . . . . . . . . . . . . . . . . . . . . . 21

2.4 RF Transmitters . . . . . . . . . . . . . . . . . . . . . . . 22

2.4.1 Quadrature amplitude modulation . . . . . . . . . . 22

2.4 .2 Error Vector Magnitude . . . . . . . . . . . . . . . . . 23 
2.4.3 Superheterodyne . . . . . . . . . . . . . . . . . 24

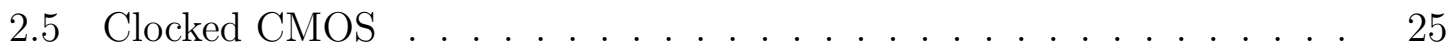

2.5 .1 Introduction . . . . . . . . . . . . . . . . 25

2.5 .2 Charge Sharing . . . . . . . . . . . . . . . . 27

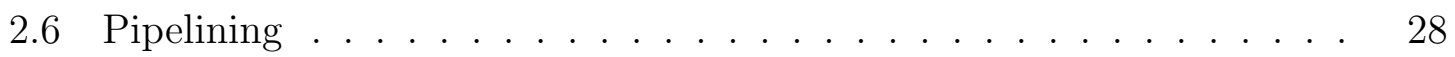

2.7 Summary . . . . . . . . . . . . . . . . . . . . . . . . 30

3 Design 31

3.1 Automatic Pipelining . . . . . . . . . . . . . . . . . . . 31

3.1 .1 Algorithms . . . . . . . . . . . . . . . . . 31

$3.1 .2 \quad$ Perl Script . . . . . . . . . . . . . . . . . . . 36

3.2 Integrated Circuit Cells . . . . . . . . . . . . . . . . . . 38

3.2 .1 Input/Output Buffers . . . . . . . . . . . . . . . . . . 39

$3.2 .2 \quad$ C $^{2}$ MOS Standard Cells . . . . . . . . . . . . . . . . . . 42

3.3 QAM Tx . . . . . . . . . . . . . . . . . . 45

3.3 .1 System Level Design _. . . . . . . . . . . . . . 51

3.3 .2 Behavioural Level Design . . . . . . . . . . . . . . . 56

3.3.3 Synthesis and FPGA Implementation . . . . . . . . . . 56

3.4 CIC Filter . . . . . . . . . . . . . . . . . . . 58

3.4 .1 System Level Design . . . . . . . . . . . . . . . . 58

3.4 .2 Behavioural Design . . . . . . . . . . . . . . . . . 58

3.4 .3 IC Design and Layout . . . . . . . . . . . . . . . . . 59

3.5 Summary . . . . . . . . . . . . . . . . . . . . . 62

4 Testing $\quad 64$

4.1 Buffers . . . . . . . . . . . . . . . . . . . . 64

4.2 CIC Filter . . . . . . . . . . . . . . . . . . 64

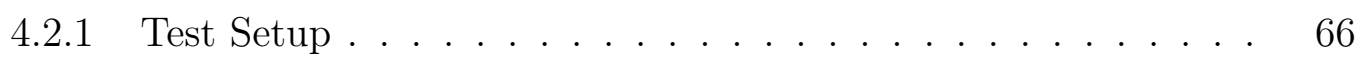

4.3 QAM Tx. . . . . . . . . . . . . . . . . 70

4.4 Summary . . . . . . . . . . . . . . . . . . . 72

5 Conclusion $\quad 74$

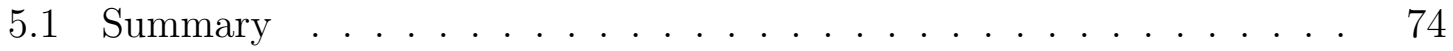

5.2 Contributions . . . . . . . . . . . . . . . . . . . 74

5.3 Future Work . . . . . . . . . . . . . . . . . . . . . 75

$\begin{array}{ll}\text { List of References } & 76\end{array}$ 


\section{List of Tables}

2.1 Some advantages and disadvantages of DSP over analog signal process-

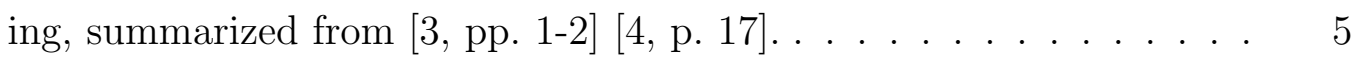

2.2 Table comparing Laplace and z-transform. . . . . . . . . . . . 9

2.3 A summary of characteristics of the two main DSP filter topologies. . 13

3.1 Summary of the IBM $130 \mathrm{~nm}$ kit parameters. . . . . . . . . . 38

3.2 Summary of the $\mathrm{C}^{2} \mathrm{MOS}$ standard cells and their transistor widths,

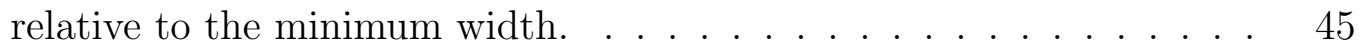

3.3 Summary of design parameters for QAM transmitter. . . . . . . . 55

3.4 A list of the pads used for the CIC chip. . . . . . . . . . . . 62 


\section{List of Figures}

2.1 Illustration of a signal being sampled in the time domain and the equivalent representation in the frequency domain. . . . . . . . 6

2.2 Illustration of how the s-plane maps to the z-plane. . . . . . . . 10

2.3 System block diagram of a CIC filter. . . . . . . . . . . . . 14

2.4 Frequency response of CIC filter. . . . . . . . . . . . . . . 14

2.5 System block diagram of a FIR filter. . . . . . . . . . . . 15

2.6 FIR filter impulse response. . . . . . . . . . . . . . . . . . 16

2.7 FIR filter polyphase responses. In this example, it can be seen that the 1st and 3rd set of coefficients are mirror image pairs, while the 2nd

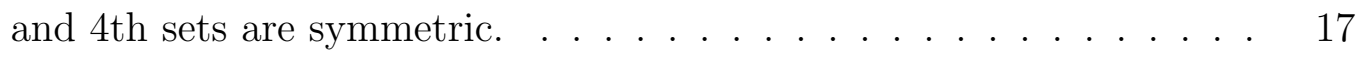

2.8 Polyphase FIR filter structure. . . . . . . . . . . . . . . . 18

2.9 A half adder circuit and its graph equivalent. . . . . . . . . 20

2.10 Example showing how a vertex can be both a child (for the red, nonbracketed relationship), or a parent (in the case with blue and brackets). 21

2.11 Example of a 16-QAM constellation, showing how the I and Q components add together to make a new phasor representing a sinusoid of the same frequency but with a different amplitude and phase shift. . . 23

2.12 Illustration of the various vectors used in the calculation of EVM. . . 24

2.13 Example of a superheterodyne transmitter. For simplicity the various amplifiers in the signal paths are not shown. . . . . . . . 25

2.14 Schematics of the two $\mathrm{C}^{2} \mathrm{MOS}$ gate styles. . . . . . . . . 26

2.15 Illustration of the charge sharing problem. While the clock transistors are off, the input changes, causing the charge to redistribute between

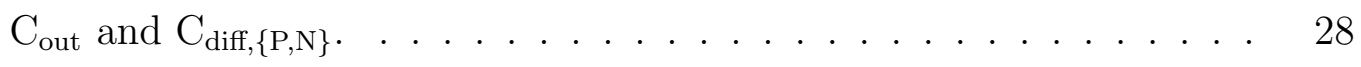

2.16 Schematics illustrating the use of pipelining. . . . . . . . . . 29

3.1 Illustration of an FPGA slice. . . . . . . . . . . . . . 33

3.2 An example circuit and its pipelined version. . . . . . . . . . 33 
3.3 Automatic pipelining and delay balancing algorithm. The DFS algorithm shown is reproduced from [10]. It is only conceptual, however, since the actual implementation in the Perl Graph module is based on a state-machine and hashes [10, p. 289], which is more efficient computationally. The setDepth and getDepth procedures are trivial, and not shown. . . . . . . . . . . . . . . . . . 35

3.4 An example circuit and its delay balanced version. . . . . . . . . 36

3.5 Delay balancing algorithm. . . . . . . . . . . . . 37

3.6 Schematic of the output buffer based on an inverter chain. . . . . . 39

3.7 Layout of the buffers test chip. . . . . . . . . . . . . 40

3.8 Schematic for the CML output buffer. . . . . . . . . . . . . . . 41

3.9 Layout for the CML output buffer. . . . . . . . . . . . . . 42

3.10 Schematic for the CML input buffer to drive the high-speed clock buffers. 43

3.11 Corresponding layout for the CML input buffer. The CML load resistors are arranged in a common-centroid configuration. . . . . . . . 44

3.12 Schematic and layout for the $\mathrm{C}^{2} \mathrm{MOS}$ buffer. . . . . . . . . 46

3.13 Schematic and layout of the $\mathrm{C}^{2} \mathrm{MOS}$ inverter. . . . . . . . . 47

3.14 Schematic and layout for the $\mathrm{C}^{2} \mathrm{MOS}$ NAND2 cell. . . . . . . . . . 48

3.15 Schematic and layout for the NOR2 $\mathrm{C}^{2} \mathrm{MOS}$ cell. $\ldots \ldots \ldots \ldots$

3.16 Layouts for the filler cells created. . . . . . . . . . . . . 50

3.17 System block diagram for the QAM transmitter. . . . . . . . . . 51

3.18 Symbol map for 16-QAM, adapted from the WiGig standard [31]. . . 52

3.19 An example of a raised cosine rolloff filter's frequency response. . . . 53

3.20 An example of the spectrum obtained for 1200 symbols worth of random bits, using the RRCF pulse shaping filter developed. Also shown is the transmit mask for WiGig [31, Fig. 21-1] . . . . . . . . . 55

3.21 The polyphase FIR filter, modified for coefficient symmetry. . . . . 57

3.22 System block diagram of CIC filter. . . . . . . . . . . . 58

3.23 Frequency response of the CIC filter that was designed. . . . . . . 59

3.24 Schematic for the fanout-of-4 clock buffer cell. . . . . . . . . . 60

3.25 Layout of the digital core of the CIC filter. . . . . . . . . . . 61

3.26 Layout of the entire CIC filter IC . . . . . . . . . . . . 63

4.1 Photomicrograph of the buffers chip. The size of the die is 1 by $1 \mathrm{~mm}$. $\quad 65$

4.2 Photomicrograph of the CIC chip. The die size is 1 by $1.5 \mathrm{~mm}$. . . . 67

4.3 The CFP24 test jig PCB with the packaged CIC chip inserted. The relevant signals are labelled on their corresponding SMAs. . . . . . 68 
4.4 Laboratory bench setup for testing the CIC filter. . . . . . . . . . . 69

4.5 Schematic showing the test setup for the CIC filter. . . . . . . . . . . 70

4.6 Plot of the simulation of the scan chain signals, showing the much slower rise time of $c l k$ compared to $c l k_{b}$. . . . . . . . . . . . . . . . 71

4.7 Plot of the clock signal and all of the outputs of the scan chain FFs. . 71

4.8 Plot of the output of the QAM modulator (blue), and the ideal symbol values $($ red, with stems). . . . . . . . . . . . . . 72 


\section{Abbreviations and Symbols}

The following is a list of abbreviations and symbols used in this thesis, and their definitions.

$\begin{array}{ll}\text { A/D } & \text { Analog to Digital } \\ \text { ADC } & \text { Analog to Digital Converter } \\ \text { ASIC } & \text { Application Specific Integrated Circuit } \\ \text { CAD } & \text { Computer Aided Design } \\ \text { CIC } & \text { Cascaded Integrator-Comb } \\ \text { C } 2 \text { MOS } & \text { Clocked CMOS } \\ \text { CMOS } & \text { Complementary Metal-Oxide Semiconductor } \\ \text { CPAN } & \text { Comprehensive Perl Archive Network } \\ \text { CPU } & \text { Central Processing Unit } \\ \text { D/A } & \text { Digital to Analog } \\ \text { DAC } & \text { Digital to Analog Converter } \\ \text { DAG } & \text { Directed Acyclic Graph } \\ \text { DFT } & \text { Design for Test } \\ \text { DRC } & \text { Design Rules Check } \\ \text { DSA } & \text { Digital Signal Analyzer } \\ \text { DSP } & \text { Digital Signal Processing } \\ \text { DUT } & \text { Device Under Test } \\ \text { EM } & \text { Electro-magnetic } \\ \text { EV } & \text { Error Vector Magnitude } \\ \text { ENansistor }\end{array}$




\begin{tabular}{|c|c|}
\hline FF & Flip-flop \\
\hline FIR & Finite Impulse Response \\
\hline FPGA & Field Programmable Gate Array \\
\hline Gbps & Gigabit(s) per second \\
\hline GSG & Ground-Signal-Ground \\
\hline GSSG & Ground-Signal-Signal-Ground \\
\hline HDL & Hardware Description Language \\
\hline IC & Integrated Circuit \\
\hline $\mathrm{I} / \mathrm{O}$ & Input / Output \\
\hline IEEE & Institute of Electrical and Electronics Engineers \\
\hline IIR & Infinite Impulse Response \\
\hline LVS & Layout Versus Schematic \\
\hline MAC & Multiply and Accumulate \\
\hline MOSFET & Metal-Oxide Semiconductor Field Effect Transistor \\
\hline NMOS & N-type MOSFET \\
\hline PAPR & Peak-to-Average Power Ratio \\
\hline PMOS & P-type MOSFET \\
\hline PnR & Place And Route \\
\hline QAM & Quadrature Amplitude Modulation \\
\hline RF & Radio Frequency \\
\hline $\mathrm{RCF}$ & Raised Cosine-rolloff Filter \\
\hline SNR & Signal to Noise Ratio \\
\hline SQNR & Signal to Quantization-Noise Ratio \\
\hline VLSI & Very Large Scale Integration \\
\hline WiGig & Wireless Gigabit standard \\
\hline
\end{tabular}




\section{Chapter 1}

\section{Introduction}

\subsection{Motivation}

Speed, power, and area efficiency are three fundamental design goals for any circuit. For a digital synchronous circuit, speed is often addressed via pipelining. Contemporary pipelining is either a manual or semi-manual process wherein the designer manually edits the digital netlist to insert flip-flops (also called registers) at appropriate points in the data path, or adds extra flip-flops at the beginning or end of the data path and then uses a register balancing algorithm (also called retiming) to evenly spread the flip-flops between the input and output. The trade-off is that the extra flip-flops increase the circuit power consumption and require additional area.

All three design goals mentioned could also be improved by migrating a design to a smaller-geometry integrated circuit process (often called a die shrink). However, this jeopardizes the fourth (and often most important) design factor: cost. Despite the issue of cost, for the last few decades the aforementioned technique has been the norm, which has inspired (and self-fulfilled) the venerable Moore's Law. That being said, it has become readily apparent in the last decade that soon this technique will no longer be viable for a variety of reasons. The obvious problem is that the size of transistors are rapidly approaching the very size of the atoms of which they are built. Besides that problem, the second and more imminent problem is the limitation of the optics used to pattern the geometry from the layer masks. This second problem has led to the introduction greater numbers of layout design rules which must be accounted for, thus increasing the design time.

Any new technology that attempts to supplant silicon for the promise of increased circuit performance also has many hurdles to overcome. The reluctance of the industry to abandon investments in equipment for processing silicon wafers will delay the 
introduction of this new technology; unless of course it is already compatible with existing process flows and equipment.

It may be worthwhile, then, to re-examine techniques which have long been forgotten and abandoned due to the convenience of die shrinking ${ }^{1}$, and apply these to newer process geometries.

The first and last options are combined in this thesis in the form of an application specific integrated circuit (ASIC). The clocked CMOS logic cell introduced by Suzuki et al. in 1973 [1] is combined with pipelining to attempt the creation of a digital circuit which runs with a clock frequency of a few $\mathrm{GHz}$ in a relatively older $130 \mathrm{~nm}$ process.

The other trend in the industry is the division of the IC design from the functional design by using field-programmable gate arrays (FPGAs). The pipelining algorithm developed for the ASIC was modified slightly to apply it to an FPGA-based design.

\subsection{Objectives}

The objectives of this thesis were to develop an algorithm for automatically pipelining a register-transfer level digital design, and to demonstrate the technique with a CMOS ASIC and an FPGA. Additionally, the ASIC used the less conventional logic family known as Clocked CMOS to improve the area and power efficiency compared to the traditional pipelining approach of inserting flip-flops between logic stages.

In summary, the objectives were:

- Develop algorithms for automated pipelining or delay balancing of logic circuits.

- To demonstrate the functionality of the algorithms by applying it to a practical circuit that could be part of a radio transmitter. Specifically, the algorithms were applied to digital signal processing circuits.

- Use $\mathrm{C}^{2} \mathrm{MOS}$ logic instead of conventional pipelining to achieve faster data rates in an older CMOS process, with power and area still reasonably low.

- To show the viability of the pipelining algorithm for FPGA designs.

Although the algorithms could have been demonstrated with simpler circuits, it was decided that radio applications would be the best fit for the research group. As well, it was assumed that the best illustration of the usefulness of the algorithms was by developing practical circuits.

\footnotetext{
${ }^{1}$ Or even methodologies that pre-date integrated circuits
} 


\subsection{Outline}

The thesis will begin with a background chapter on the relevant theory used in the design of the algorithm, and the different circuits. Then the chapter following that will provide some detail on the design process and decisions made. The fourth chapter presents results from measuring the circuits in the laboratory. And finally, the thesis is summarized in the last chapter. 


\section{Chapter 2}

\section{Background}

This chapter will introduce the background theory that the design work is based on.

\subsection{Digital Signal Processing}

In this section the fundamentals of digital signal processing will be covered to aid in understanding some of the digital filter theory, as well as some of the choices made in the design of the digital filters used in this research.

\subsubsection{Introduction}

Digital signal processing (DSP) is concerned with the design, analysis, and use of algorithms, systems, software, and circuits for processing signals with digital hardware. The motivation for using digital hardware is that it can be simpler to design and made reconfigurable more easily than analog circuits; but possibly have greater power consumption. Table 2.1 on the following page summarizes the main advantages and disadvantages of digital versus analog signal processing. Typically, the digital hardware for a DSP system is one or a combination of the following: application specific integrated circuits (ASICs), digital signal processors (DSPs), or general purpose central processing units (CPUs) [2, Ch. 9].

\section{Sampling}

In many DSP systems the signals originate as analog, need to be output as analog, or both. The conversion of signals from analog to digital requires sampling the analog signal in time and with quantized amplitude levels, using a circuit known as an analogto-digital converter (ADC). Similarly, the digital values are converted back to analog with a digital-to-analog converter circuit (DAC), also at fixed samples in time and 
Table 2.1: Some advantages and disadvantages of DSP over analog signal processing, summarized from [3, pp. 1-2] [4, p. 17].

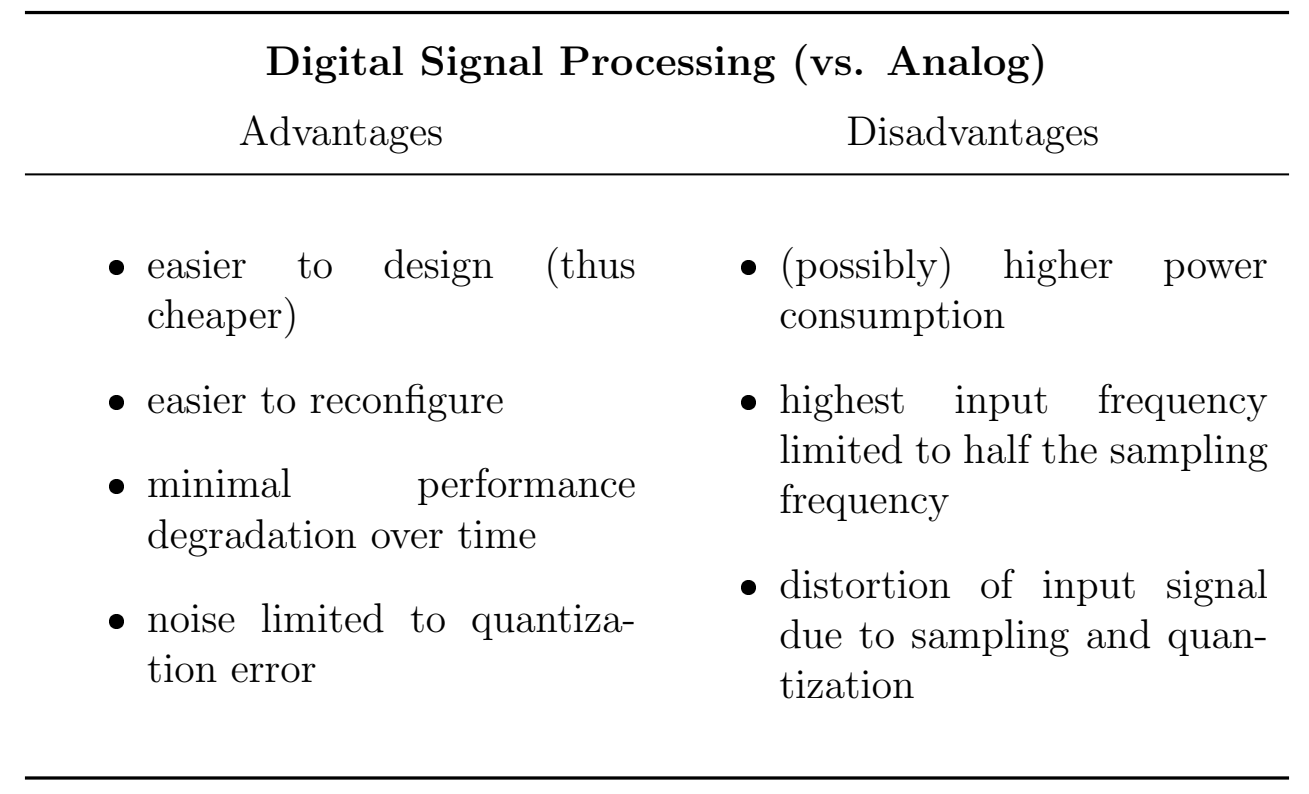

with quantized levels. ADCs and DACs sample at a rate determined by an input sampling clock signal.

To partly understand what effect sampling has on the signal, the Laplace transform is used to observe the spectrum. Sampling an analog waveform with a fixed frequency can be represented mathematically by multiplying the waveform with an infinite impulse train, with impulses spaced $T_{s}$ seconds apart (the period of the sampling clock). A convenient property of the Laplace transform is that it maps the multiplication of time domain signals into the convolution of their respective signal spectra. Thus the spectrum of any sampled analog signal is simply found by convolving its spectrum with the spectrum of an impulse train. The Laplace transform (spectrum) of an infinite impulse train is also an infinite set of impulses, but spaced $f_{s}=\left(1 / T_{s}\right) \mathrm{Hz}$ apart along the frequency axis. Therefore, the spectrum of the sampled signal is simply the spectrum of the original signal repeated every $f_{s}$ (see Figure 2.1 on the next page for a visual representation).

\section{Aliasing and imaging}

Two important points can be made by observing the spectrum of the sampled signal:

1. The spectrum of the original (unsampled) signal still exists at baseband, and can be recovered with simple low pass filtering, as long as the following is also true. 
Time Domain
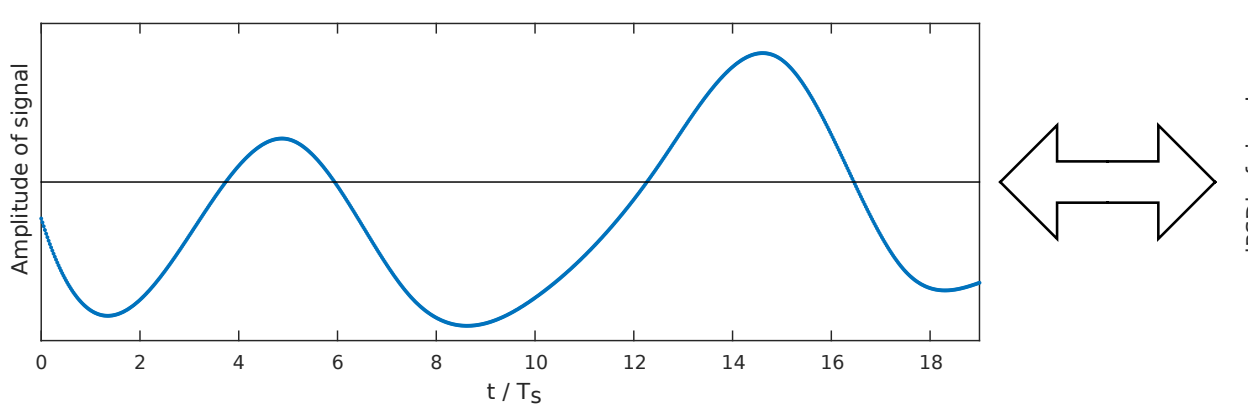

Frequency Domain
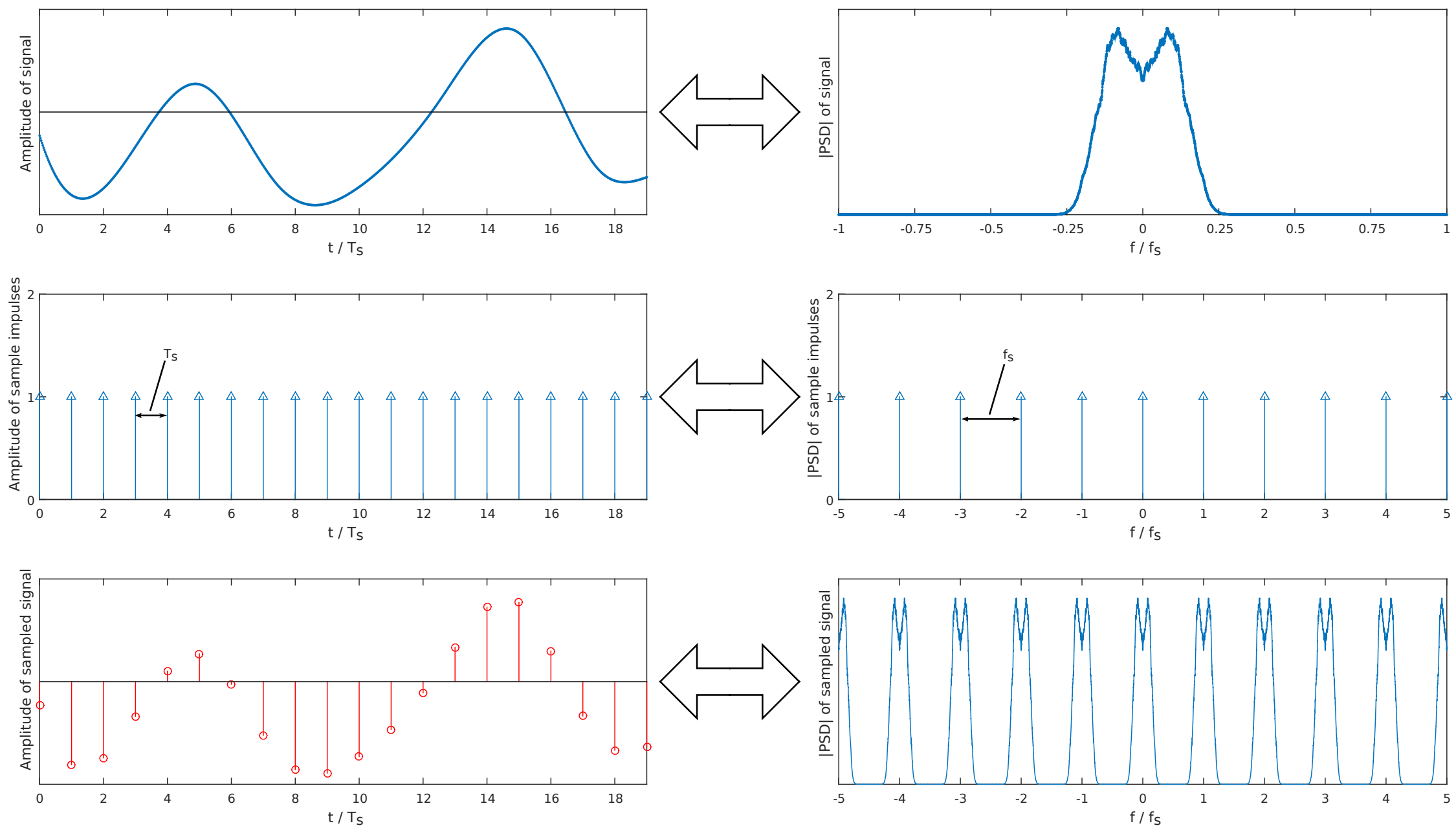

Figure 2.1: Illustration of a signal being sampled in the time domain and the equivalent representation in the frequency domain. 
2. The spectrum at baseband does not overlap with any of the spectra centred at multiples of $f_{s}$, which are called the aliases.

In other words, the highest frequency in the baseband (denoted $f_{B W}$ ) must be less than the lowest frequency in the first aliased spectrum ${ }^{1}$. The first aliased spectrum is centred at $f_{s}$, and the lowest frequency is $\left(f_{s}-f_{B W}\right)$. With some algebraic manipulation, an important relationship is derived:

$$
\begin{aligned}
f_{B W} & <\left(f_{s}-f_{B W}\right) \\
f_{s} & >2 f_{B W}
\end{aligned}
$$

Equation $(2.1)^{2}$ defines what is known as the Nyquist sampling rate, dictating the minimum sampling rate such that the original signal can be completely recovered. The fact that the signal can be completely recovered, as long as the sampling frequency meets the Nyquist sampling rate, is known as the sampling theorem [5, p. 40].

Conversely, digital-to-analog conversion will produce copies of the baseband spectrum at multiples of $f_{s}$, which are known as images. The signal can be recovered by using an appropriate analog anti-imaging filter to remove the images. ${ }^{3}$

\section{Decimation and interpolation}

Sample rate change is the process of converting a DSP signal at a given sample frequency to an equivalent signal at a higher or lower sample frequency. In some systems it is desirable to have the sample rate of the signal change at some point in the signal chain [6, Ch. 13]. For example, many systems operate the input ADC or output DAC at an oversampling rate (i.e. sample at a rate greater than the Nyquist rate) in order to relieve some of the analog anti-aliasing/imaging filter requirements (or other parts of the ADC or DAC, as is the purpose of a delta-sigma converter) [3, p. 774] [6, pp. 3-9]. However, to reduce circuit power consumption or area, the sample rate in the signal processing blocks can be reduced to the Nyquist sampling rate.

\footnotetext{
${ }^{1}$ Meeting this condition also guarantees that there is no interference from the higher aliases at $2 f_{s}, 3 f_{s}$, etc.

${ }^{2}$ The strict inequality $(>)$, rather than the non-strict inequality $(\geq)$, is required since there is ambiguity in the phase of the sampled signal at $f=\frac{1}{2} f_{s}$. E.g.: a sinusoid could be sampled at every zero crossing, producing all 0 samples.

${ }^{3}$ However, it is much easier to implement DACs which hold their output voltage at the last sample, which produces an output waveform that looks like the sideways view of a staircase. The spectrum of this type of signal has images which are significantly attenuated, thus not only is the DAC easier to implement, but the anti-imaging filter too.
} 
A decrease in sampling rate is known as decimation or down-sampling, and can be seen as a similar process to sampling an analog signal (i.e. A/D conversion). The analog signal can be thought of as a signal having an infinite sample rate which is then converted to a signal with some finite, and therefore lower, sample rate. With decimation, the sample rate is reduced by a factor $D$, which involves removing $D-1$ samples from the original signal; and just as with analog to digital sampling, some form of filtering (or smoothing of the waveform) must be taken to avoid any higher frequency signal components aliasing at the lower sampling rate.

An increase in sampling rate is known as interpolation or up-sampling. Just as decimation is analogous to $\mathrm{A} / \mathrm{D}$ conversion, interpolation is like digital-to-analog conversion. Interpolation is increasing the sample rate by a factor $L$ which is accomplished by inserting $L-1$ zero-valued samples between the original samples; this results in imaging of the original spectrum. The imaging is removed by some form of digital anti-imaging filter operation.

Often the up-/down-sampling is combined with the anti-imaging/-aliasing in a single process to achieve the interpolation/decimation. This also allows for some of the arithmetic operations for the filtering to be moved to the slower sampling side. Examples of this will be seen later in the sections on CIC filters (Section 2.2.2 on page 13) and polyphase FIR filters (Section 2.2.3 on page 15).

\subsubsection{The z-transform}

Analog systems may be analyzed and designed using the Laplace transform, whereas digital systems use the z-transform. Some of the key differences between these two transforms are illustrated in Table 2.2 on the next page.

However, the two transforms are not completely separate ideas, as it can be shown that one can be transformed into the other [5, pp. 605-610]. The variables for the $s$-plane and $z$-plane are related to each other as follows:

$$
\begin{aligned}
s & \equiv \sigma+j \omega \\
z & \equiv r e^{j \Omega} \\
r=e^{\sigma}, \quad \Omega & =\left.\omega\right|_{-\omega_{s} / 2} \leq \omega \leq \omega_{s} / 2
\end{aligned}
$$

Equation (2.2) and (2.3) indicate that the $s$-plane is rectangular in form while the 
Table 2.2: Table comparing Laplace and z-transform.

\begin{tabular}{|c|c|}
\hline Laplace Transform & z-transform \\
\hline signal is continuous in time & signal is discontinuous in time \\
\hline $\begin{array}{l}\text { maps time domain function to } \\
s \text {-domain }\end{array}$ & $\begin{array}{l}\text { maps time domain samples } \\
z \text {-domain }\end{array}$ \\
\hline $\begin{array}{l}\text { system response in the time domain has } \\
\text { differential equations }\end{array}$ & $\begin{array}{l}\text { system response to samples has differ- } \\
\text { ence equations }\end{array}$ \\
\hline $\begin{array}{l}\text { transform equation uses integration of } \\
\text { signal function }\end{array}$ & $\begin{array}{l}\text { transform uses summation of signal } \\
\text { samples }\end{array}$ \\
\hline $\mathcal{L}\{x(t)\}=X(s)=\int_{t=-\infty}^{\infty} x(t) \mathrm{e}^{-s t} \mathrm{~d} t$ & $\mathcal{Z}\{x[n]\}=X(z)=\sum_{n=-\infty}^{\infty} x[n] z^{-n}$ \\
\hline
\end{tabular}

$z$-plane is polar. Figure 2.2 on the next page shows how the $s$-plane morphs into the $z$-plane, and that the left-hand plane of the $s$-plane corresponds to the inside of the unit circle on the $z$-plane; and the right-hand plane maps to the region of the $s$-plane outside the unit circle.

The difference equation of a sampled system describes how the present output of the system is related to the present and past input, and the past output, samples; for a generic system it has the form shown in (2.4). It is the equivalent to the differential equation for a continuous time system.

$$
\begin{aligned}
y(n)= & a_{0} x[n]+a_{1} x[n-1]+a_{2} x[n-2]+\cdots+a_{k} x[n-k]+\cdots \\
& +b_{1} y[n-1]+b_{2} y[n-2]+\cdots+b_{k} y[n-k]+\cdots
\end{aligned}
$$

As with $s$-domain analysis, it is also useful to write a transfer function equation for a sampled system using the $z$-domain variable. By taking the z-transform of (2.4) and performing some algebraic manipulation, the z-domain transfer function is:

$$
\frac{Y(z)}{X(z)}=H(z)=\frac{a_{0}+a_{1} z^{-1}+a_{2} z^{-2}+\cdots+a_{k} z^{-k}+\cdots}{1-b_{1} z^{-1}-b_{2} z^{-2}-\cdots-b_{k} z^{-k}-\cdots}
$$

From (2.5) it can be seen that delaying any signal by $k$ samples has the effect of multiplying the original signal's z-transform by $z^{-k}$. For example, a delay by 1 sample multiplies the signal by $z^{-1}$, an element which often appears in $z$-domain system block diagrams. 

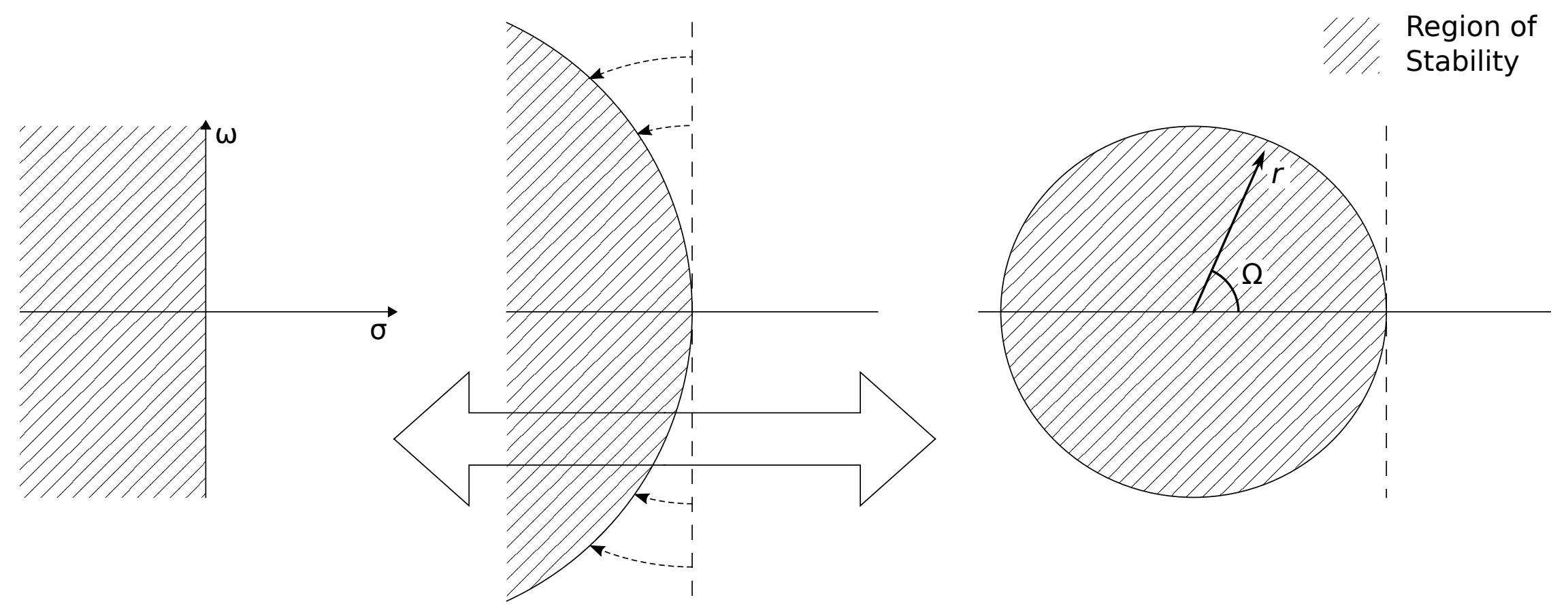

Figure 2.2: Illustration of how the s-plane maps to the z-plane. 
While the form of the $z$-transform in (2.5) is convenient for directly converting to the difference equation (and vice versa), the frequency response of the system is more easily determined by applying the partial fraction expansion to (2.5) and plotting the resulting poles and zeros on the $z$-plane.

Additionally, taking the inverse $z$-transform of the system transfer function $H(z)$, results in obtaining the system's impulse response in the time domain, $h(n)$.

\subsubsection{Quantization}

The other limitation of DSP systems is the finite number of amplitudes they can resolve. Whether a DSP system is fixed or floating point, there will be some maximum digital word width, and hence a finite amplitude resolution. The conversion of the continuously varying amplitude of an analog signal to the discrete amplitude levels for a digital word is known as quantization; and the difference between the analog and digital amplitude is known as the quantization error or quantization noise $\left(e_{q}[n]\right)[3$, pp. 33-34].

If a DSP system is $N$ bits wide, then it can represent $L=2^{N}$ different amplitude levels. The range $(R)$ is the difference between the highest and lowest amplitude level of the system; and the amplitude resolution $(\Delta)$ is given by (assuming the spacing between levels is constant):

$$
\Delta=\frac{R}{2^{N}}
$$

Assuming the desired signal lies within the range limits of the $N$ bit wide DSP system, then the quantization error for any $n$-th sample is bounded as follows:

$$
-\frac{\Delta}{2} \leq e_{q}[n] \leq \frac{\Delta}{2}
$$

Furthermore, the following assumptions can be made for the statistics of the sequences of $e_{q}[n]$ and $x[n][3$, pp. 754-755]:

- $x[n]$ sequence has a mean of 0 and is stationary (i.e.: the probability distribution does not change over time)

- $e_{q}[n]$ sequence is evenly distributed between $-\frac{\Delta}{2}$ and $\frac{\Delta}{2}$, i.e.: the probability distribution is $p\left(e_{q}[n]\right)=\frac{1}{\Delta}$ over the limit defined in (2.7) and 0 elsewhere

- it follows a stationary white noise sequence

- it is uncorrelated with the signal $x[n]$ 
With those assumptions, the variance of the quantization error can be shown to be $[3$, p. 755$]$ :

$$
\sigma_{e}=\frac{\Delta}{\sqrt{12}}=\frac{R / 2^{N}}{\sqrt{12}}
$$

Which can be used to relate the signal to quantization-noise ratio (SQNR) (in $\mathrm{dB})$ to the number of bits, the range, and the variance of the signal $\left(\sigma_{x}\right)$ by:

$$
\begin{aligned}
S Q N R & \equiv 20 \log _{10}\left(\frac{\sigma_{x}}{\sigma_{e}}\right) \\
& =20 \log _{10}\left(2^{N} \cdot \sqrt{12} \cdot \frac{\sigma_{x}}{R}\right) \\
& =6.02 N+10.79-20 \log _{10}\left(\frac{R}{\sigma_{x}}\right)
\end{aligned}
$$

With (2.9) the required DSP system word width can be determined based on the signal-to-noise ratio (SNR) requirements of the application.

\subsection{Digital Filters}

This section will serve as a basic introduction to DSP filters, and discuss some of the more in-depth theory for the filters implemented in this research project.

\subsubsection{Filter types}

When considering DSP filter topologies, there are two main types to choose from: Infinite impulse response (IIR) filters use feedback elements to realize transfer functions that contain terms for the current input and previous outputs. The other type, the finite impulse response (FIR) filter, consists of only feed-forward elements to realize a transfer function which only contains terms for the current and previous inputs.

IIR and FIR filters also differ considerably in their stability properties. One property of a stable system is that for an input of finite amplitude and duration, the output also has finite amplitude and duration (known as the bounded-input, boundedoutput (BIBO) stability of a system). A practical FIR filter can only operate on a finite number of samples, with finite gain for each sample, and therefore for any finite valued input the output will also be finite. On the other hand, IIR filters, due to their feedback, can be made to generate an output even after the input is removed (the output might either be oscillating or just growing in amplitude). 
Table 2.3: A summary of characteristics of the two main DSP filter topologies.

\begin{tabular}{|c|c|}
\hline \multicolumn{2}{|c|}{ DSP Filter Topologies } \\
\hline IIR & FIR \\
\hline $\begin{array}{l}\text { - } \text { stability condition varies for each } \\
\text { configuration } \\
\text { - generally lower latency } \\
\text { - not all filter responses can be im- } \\
\text { plemented practically }[5, \text { p. } 339]\end{array}$ & $\begin{array}{l}\text { - all configurations are uncondi- } \\
\text { tionally stable } \\
\text { - requires more memory } \\
\text { - requires more multipliers }\end{array}$ \\
\hline
\end{tabular}

The stability of a system could be checked by applying all possible inputs and observing the outputs, but a far less tedious method is to find the z-domain transfer function of the system and note some properties of the poles and zeros. If the poles and zeros lie within the unit circle in the z-domain then it can be shown that the system is unconditionally stable.

\subsubsection{CIC filters}

A cascaded integrator-comb (CIC) filter is a low-pass filter, often used for up-sampling or down-sampling applications where it is required to filter out the alias or image components (i.e. an interpolation or decimation filter) [7]. The CIC filter has the advantage that it requires relatively few storage elements (flip-flops) and only uses add and subtract arithmetic.

As the name implies, the filter consists of a comb stage with $N$ simple comb filters, and an integrator stage, with $N$ integrators. The comb filters subtract a delayed (by $M$ samples) version of the input signal from itself, implementing the function $y[n]=x[n]-x[n-M]$. Similarly, the integrators sum the input signal with the previous output, implementing the function $y[n]=x[n]+y[n-1]$. To illustrate, the system block diagram is shown in Figure 2.3 on the following page.

The frequency response of a CIC filter is given by:

$$
P(f)=\left[\frac{\sin \pi M f}{\sin \frac{\pi f}{R}}\right]^{2 N}
$$

As an example, the response of a CIC filter with $N=2, M=2$, and $R=10$ is plotted in Figure 2.4. 


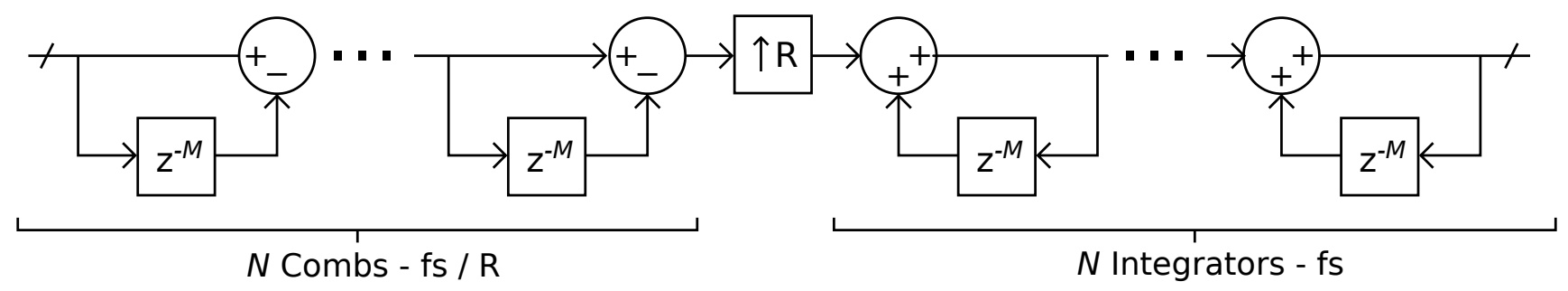

Figure 2.3: System block diagram of a CIC filter.

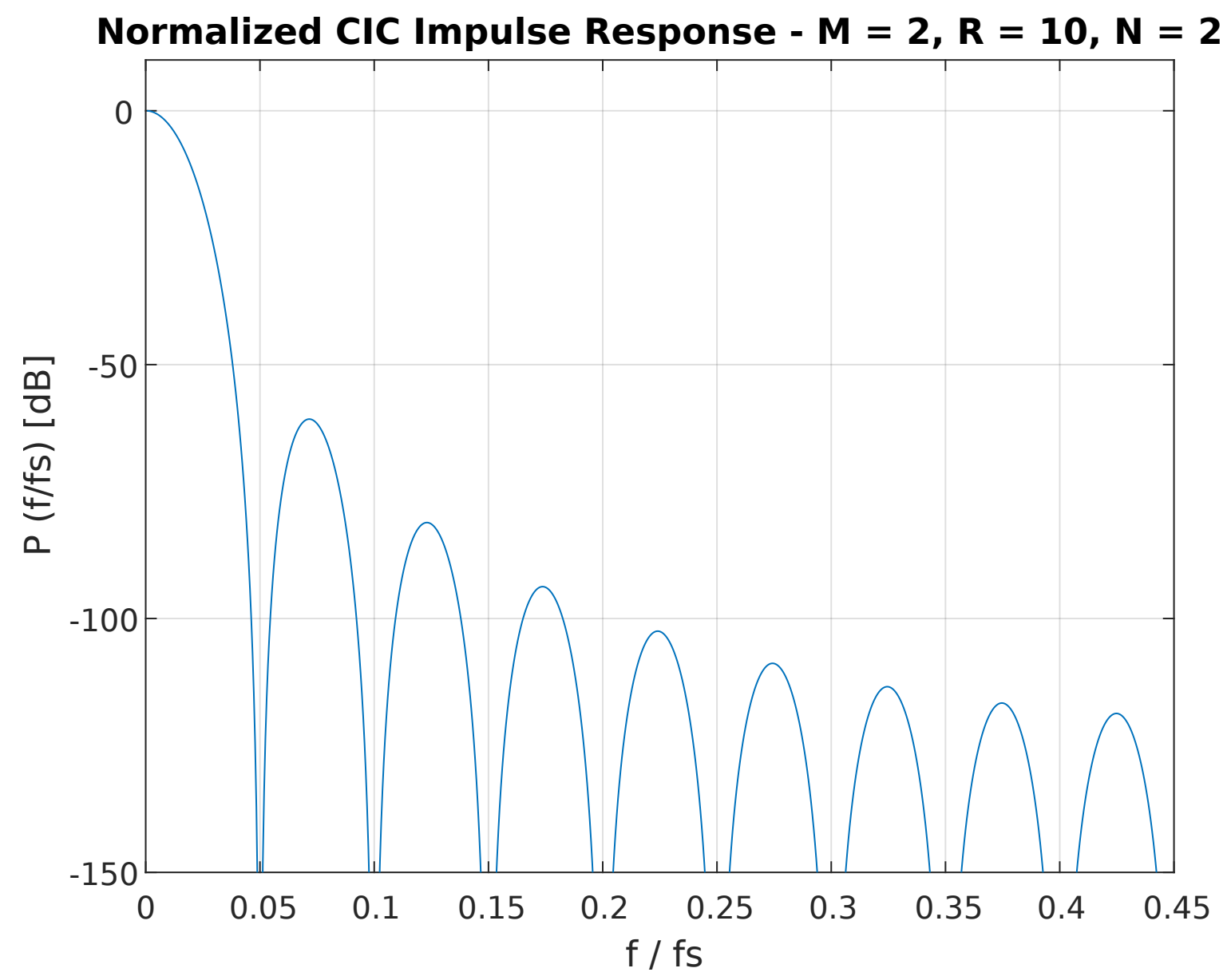

Figure 2.4: Frequency response of CIC filter. 


\subsubsection{Polyphase FIR filters}

A block diagram of a basic FIR filter is shown in Figure 2.5. The structure of the filter is simple: there is a shift register to store the current and previous input samples, the shift register is tapped off to multiply each sample by the appropriate constant (also known as a coefficient) in the impulse response, and finally each multiplied value is added together to form the current output. Therefore this structure directly implements the convolution of the input signal with the impulse response of the filter. The multiply and add operation is also known as a multiply and accumulate, abbreviated MAC. The fact that the samples are tapped off to the multipliers gives rise to the name taps to describe the number of coefficients in the impulse response.

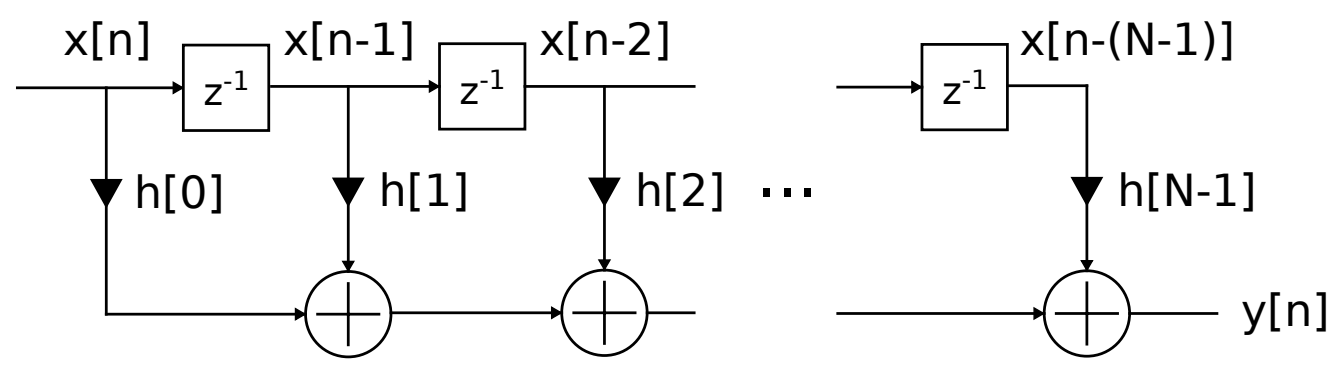

Figure 2.5: System block diagram of a FIR filter.

When a FIR filter is combined with an up-sampler at the input (say, by a factor of $L$ ), the structure can take advantage of the fact that $L-1$ of the samples are 0 , therefore the corresponding multiplier outputs will also be 0 , and they contribute nothing to the output summation. If there are $N$ filter taps, then at any given output sample only $\left\lfloor\frac{N}{L}\right\rfloor$ or $\left\lceil\frac{N}{L}\right\rceil$ MACs are active. ${ }^{4}$ By grouping the MACs according to when they are active (there are $L$ groups), it becomes clear that each group only has to operate once every $L$ samples. It can also be seen that the corresponding coefficients for each group of MACs are just a down-sampled-by- $L$ version of the overall impulse response. For each group of coefficients, the impulse response is down-sampled starting at a different sample, or phase, offset. It is the combined down-sampling while phase shifting of the impulse coefficients that leads to the name polyphase. Examples of an impulse response and its polyphase components are shown in Figure 2.6 on the following page and Figure 2.7 on page 17, respectively. Each group of MACs forms a smaller FIR filter called a polyphase bank. The banks' outputs are connected to a commutator that steps through them at the output sample rate [6; 8, Ch. 6]. This structure is shown in Figure 2.8 on page 18.

\footnotetext{
${ }^{4}$ Where $\lfloor x\rfloor$ is the floor function which rounds $x$ down to the nearest integer; similarly $\lceil x\rceil$ is the ceiling function, which rounds $x$ up to the nearest integer
} 


\section{Example FIR Impulse Response}

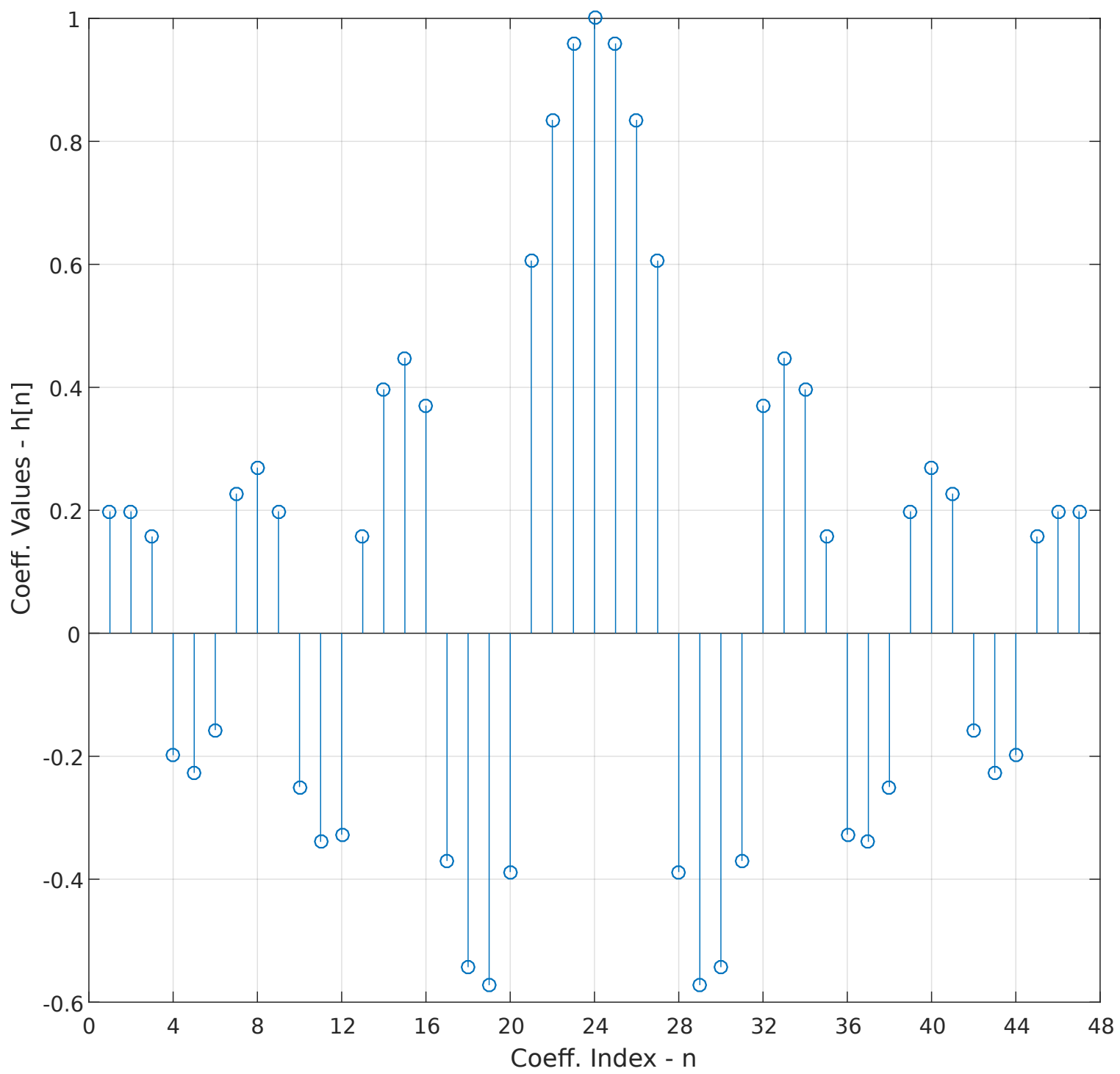

Figure 2.6: FIR filter impulse response. 


\section{Polyphase Components of FIR Impulse Response}
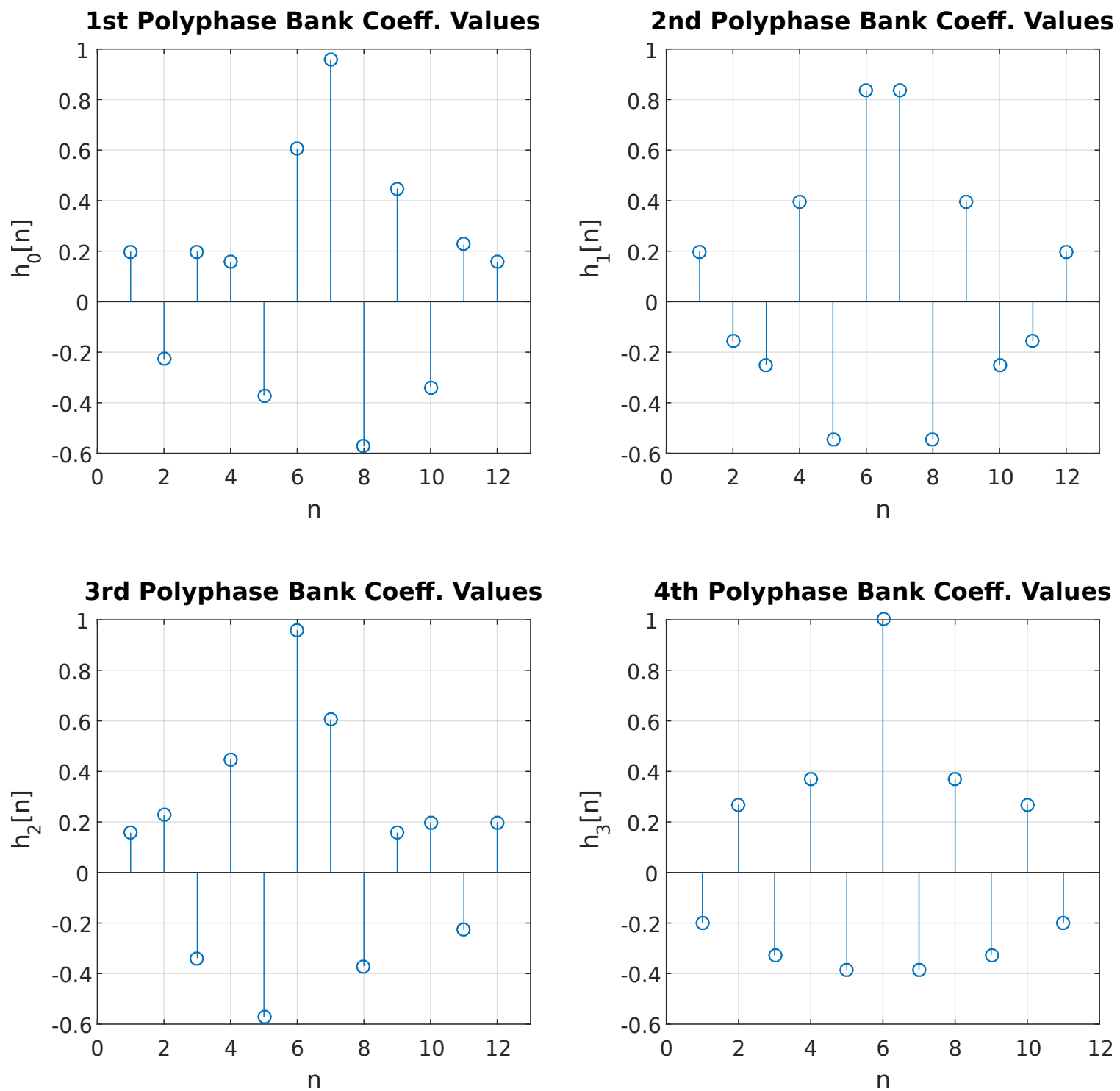

Figure 2.7: FIR filter polyphase responses. In this example, it can be seen that the 1st and 3rd set of coefficients are mirror image pairs, while the 2 nd and 4 th sets are symmetric. 


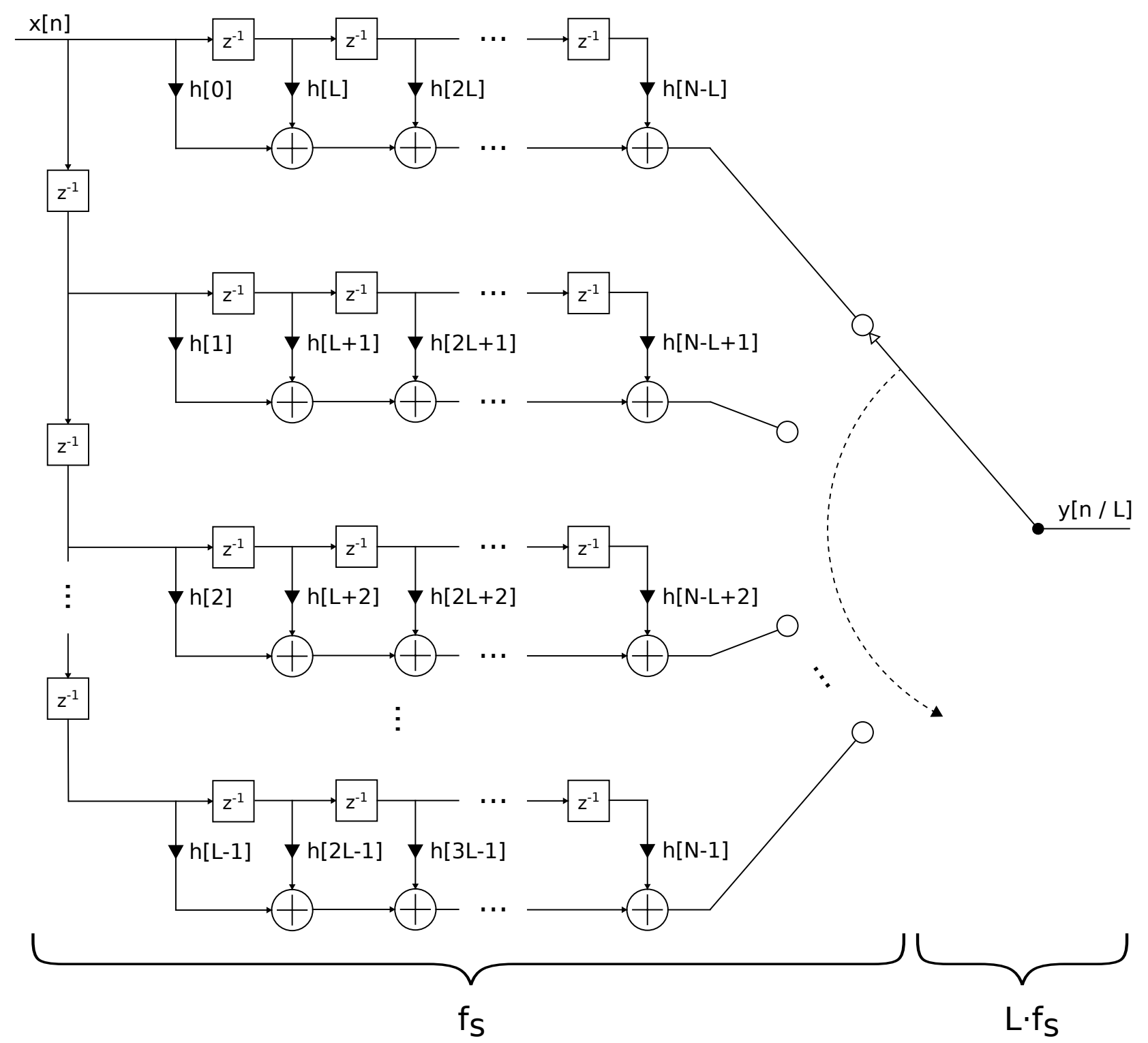

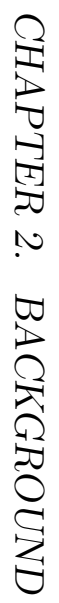

Figure 2.8: Polyphase FIR filter structure. 


\section{Coefficient Symmetry}

When the FIR filter uses a linear-phase impulse response, the filter coefficients will be symmetrical [5]. This allows half of the MACs to be eliminated, reducing the power and area requirements. Unfortunately, rearranging the FIR filter into a polyphase form complicates the problem of using a symmetrical filter, since each polyphase filter bank is not necessarily symmetric. However, careful observation of the polyphase responses reveals that each is either odd or even symmetric, or is the mirror image of one of the other polyphase responses (and vice versa). Another important observation is that the sum of a response and its mirror image is a symmetric function, and similarly the difference is also symmetric [9].

\subsection{Graph Theory}

The following section will provide some background on graph theory which will help in understanding the algorithm used for the automatic pipelining aspect of this research.

\subsubsection{Introduction}

A graph is a mathematical concept used to represent the relationship of real or abstract items. ${ }^{5}$ Each item forms a vertex of the graph, and the association between items forms an edge on the graph. For example, a graph could be used to model the transportation routes between major cities, while abstracting the exact geography; each city is a vertex and the highways are the edges. Similarly, graphs can be used to represent the flow of signals between logic gates in a digital circuit, where each gate is a vertex and the nets from gate outputs to gate inputs are the edges. For example, the gate-level diagram of a half-adder circuit is shown in Figure 2.9a on the next page, and its representation as a graph is shown in Figure 2.9b.

There are two types of edges in a graph, directed and undirected. Directed edges have a defined direction; they start on a given vertex and end on another. An undirected edge, in contrast, has no defined direction; it simply shows that two vertices are connected but not that one follows from the other.

If a graph contains only undirected edges, then it is also called undirected. A graph with any number of directed edges (even if it also has undirected edges) is a

\footnotetext{
${ }^{5} \mathrm{Graph}$ in this context should not be confused with what is also known as a chart or a plot, which shows how a set of independent and dependent values are related.
} 


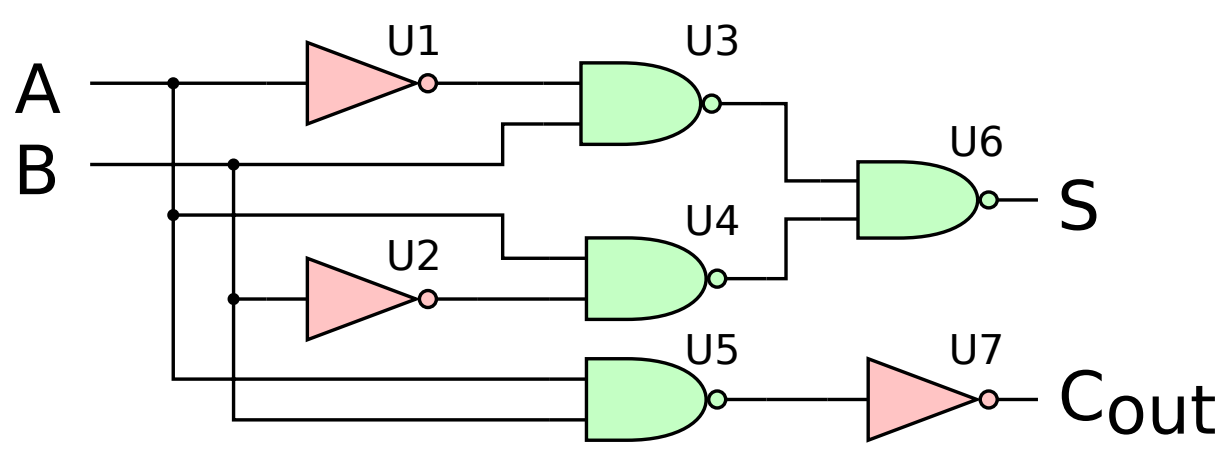

(a) Gate-level logic diagram for a half adder circuit.

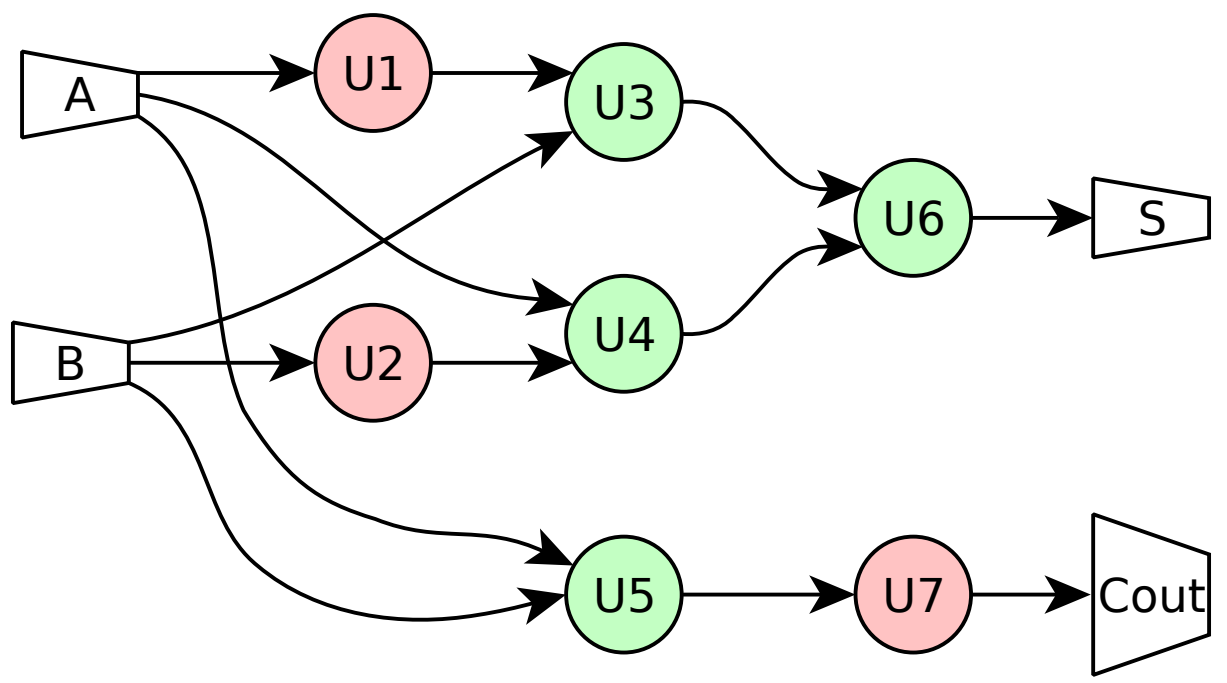

(b) Equivalent graph for a half adder circuit.

Figure 2.9: A half adder circuit and its graph equivalent. 
directed graph. If there are any undirected edges in a directed graph, they can be considered equivalent to a pair of directed edges with opposite direction.

The transpose of a directed graph, $G_{T}=\operatorname{transpose}(G)$, is the graph which has all the same vertices as $G$, but with the direction of all edges reversed. For example, if an edge in $G$ is directed from vertex $v_{1}$ to $v_{2}$, i.e. $e_{G}: v_{1} \rightarrow v_{2}$, then the corresponding edge in $G_{T}$ would be directed from $v_{2}$ to $v_{1}$, i.e.: $e_{G T}: v_{2} \rightarrow v_{1}$.

A vertex in a directed graph can be classified a parent if there are any edges directed away from it. Similarly, if a vertex has any edges directed toward it, then the vertex is considered a child. Observe in Figure 2.10 that a vertex can be simultaneously a parent and a child if it has edges directed both toward and away from it.

If a vertex has no parents and only children, then it is further classified as a source. Similarly, any vertex that has only parents and no children is a sink.

A cycle in a directed graph is a set of edges that when followed will create a path leading from a vertex back to itself. If a directed graph has no cycles, then it is a directed acyclic graph $(D A G)$.

\subsubsection{Graph traversal}

Graph algorithms involve the task of visiting all of the vertices and edges in a certain sequence. The usefulness of the algorithms come from the actions that are taken as each vertex or edge is visited [10, p. 302].

A graph's nodes can be traversed in a number of different ways, but two principal algorithms are the depth-first search and the breadth-first search.

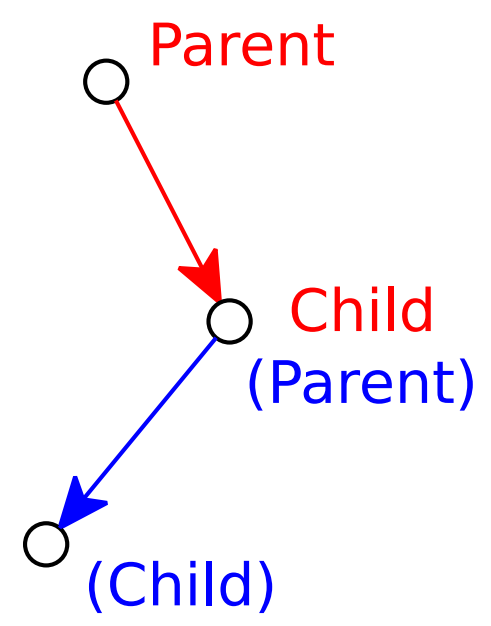

Figure 2.10: Example showing how a vertex can be both a child (for the red, nonbracketed relationship), or a parent (in the case with blue and brackets). 


\section{Depth-first search}

In a depth-first search, the algorithm starts at a single vertex or a small set of vertices (usually source vertices) which might also be referred to as the root or roots. The current vertex is flagged as having been "visited". Then, for each child vertex that has not been visited yet, the depth-first search is called recursively on that child vertex $[10$, p. 302]. Following these steps, it can be seen that the algorithm follows every edge as deep as is possible, hence the name depth-first search.

\section{Breadth-first search}

The breadth-first search is the complement to the depth-first search. The algorithm also begins with the root(s), and flags them as visited as it iterates through them. For each vertex, it visits all of the children that have not been visited yet. Every vertex that is visited also gets flagged as now being visited. It then repeats for each unvisited child of the children (grandchildren) of the vertex, and so on, until there are no more unvisited vertices [10, p. 307]. In other words, the algorithm steps through all the vertices that are 1 edge away from the root, then all of the vertices that are 2 edges away, then 3 edges away, etc. Hence it traverses the whole "breadth" of the graph in stages, before proceeding to more vertices.

\section{$2.4 \quad$ RF Transmitters}

In the following section, the basic system-level concepts of RF transmitters will be covered. This is intended to provide background for the parts of the thesis that detail the application of the research to wireless communication systems.

\subsubsection{Quadrature amplitude modulation}

A common method for modulating digital information onto a carrier sinusoid is quadrature amplitude modulation (QAM). The method for QAM is to simultaneously, but independently, amplitude-modulate two sinusoids which have the same frequency as the carrier, but which are phase shifted by $90^{\circ}$ to each other. One of the two sinusoids is known as the in-phase (I) component, the other is the quadrature $(Q)$ component. The final modulated signal is formed by adding the in-phase and quadrature components together.

The number of amplitude levels for each phase component is determined by the number of bits to be transmitted in each symbol. Plotting each phase component on 
a phasor diagram, it becomes obvious that QAM is equivalent to modulating both the amplitude and phase of the carrier sinusoid. The phasor plot, showing each discrete amplitude of the I and Q components shows a constellation of points, which is why the plot is more commonly known as a constellation diagram or just constellation. An example constellation with 4 levels for I and Q is shown in Figure 2.11; since there are 16 constellation points, this encoding is called 16-QAM.

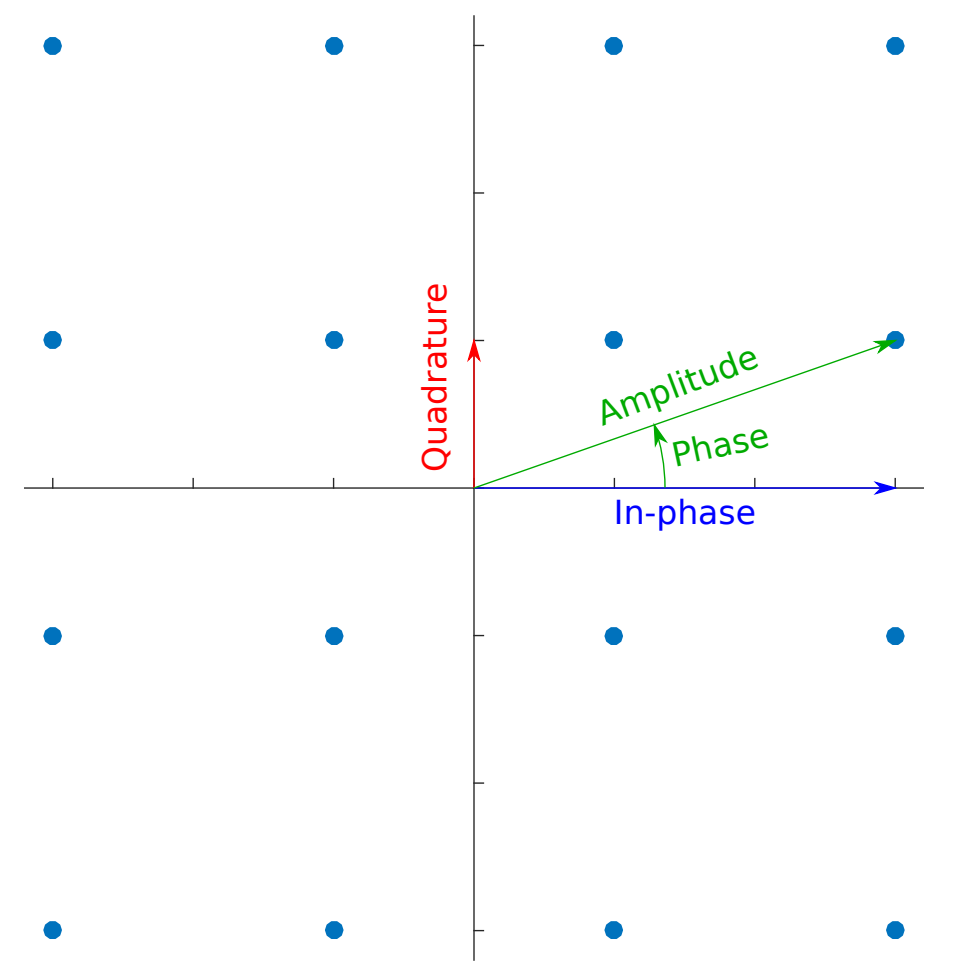

Figure 2.11: Example of a 16-QAM constellation, showing how the I and Q components add together to make a new phasor representing a sinusoid of the same frequency but with a different amplitude and phase shift.

\subsubsection{Error Vector Magnitude}

The transmitted constellation points may be distorted from their ideal positions due to a variety of reasons, such as circuit non-linearities [11, Sec. 5.12], or quantization noise in the baseband digital representation. A measure of the deviation of the transmitted constellation points from their ideal location is the error vector magnitude (EVM). The difference in the transmitted point $\left(t_{i}\right)$ and the ideal point $\left(a_{i}\right)$ forms a vector called the error vector $\left(e_{i}=t_{i}-a_{i}\right)$ (see Figure 2.12 on the following page for an illustration). Then the EVM for a given symbol is defined as the ratio of the magnitudes of the error vector to the ideal point $\left(\mathrm{EVM}_{i}=e_{i} / a_{i}\right)$. EVM is more 
commonly calculated as an rms value for a large number of symbols to obtain a statistical average [12, Eq. 2.23]:

$$
\mathrm{EVM}_{\mathrm{rms}}=\sqrt{\frac{\frac{1}{N} \sum_{i=1}^{N}\left|e_{i}\right|^{2}}{\frac{1}{N} \sum_{i=1}^{N}\left|a_{i}\right|^{2}}}
$$

The rms EVM is often specified and reported as a percentage by multiplying the value produced from (2.10) by $100 \%$.

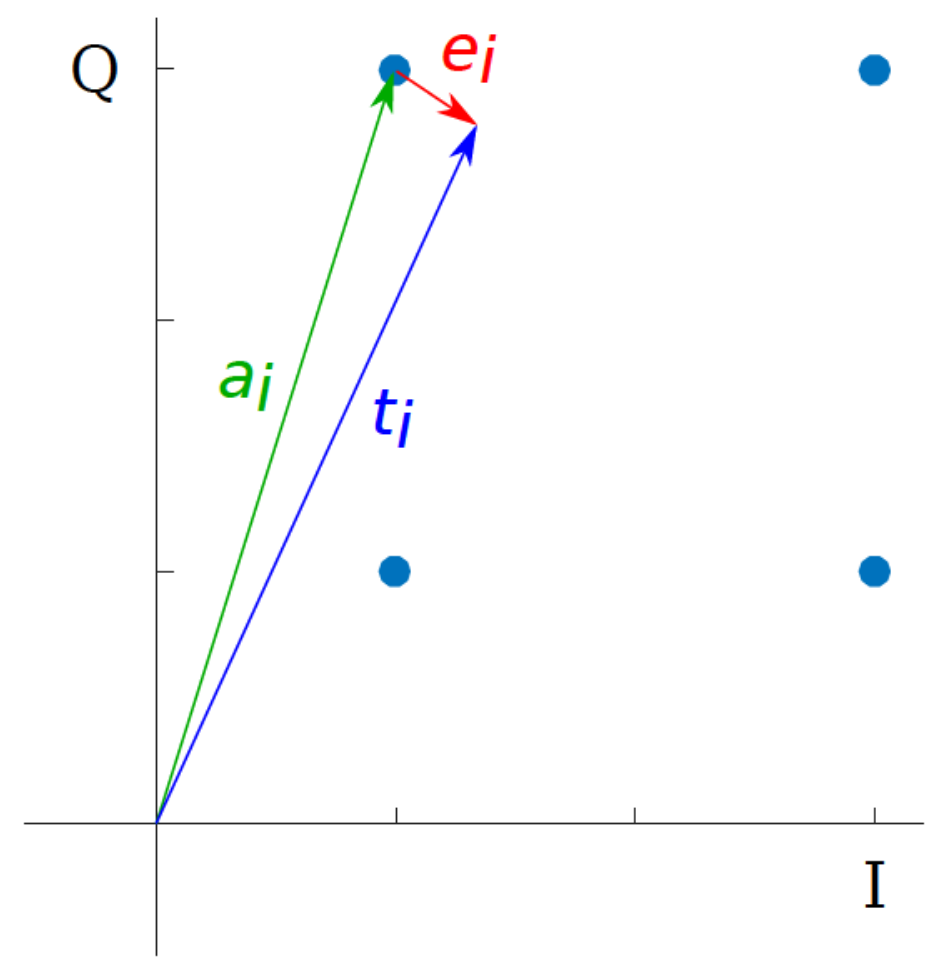

Figure 2.12: Illustration of the various vectors used in the calculation of EVM.

\subsubsection{Superheterodyne}

A widely used transmitter architecture is the superheterodyne (superhet) transmitter [11, Sec. $4.2 ; 13]$. The basic principle is to translate the baseband (BB) waveform first to a frequency band centred around some fixed intermediate frequency $(I F)$, and then translate the IF up to a tunable RF band. The frequency translation at each stage is performed by multiplying the signal with a sinusoid from a synthesizer; the multiplication circuit is more commonly called a mixer. There are also some filters 
required along the signal path due to aliasing at the output of the DAC and after the mixers ${ }^{6}$ [11, pp. 131-132]. A superhet receiver operates in the reverse order of the transmitter, with the signal moving from RF to IF, then to BB. A block diagram for a standard superhet transmitter is shown in Figure 2.13.

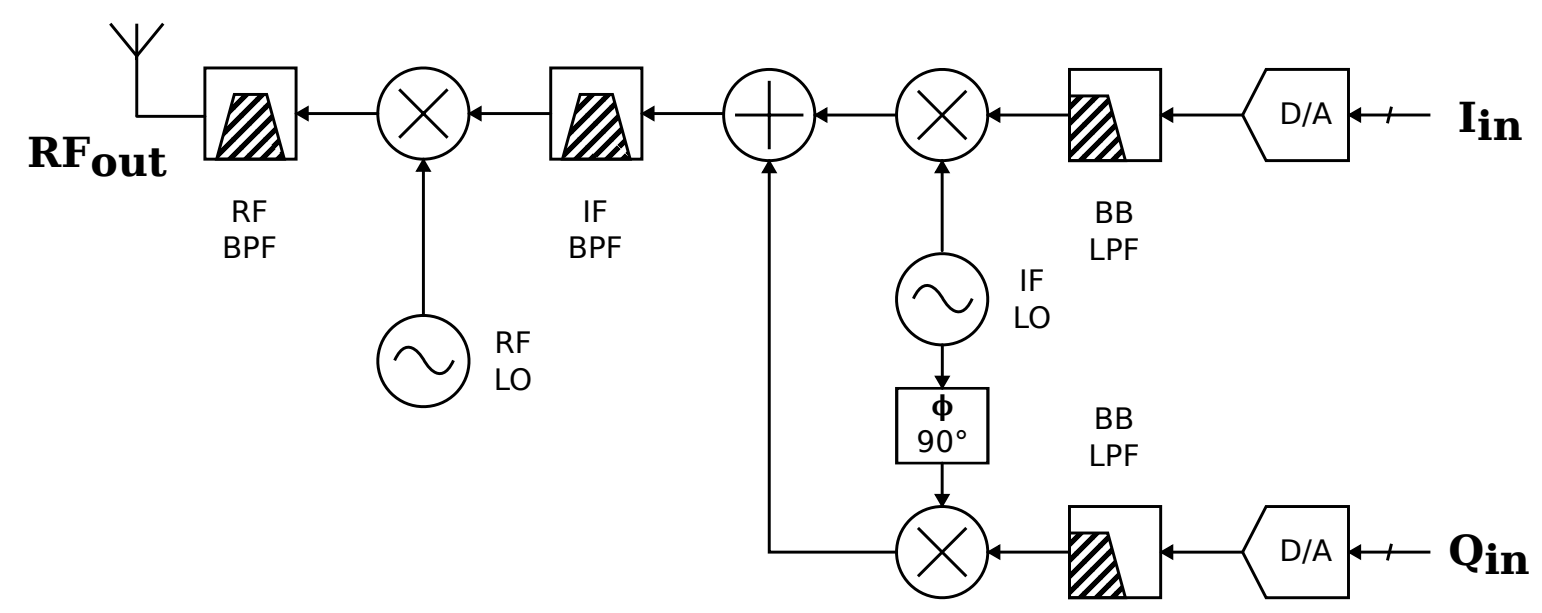

Figure 2.13: Example of a superheterodyne transmitter. For simplicity the various amplifiers in the signal paths are not shown.

\subsection{Clocked CMOS}

This section will provide an overview of the Clocked CMOS logic family.

\subsubsection{Introduction}

Clocked CMOS ( $\left.\mathrm{C}^{2} \mathrm{MOS}\right)$ is a modification to standard CMOS that involves adding clock transistors in series with the standard CMOS pull-up (PMOS) and pull-down (NMOS) networks, respectively [1]. When one of the clock transistors is between the positive rail and the PMOS network, it is called a header; the corresponding transistor that is between the negative rail and the NMOS network is known as the footer. As seen in Figure 2.14a on the next page, the footer transistor for the NMOS network is driven by a clock signal, while the header for the PMOS is connected to an inverted version of the clock. The clock transistors can also be placed at the output node of the gate, as shown in Figure 2.14b on the following page.

\footnotetext{
${ }^{6}$ The aliasing at the output of the mixer can be deduced by using the trigonometric identity for the product of sinusoids: $\cos \left(\omega_{C}\right) \cdot \cos \left(\omega_{B B}\right)=\frac{1}{2}\left[\cos \left(\omega_{C}+\omega_{B B}\right)+\cos \left(\omega_{C}-\omega_{B B}\right)\right]$; or by convolving the frequency domains, similar to the analysis for sampling done in Section 2.1.1 on page 4 .
} 


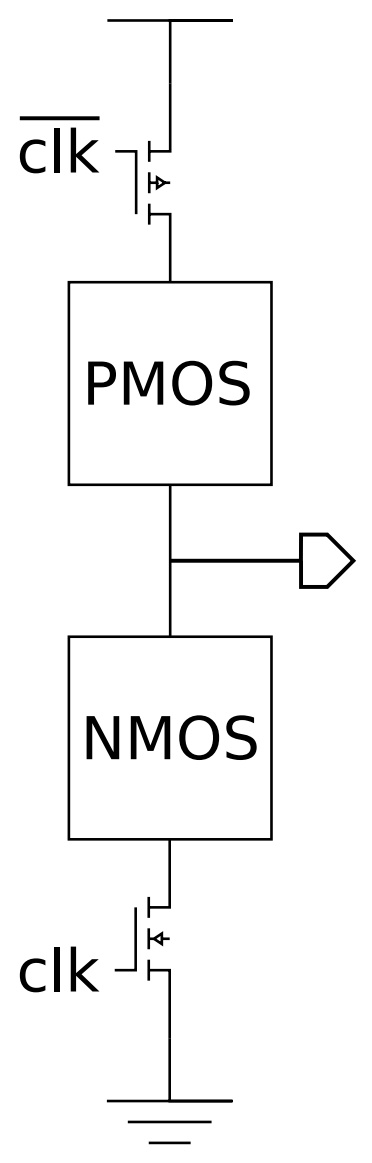

(a) using header and footer transistors

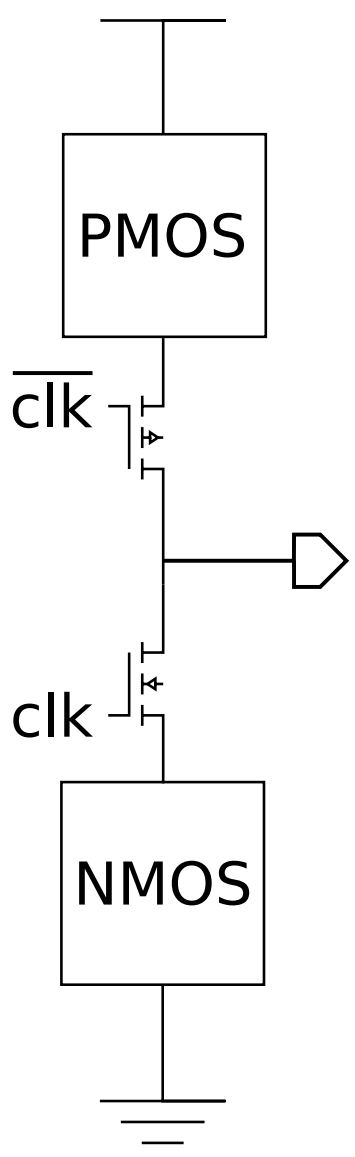

(b) using transistors at the output node

Figure 2.14: Schematics of the two $\mathrm{C}^{2} \mathrm{MOS}$ gate styles. 
When the clock is high, the $\mathrm{C}^{2} \mathrm{MOS}$ gate behaves as a normal CMOS gate, and is said to be transparent; however, when the clock is low, if the input(s) to the gate change, the output is not affected, and the gate is opaque. Thus a $\mathrm{C}^{2} \mathrm{MOS}$ gate is equivalent to a combination of a CMOS gate and a D-type latch.

Two back-to-back D latches, clocked by complementary clock signals, form a Dtype flip-flop (also called a master-slave flip-flop) [14, pp. 17-19]. Similarly, by combining two $\mathrm{C}^{2} \mathrm{MOS}$ gates back-to-back (with the clock connections swapped on the second gate), a combination flip-flop (FF) and logic gate is created.

There are advantages to placing the clock transistors at the supply rails, such as:

- to minimize leakage current (both subthreshold leakage and junction diffusion leakage) [15], similar to power gating [14, Sec. 5.3.2];

- or to potentially simplify routing and clock distribution by merging clock transistors.

However, there is at least one important disadvantage which must be considered, which is charge sharing, as explained in the following sub-section.

\subsubsection{Charge Sharing}

For a $\mathrm{C}^{2} \mathrm{MOS}$ gate with header and footer transistors, charge sharing occurs in the following scenario.

1. The input is stable when the clock signal goes low, shutting off the header and footer, leaving the gate in the opaque state. In this state, the output node is floating and the output capacitance, $\mathrm{C}_{\text {out }}$ is either charged to the $\mathrm{V}_{\mathrm{DD}}$ potential or discharged to the $\mathrm{V}_{\mathrm{SS}}$ potential.

2. If the input changes, some of the transistors in the PMOS and NMOS networks may conduct, thus connecting $\mathrm{C}_{\text {out }}$ to some diffusion capacitance, $\mathrm{C}_{\mathrm{diff},\{\mathrm{P}, \mathrm{N}\}}$ and the charge is now shared and redistributed among them. The voltage at the output will change according to the capacitive voltage divider equation. Either:

(a) $\mathrm{C}_{\text {out }}$ was charged to $\mathrm{V}_{\mathrm{DD}}$ and the charge sharing to any $\mathrm{C}_{\text {diff,N }}$ will reduce the output voltage; or,

(b) $\mathrm{C}_{\text {out }}$ was discharged and some charge from a $\mathrm{C}_{\text {diff,P }}$ will be shared, thus raising the output voltage. 
The change in the output voltage is called charge sharing noise [14, p. 393]. Refer to Figure 2.15 for a visualization of the charge sharing process. While charge sharing is not desirable, it can be tolerated, so long as the charge sharing noise is not greater than the threshold voltage of the transistors in the succeeding gate [14, Ch. 9].

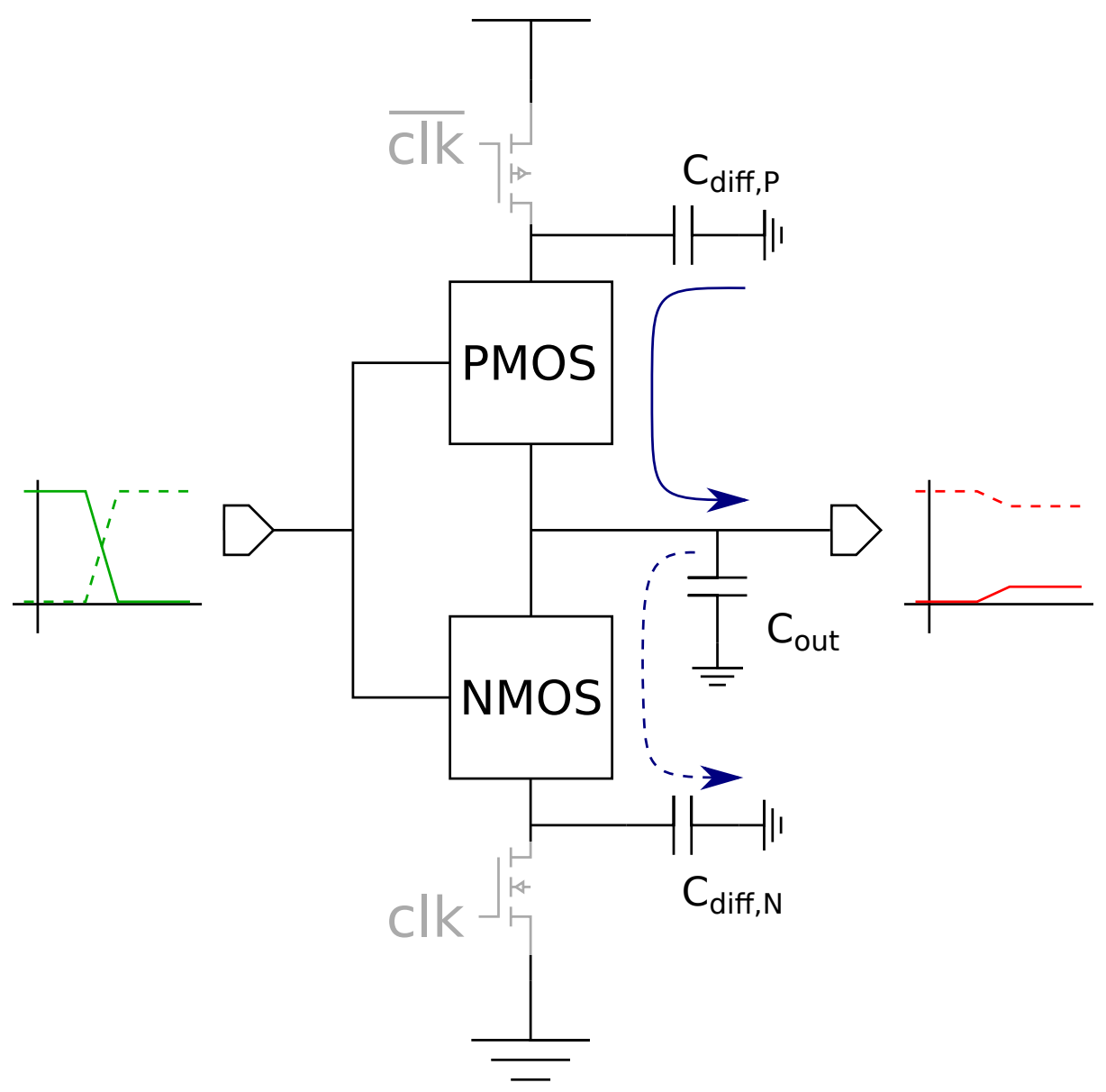

Figure 2.15: Illustration of the charge sharing problem. While the clock transistors are off, the input changes, causing the charge to redistribute between $\mathrm{C}_{\text {out }}$ and $\mathrm{C}_{\mathrm{diff},\{\mathrm{P}, \mathrm{N}\}}$.

\subsection{Pipelining}

Pipelining is a technique used to increase the data speed of a digital circuit. Typically, a digital circuit block is partitioned such that there are FFs that form a register of the block inputs; the output of this register is connected to the structure of combinational logic gates that make up the logical function of the block (often called a cloud of logic); and the outputs of the cloud are connected to the inputs of another set of FFs that 
form the output register. This structure can be seen in Figure 2.16a on the following page.

Assuming that there is no clock skew, the maximum clock speed (or minimum clock period, $\left.T_{c l k, m i n}\right)$ that the registers can be run at is determined by the delay through the input register FFs $\left(t_{p c q}\right)$, the propagation time through the logic cloud $\left(t_{p d}\right)$, and the setup time of the output register FFs $\left(t_{\text {setup }}\right)$ [14, Eq. 10.1]:

$$
T_{c l k, m i n} \geq t_{p c q}+t_{p d}+t_{s e t u p}
$$

From (2.11), it can be seen that the clock period can be reduced by reducing any of the delays; however, since reducing the FF delays would require redesigning the FFs, the easiest change can be reduction of the logic delay. The logic delay is easily reduced by decreasing the depth of the logic cloud by partitioning it into smaller clouds and placing additional FFs at the intermediate points, as illustrated in Figure 2.16b. Each smaller logic cloud coupled with a FF is called a pipeline stage. The number of stages placed between input and output is referred to as the pipeline depth.

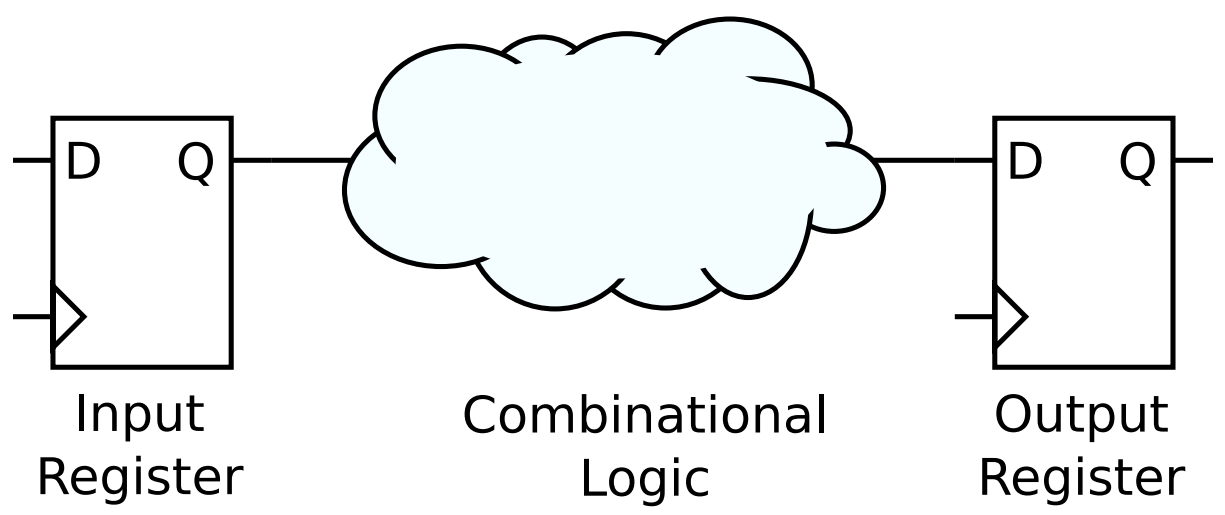

(a) A typical logic block with input and output registers.

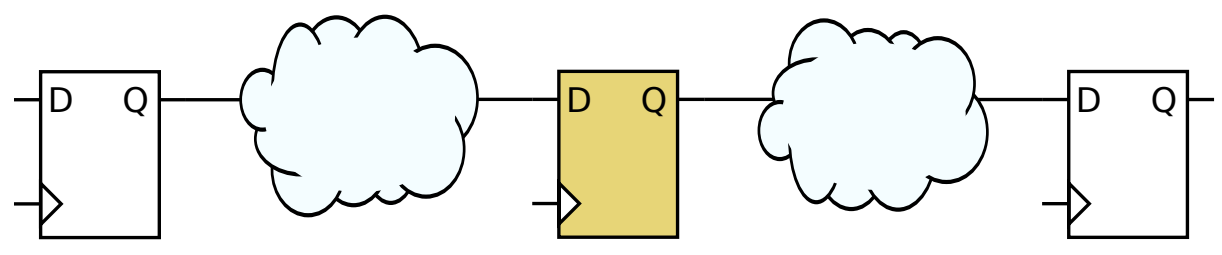

(b) Pipelining the logic block by inserting FFs between the existing registers.

Figure 2.16: Schematics illustrating the use of pipelining.

Thus, by reducing the clock period, pipelining increases the throughput of the circuit (i.e. the number of values that can be loaded, or unloaded, in a given time), 
but due to the increased depth of stages, it may also increase the latency of the circuit (i.e. the time it takes from a value being loaded at the input to its corresponding result being unloaded at the output) [16, p. 117; 17, Sec. 7.5].

\subsection{Summary}

This chapter covered the background of much of the theory involved in this research topic. Topics included the fundamentals of DSP and digital filters, the basics of graph theory, a brief discussion of RF transmitters, an overview of $\mathrm{C}^{2} \mathrm{MOS}$, and finally the concept of pipelining for digital circuits. The next chapter will detail the design process of this research, which relies on the concepts covered in this chapter. 


\section{Chapter 3}

\section{Design}

This chapter presents the design steps for both the QAM transmitter implemented on an FPGA, and the CIC filter implemented on an IC using a $130 \mathrm{~nm}$ CMOS process. The IC was fabricated through the Canadian Microelectronics Corporation's (CMC's) multi-project wafer service [18], which is subsidized based on a peer review of the proposed design.

\subsection{Automatic Pipelining}

This section will detail the design and implementation of the automatic pipelining algorithm.

\subsubsection{Algorithms}

As mentioned in section 2.6, pipelining involves adding FFs in between some clouds of logic. The minimum period that the clock can be set to is determined by the longest propagation delay through any of the pipeline stages. For example, if there are two pipeline stages, one with a logic delay of $t_{p d, 1}$ and the second stage has delay $t_{p d, 2}$; then supposing $t_{p d, 1}>t_{p d, 2}$, then the minimum clock period must meet $T_{c l k, \text { min }} \geq t_{p c q}+t_{p d, 1}+t_{\text {setup }}$, i.e. the minimum period is determined by the maximum delay. In general, if there are $n$ stages, with delays $t_{p d, 1}, t_{p d, 2}, \ldots, t_{p d, n}$, then the minimum clock period must be:

$$
T_{c l k, \text { min }} \geq t_{p c q}+\max \left(t_{p d, 1}, t_{p d, 2}, \ldots, t_{p d, n}\right)+t_{\text {setup }}
$$

If some of the delay of the slower stages were transferred to the faster stages, then the shortest minimum period could be achieved; i.e. optimally, the delays of all stages 
will be equal. This could be achieved with careful placement of the pipeline registers.

One method for optimally placing the pipeline registers is called retiming. It involves redistributing the registers to minimize the delay, but guarantees the new circuit to be functionally equivalent to the old [19].

The procedure for pipelining via retiming is as follows [20, pp. 16-17; 21, p. 6]:

1. Decide how many stages of pipelining should be used, i.e. the depth.

2. Add a number of series FFs for the desired depth, either to the input or output of the circuit. These are called the retiming FFs.

3. Run retiming on this new circuit. The algorithm attempts to distribute the FFs around the circuit to minimize the clock period.

There are two possible drawbacks to retiming, however. Firstly, step 2 generally requires the designer to manually edit the netlist to add the FFs. The second, and usually more critical, drawback is that since any of the FFs may end up on almost any path, it complicates the development of design-for-test (DFT) methodologies, such as automatic test pattern generation and checking [22].

Instead, two algorithms were developed based on some prior work of, and in collaboration with, Mr. Yatish Kumar; one adds pipeline FFs and ensures delay balancing, the other just ensures delay balancing for use on the $\mathrm{C}^{2} \mathrm{MOS}$ circuitry (which doesn't need FFs since the logic is inherently clocked). These two algorithms will be detailed in the following sections.

\section{Automatic pipelining for FPGA}

To understand the desire for pipelining on an FPGA, it is worthwhile to examine the general structure of an FPGA. Generally, the basic building block of an FPGA is known as a slice ${ }^{1}[24$, Ch. 5] which consists of a combination of a lookup-table (LUT) (for the combinational logic function) with a bypassable $\mathrm{FF}$ on the output, as can be seen in Figure 3.1 on the following page. The LUT is typically implemented as a 1- or 2-bit wide static random access memory (SRAM) whose address lines are connected to the function inputs, and output are the data lines; the contents of the SRAM is programmed to match the truth table of any arbitrary function. The FPGA is formed from a large array of these slices (with modern implementations including some fixed function logic blocks, such as dedicated CPUs and digital signal processors). From

\footnotetext{
${ }^{1}$ This is Xilinx's terminology; Altera uses the term adaptive logic module (ALM) [23, pp. 2-5-2-6].
} 
examining the slice's structure it can be concluded that since all the FFs are already placed on the IC, there is no reason not to take advantage of them for maximum speed performance by applying pipelining (this, of course, is not true if power is more of a concern).

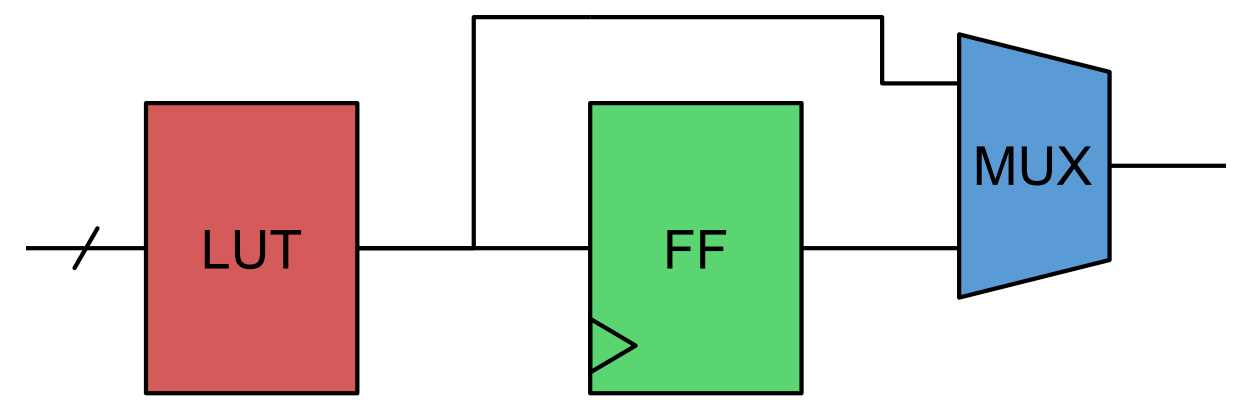

Figure 3.1: Illustration of an FPGA slice.

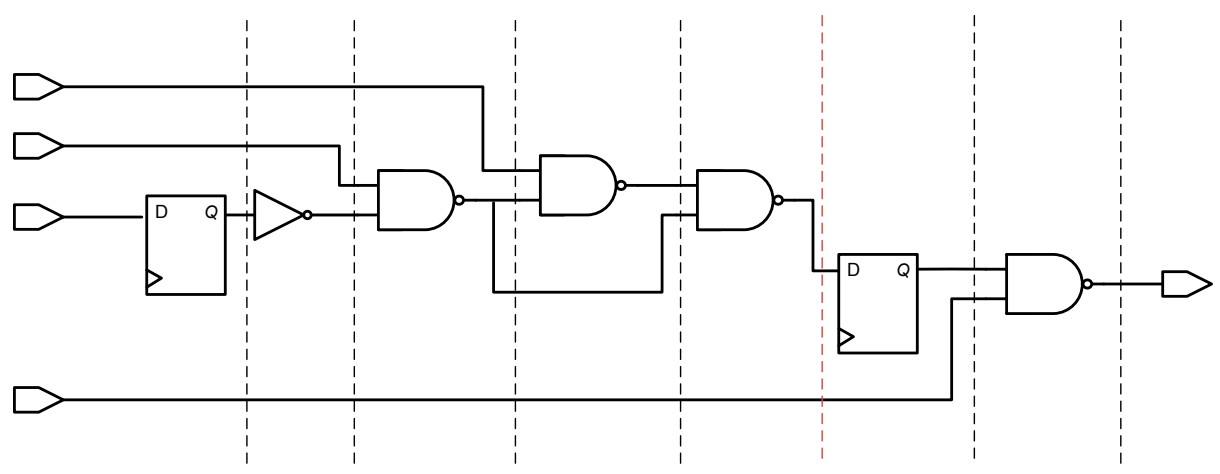

(a) An example of a logic block before pipelining.

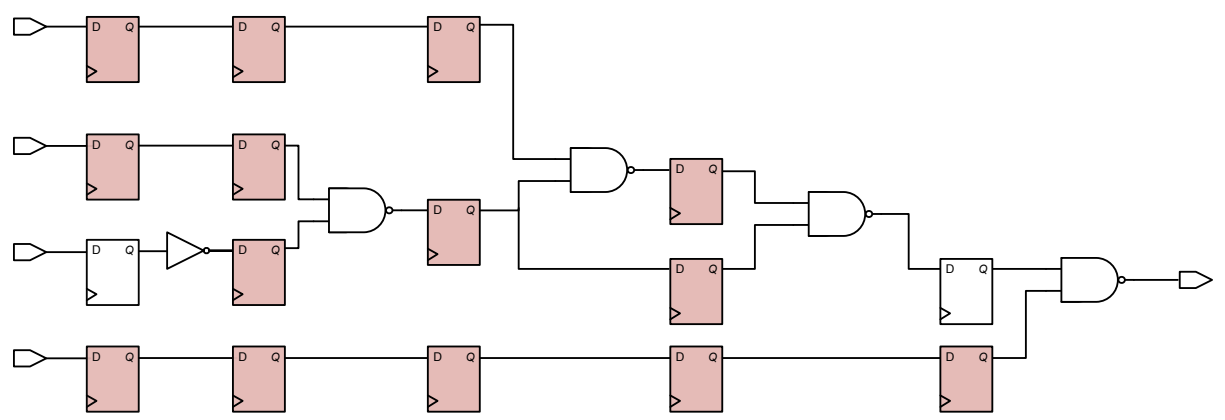

(b) Pipelining the logic block by inserting FFs at the output of each gate, thus creating a maximally deep pipelined circuit.

Figure 3.2: An example circuit and its pipelined version.

To implement pipelining for the FPGA, pipeline FFs needed to be added into the Verilog netlist at appropriate stages in the logic clouds. By adding the FFs to the netlist, they would get mapped to the FPGA FF in the slice. 
The algorithm that was developed as part of this thesis adds pipelining FFs to the circuit such that there is a certain number of logic gates between each stage. The action of the algorithm also guarantees that all register depths are balanced.

1. Read in the Verilog netlist.

2. Create a graph $\left(G_{C}\right)$ based on the netlist structure. Each vertex represents a logic gate and each edge is a wire connecting the output of one gate to the input of another.

3. Process the graph by traversing it and labelling each vertex with its depth in the hierarchy ${ }^{2}$. The graph is traversed via a depth-first search (DFS) ${ }^{3}$ on the transposed graph of the logic, $G_{C, T}$, which has its root connected to the output(s) of the netlist instead of the input(s). The transpose is taken so that the output(s) are considered to be the last event(s) that need to occur.

4. Insert FFs between the output of any gate and the inputs of its children. The number of FFs inserted is calculated by the difference in the gates' depth, divided by the desired number of stages between pipeline FFs. In some instances, no FF is inserted.

The pseudocode for the algorithm is shown in Figure 3.3 on the next page.

\section{Delay balancing for $\mathrm{C}^{2} \mathrm{MOS}$}

For the CIC filter that was implemented with $\mathrm{C}^{2} \mathrm{MOS}$, a slightly different algorithm than the previous one was developed. The need for a different algorithm arises because the $\mathrm{C}^{2} \mathrm{MOS}$ logic is inherently pipelined and therefore new FFs do not need to be added: only $\mathrm{C}^{2} \mathrm{MOS}$ buffers need to be added to balance delays amongst the different paths. For example, if the initial circuit is the one shown in Figure 3.4a on page 36, then the buffers need to be added in the locations indicated in Figure 3.4b.

The delay balancing algorithm that was developed operates in a similar manner to the pipelining algorithm, the only difference is the action performed by the algorithm for inserting buffers. Broadly speaking, the algorithm operates by first generating a list of a certain type of path called a bypass path. A bypass path is one which originates from the same source as a string of gates, and runs parallel along the string, converging back at a common point, such as a gate, or at the output ports

\footnotetext{
${ }^{2}$ This is similar to, but not the same as a topological sort [10, pp. 304-307], since a topological sort forces an order onto otherwise parallel units [10, p. 304].

${ }^{3}$ The idea for using a DFS to traverse the graph was proposed by Mr. Yatish Kumar.
} 
procedure PIPELINE(graph $G$ )

$r \leftarrow \operatorname{root}(G)$

$G_{T} \leftarrow \operatorname{transpose}(G)$

$r_{T} \leftarrow \operatorname{root}\left(G_{T}\right)$

$\triangleright$ get the root of the transposed graph

$\operatorname{DFS}\left(G_{T}, r_{T}\right.$, labelDepth $)$

$\triangleright$ do depth-first search on $G_{T}$

$\operatorname{DFS}(G, r$, insertFFs $)$

end procedure

procedure DFS(graph $G$, vertex $v$, procedure postVertex) $\triangleright$ conceptual DFS

mark $v$ as seen

for all unseen neighbours of $v$ called $n$ do

$\operatorname{DFS}(G, n)$

end for

$\operatorname{post\operatorname {Vertex}}(v)$

end procedure

procedure LABELDEPTH(vertex $v$ )

$v_{\text {depth }} \leftarrow 0 \quad \triangleright$ Used to keep track of what the depth of $v$ should be.

for all children of $v$ do

$c \leftarrow$ current child of $v$

$c_{\text {depth }} \leftarrow \operatorname{getDepth}(c)$

if $c_{\text {depth }}>v_{\text {depth }}$ then

$v_{\text {depth }} \leftarrow c_{\text {depth }}$

end if

end for

$\operatorname{setDepth}\left(v, v_{\text {depth }}+1\right) \quad \triangleright$ depth of $v$ is 1 greater than max. of its children

end procedure

procedure INSERTFFS(vertex $v$ )

if $v$ is not root then

for all children of $v$ (sorted by depth) do

$c \leftarrow$ current child of $v$

add the correct number of FFs between $v$ and $c$

end for

end if

end procedure

Figure 3.3: Automatic pipelining and delay balancing algorithm. The DFS algorithm shown is reproduced from [10]. It is only conceptual, however, since the actual implementation in the Perl Graph module is based on a state-machine and hashes [10, p. 289], which is more efficient computationally. The setDepth and getDepth procedures are trivial, and not shown. 
(i.e. all outputs are considered common in the context of the algorithm). An example of a bypass path can be seen in Figure 3.4a. Then, for each gate, for each of its output paths that are of the bypass type, a number of buffers, equal to the maximum depth of any parallel paths, are added. The details of the algorithm are shown in Figure 3.5, however those parts that were the same as the previous algorithm are not shown.

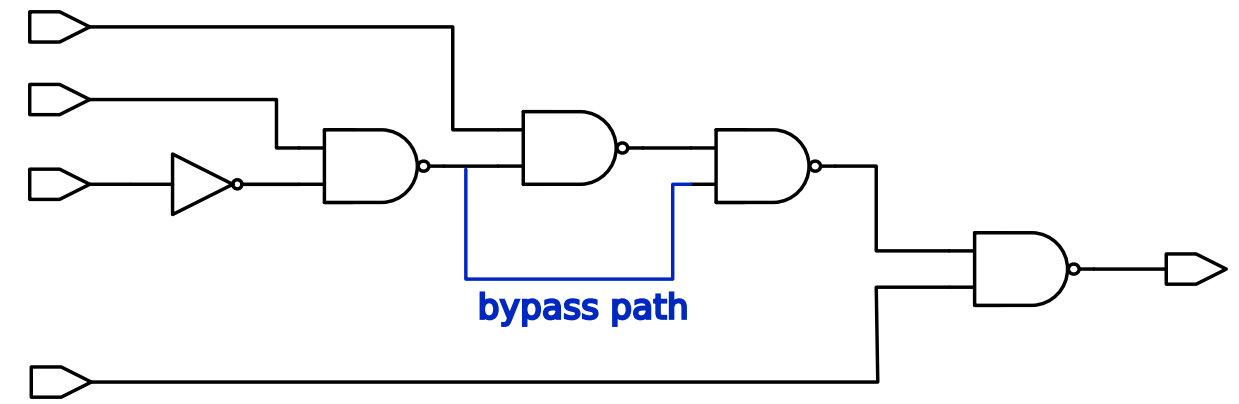

(a) An example of a logic block before delay balancing. Also shown is an example of a bypass path.

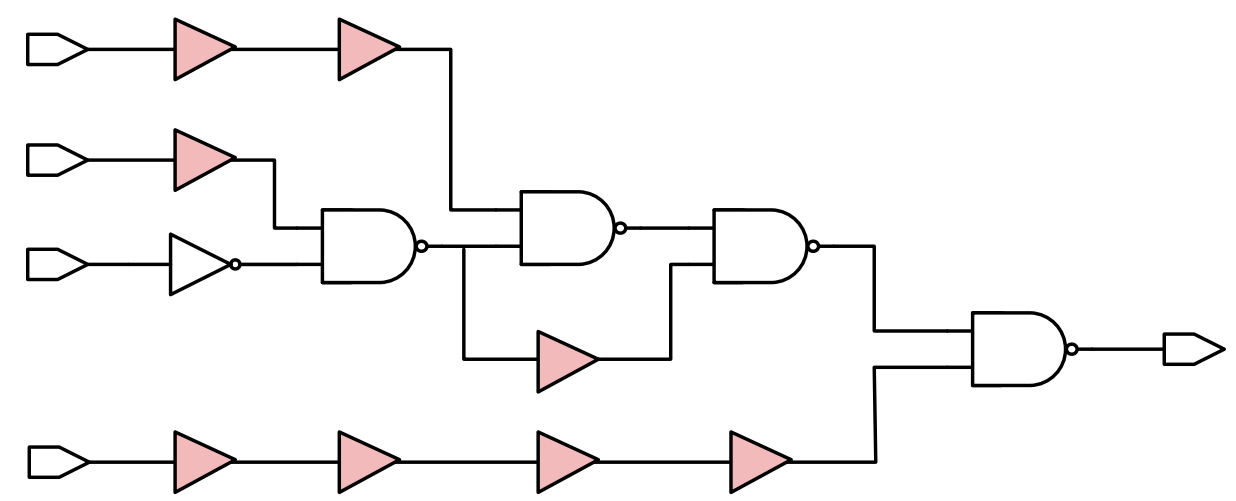

(b) Inserting buffers (shown in red shading) at appropriate points to achieve delay balancing.

Figure 3.4: An example circuit and its delay balanced version.

\subsubsection{Perl Script}

Perl was chosen to implement the above algorithm for a few reasons:

- It is a well established programming language with a large support network, e.g. the Comprehensive Perl Archive Network (CPAN) [25].

- One of its primary uses is for processing large text files.

- And most importantly, a module ${ }^{4}$ is available for reading in, and writing out,

${ }^{4} \mathrm{~A}$ Perl module is a collection of related functions (c.f. library for other programming languages). 


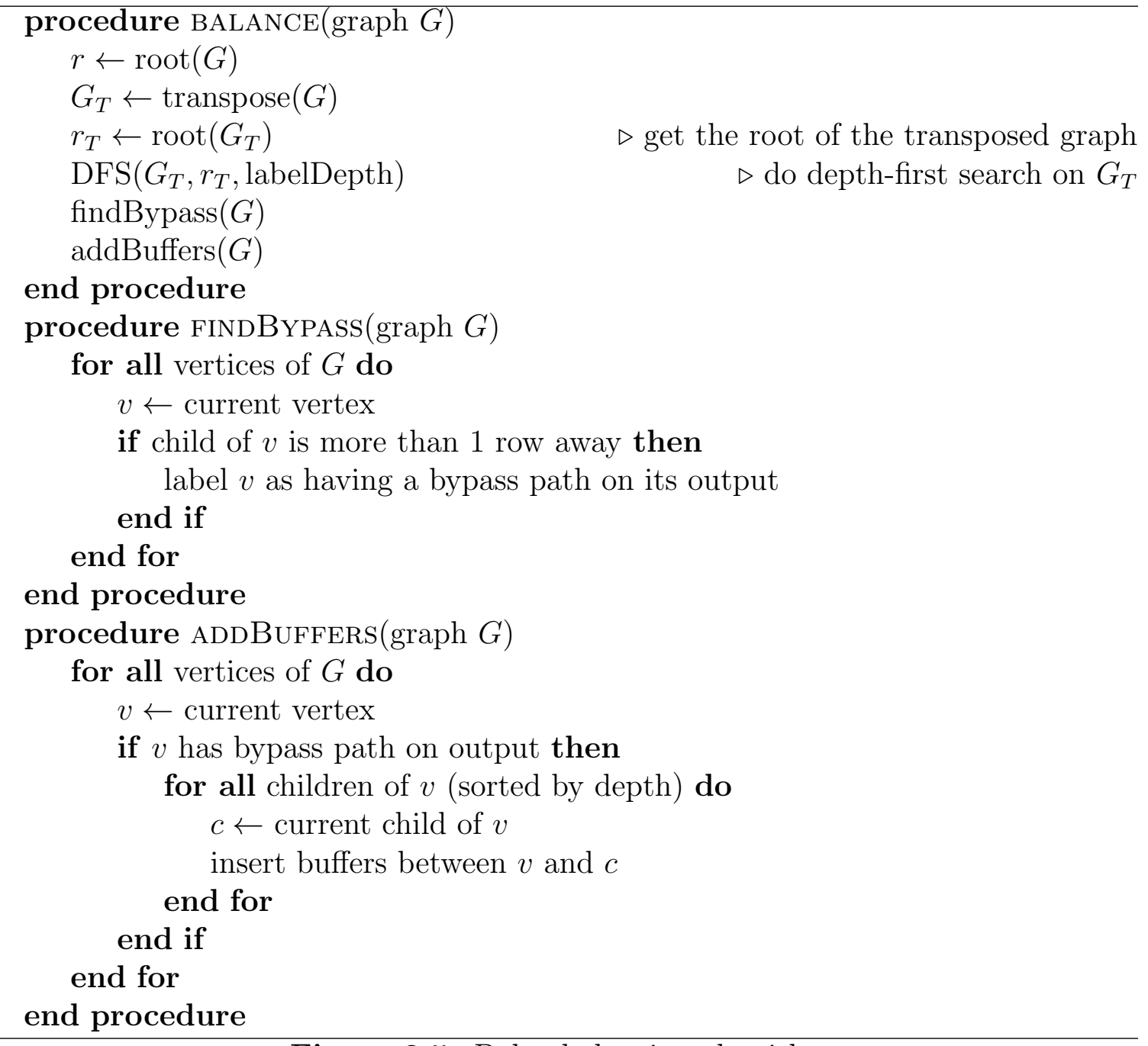

Figure 3.5: Delay balancing algorithm. 
Verilog netlists, Verilog-Perl [26]; and also a relatively efficient graph processing module, Graph [10] [27] is available ${ }^{5}$.

The Verilog-Perl module represents all the Verilog modules read in as an objectoriented data structure [28]. This makes it easier to manipulate the netlist using the appropriate methods.

The Graph module provides many of the most widely used graph algorithms, implemented relatively efficiently, and provides a convenient call-back mechanism to perform an action for each node processed. The post-vertex callback mechanism, for example, was used in the part of the algorithm that labels each vertex with its hierarchical depth.

\subsection{Integrated Circuit Cells}

This section details the design of the basic integrated circuit blocks, such as the input/output (I/O) pad buffers, and the $\mathrm{C}^{2} \mathrm{MOS}$ standard cells.

Before going into any detail, it should be mentioned that since PMOS transistors have lower mobility than NMOS, most CMOS cells are designed such that the PMOS widths are some ratio of NMOS widths. For the IBM $130 \mathrm{~nm}$ technology, it was found that the PMOS transistor in a CMOS inverter should be three times as wide as the NMOS transistor to achieve equal rise and fall times. Therefore all of the CMOS cells designed were based on this 3-to-1 ratio for PMOS widths to NMOS widths. The other parameters of the IBM $130 \mathrm{~nm}$ technology are summarized in Table 3.1.

Table 3.1: Summary of the IBM $130 \mathrm{~nm}$ kit parameters.

\begin{tabular}{cc}
\hline Parameter & Value \\
\hline Advertised gate length & $130 \mathrm{~nm}$ \\
$V_{D D}$ & $1.2 \mathrm{~V}$ \\
Layers of metal & 8 \\
Minimum transistor width & $160 \mathrm{~nm}$ \\
\hline
\end{tabular}

\footnotetext{
${ }^{5}$ Thanks to Mr. Yatish Kumar for finding these modules.
} 


\subsubsection{Input/Output Buffers}

In order to detect signals off-chip at data rates of a few Gbps, high-speed output buffers were designed.

\section{Inverter chain buffers}

The first type of output buffer designed was based on a popular technique of using a string of inverters where each has an increasing width compared to the last [29]. An output buffer was developed based on the technique of using an even number of inverters of increasing width. The theoretical optimum scaling value is Euler's constant $(e)$, so this was used as a starting value; but after simulation a scaling value of 2.5 was found to work better. It was also found that using 9 stages gave the best result, and an extra stage of same size was added at the input to provide an even number of inversions (ensuring logic equivalence). The schematic for this output buffer is shown in Figure 3.6.

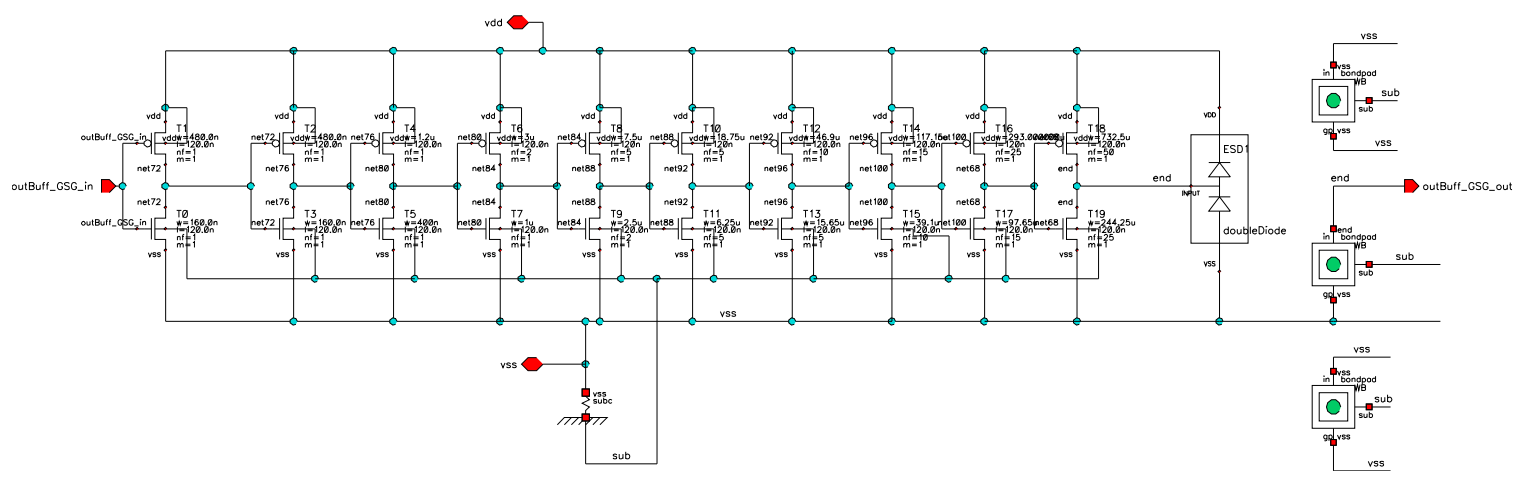

Figure 3.6: Schematic of the output buffer based on an inverter chain.

These buffers and pads, as well as a simple input buffer consisting of a single inverter, were then translated to a layout in the IBM $130 \mathrm{~nm}$ process. A chip size of 1 by $1 \mathrm{~mm}$ was awarded from CMC. This layout is shown in Figure 3.7 on the next page.

\section{CML style buffers}

In testing the inverter chain buffers, it was discovered they did not operate adequately beyond 2 Gbps. Therefore, a new high-speed buffer cell was developed based on the CML style buffers covered in [30].

The output buffer cell was designed to drive an off-chip load of $50 \Omega$. This value for the load arose since the chip was to be packaged, placed on a test-jig PCB, with 


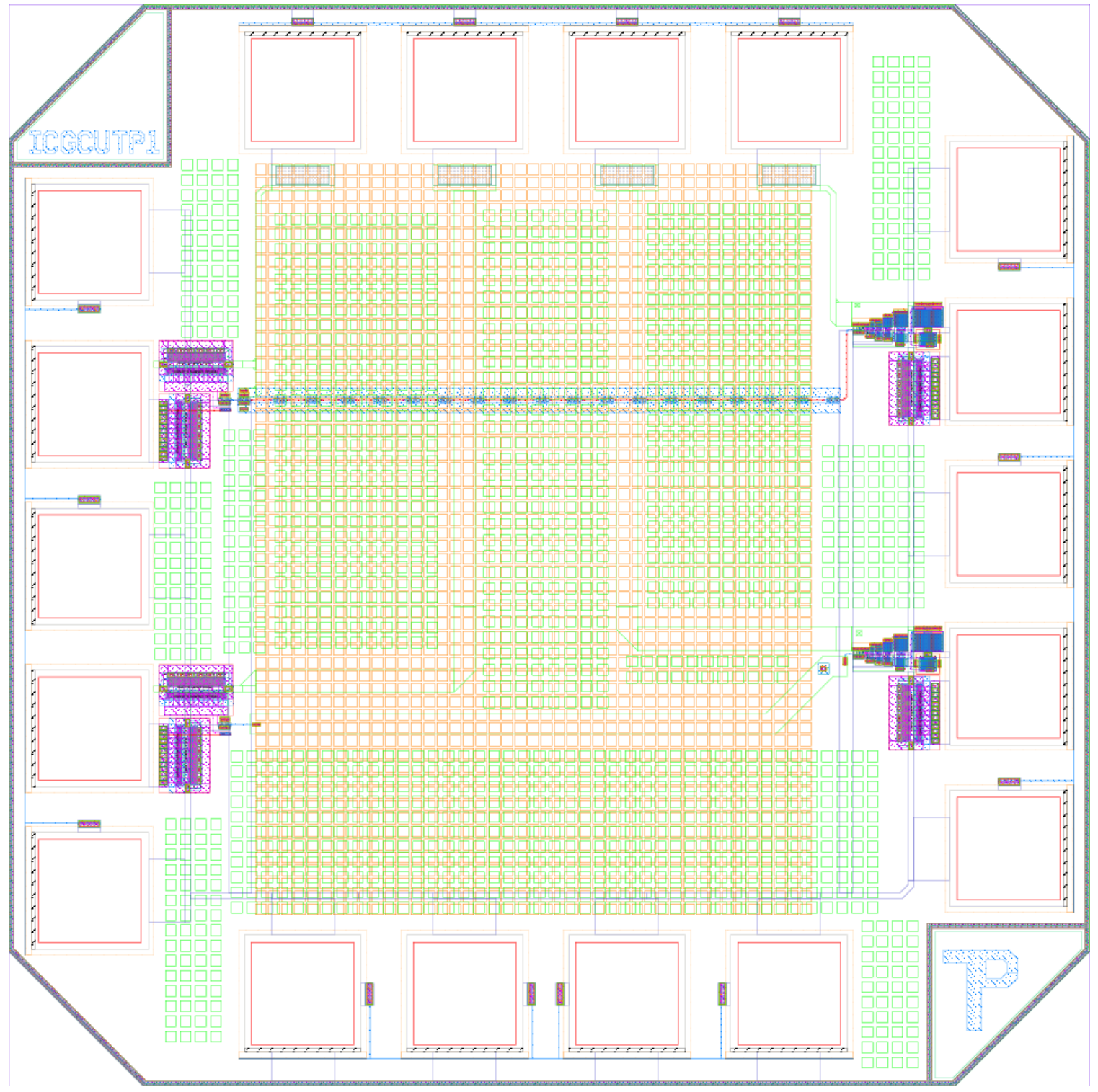

Figure 3.7: Layout of the buffers test chip. 
the signal lines connected to $50 \Omega$ SMA connectors, which would allow the signals to be attached to test equipment through $50 \Omega$ SMA cables. The output swing per differential side was desired to be $0.6 \mathrm{~V}$; therefore, the required tail current for the CML output buffer would be [30, Eq. 12.7]:

$$
I_{\text {tail }}=\frac{2 \cdot 0.6 \mathrm{~V}}{50 \Omega}=24 \mathrm{~mA}
$$

The optimum current density of the NMOS transistors for peak operating frequency is considered to be around $0.4 \mathrm{~mA} \mathrm{um}^{-1}$ in the $130 \mathrm{~nm}$ process. This leads to a value for the width of the transistors of $W_{N M O S}=24 \mathrm{~mA} / 0.4 \mathrm{~mA} \mathrm{\mu m}^{-1}=60 \mu \mathrm{m}$.

Since the CML transistors are fairly big, an inverter chain buffer was designed to drive the CML transistor's gates. The width scaling was two, and there were seven stages. The last stage was used to drive one transistor in the CML differential pair, while the second last inverter was used to drive the other transistor in the pair.

The schematic for the CML output buffer is shown in Figure 3.8, and its corresponding layout is in Figure 3.9 on the next page. The on-chip resistance loads for the CML differential pair are also set to $50 \Omega$ to match the off-chip load impedance. In layout, the resistors are placed in a common-centroid configuration to improve matching.
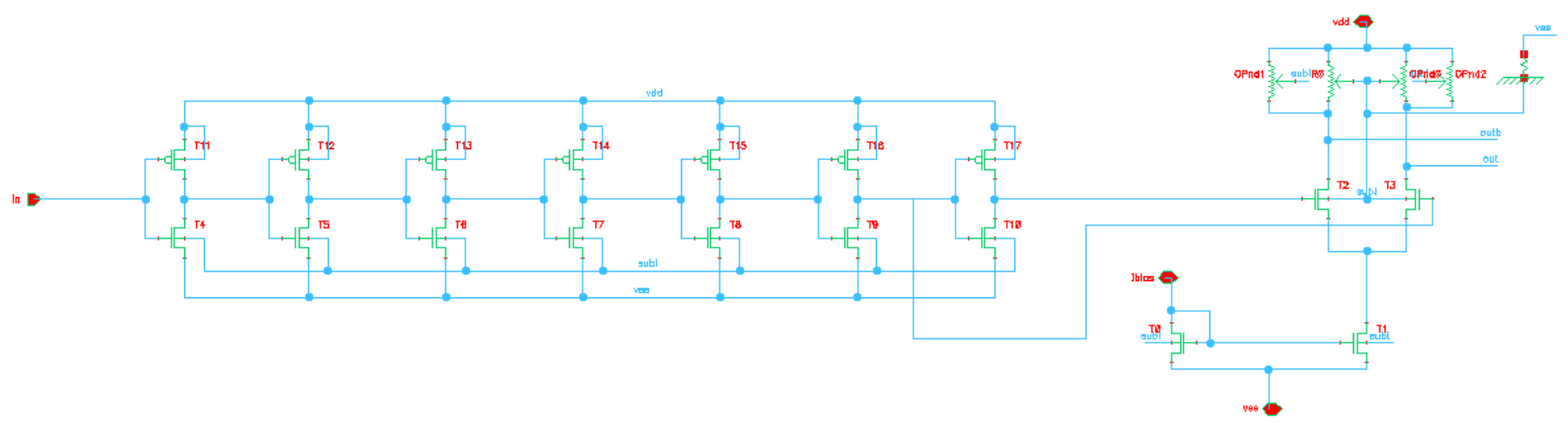

Figure 3.8: Schematic for the CML output buffer.

In a similar method to the CML output buffer, an input buffer for the highspeed logic clock was designed. In order to generate non-overlapping clocks for the $\mathrm{C}^{2} \mathrm{MOS}$ latches, the positive and negative outputs of the CML buffer were designed to drive LO-skew inverters. A LO-skew inverter is a CMOS inverter with a PMOS transistor which is narrower than in a typical CMOS inverter, which results in the output switching at a lower input threshold. To drive the clock buffers, the LOskew inverters were set to an NMOS and PMOS width of $5.13 \mu \mathrm{m}$. The impedance 


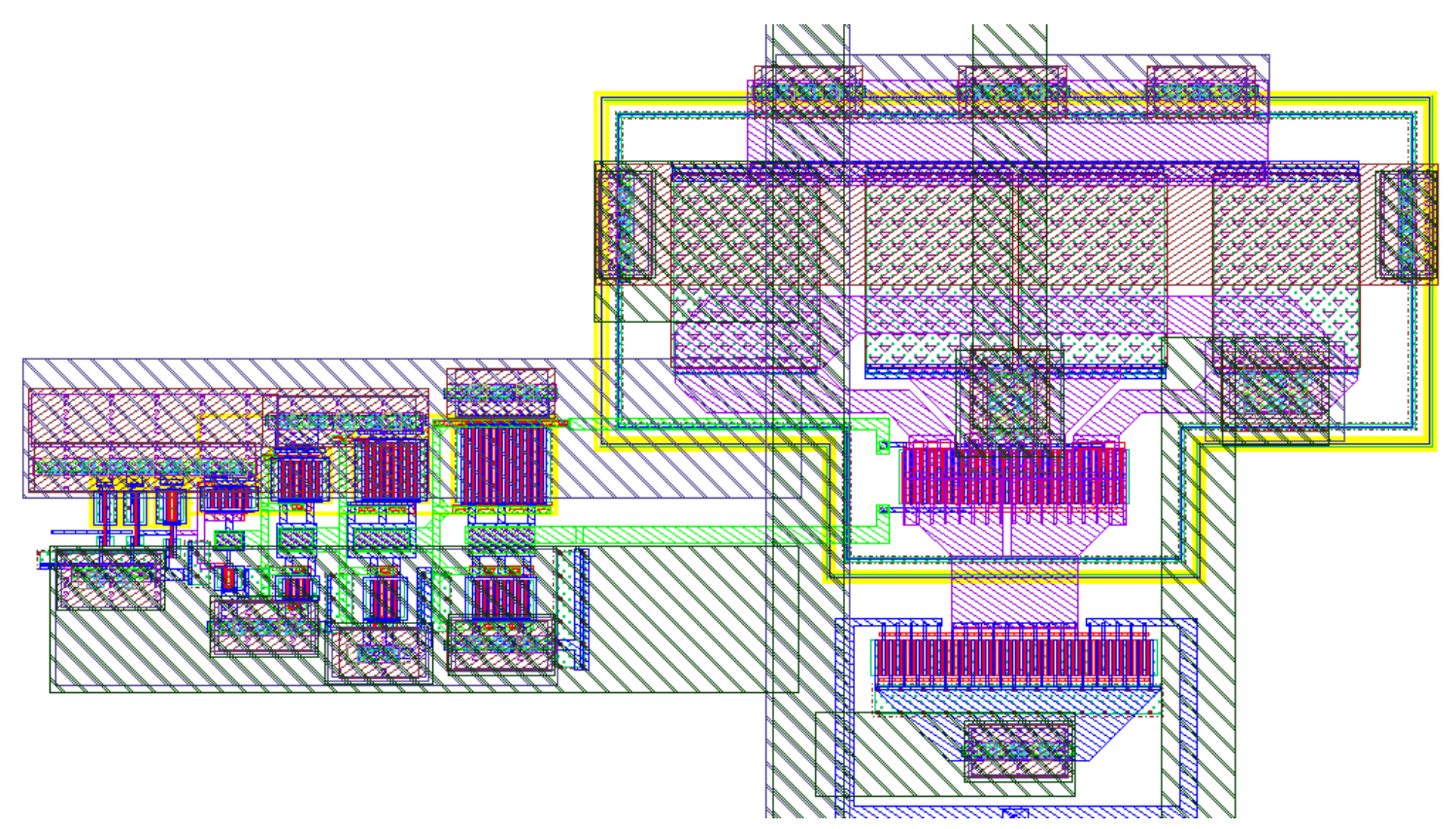

Figure 3.9: Layout for the CML output buffer.

of the input capacitance of these inverters is roughly equivalent to a $1 \mathrm{k} \Omega$ load on the CML buffer. For a full $1.2 \mathrm{~V}$ swing from the CML buffer, this requires a tail current of $2.4 \mathrm{~mA}$, which translates to NMOS transistor widths of $6 \mu \mathrm{m}$. Ultimately, after running some simulations, the transistor widths were reduced to $3 \mu \mathrm{m}$ for better performance.

The schematic for the input buffer is shown in Figure 3.10 on the following page, and the corresponding layout is in Figure 3.11 on page 44. Due to the geometry of the layout resistors, it was decided to break them up into two parts along their length, then arrange them in a common-centroid pattern.

\subsubsection{C $\mathrm{C}^{2} \mathrm{MOS}$ Standard Cells}

A small library of standard cells was developed for implementing the $\mathrm{C}^{2} \mathrm{MOS}$ logic. The cells created were: a buffer, an inverter, a 2-input NAND, and a 2-input NOR. The transistors were sized so that the gates' worst case rise and fall times were the same as a static CMOS inverter. This is simply achieved by scaling the width by the worst-case number of series transistors between the output and either the $V_{D D}$ rail (for PMOS) or the $V_{S S}$ rail (for NMOS).

If the minimum width of a transistor is $W_{\text {min }}$, then in a static CMOS inverter the NMOS width is kept to the minimum $\left(W_{N M O S}=W_{m i n}\right)$; and, as previously mentiond, for the $130 \mathrm{~nm}$ technology, the PMOS width is set to three times as much 


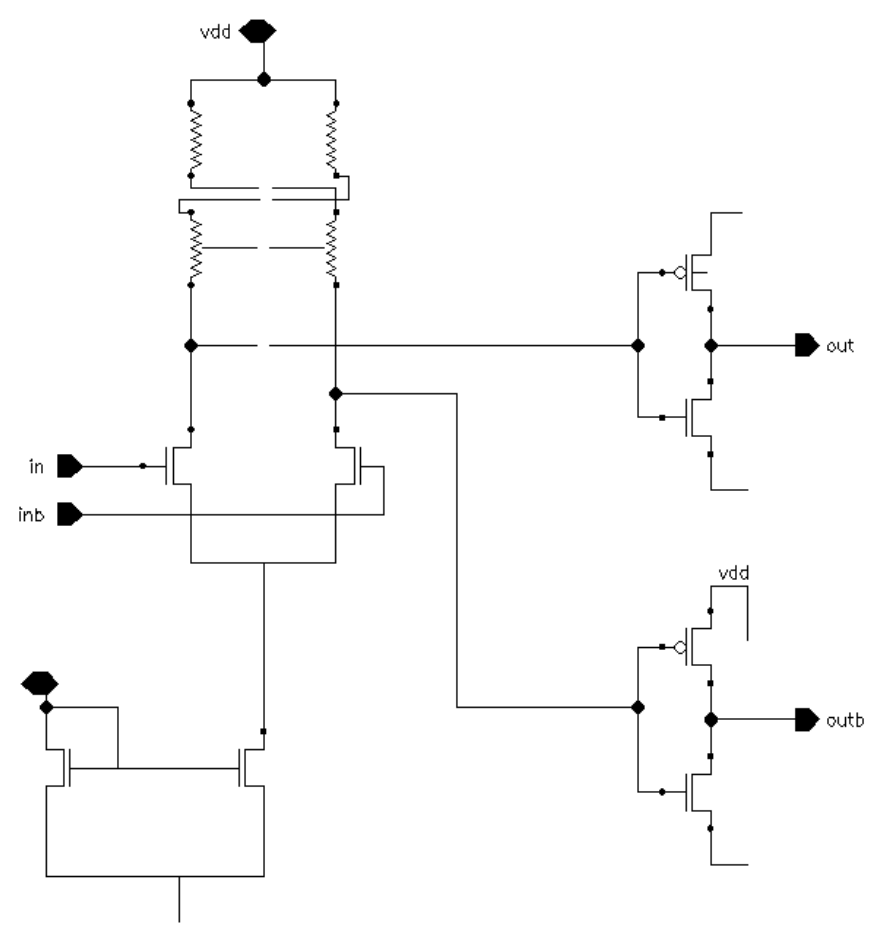

Figure 3.10: Schematic for the CML input buffer to drive the high-speed clock buffers. 


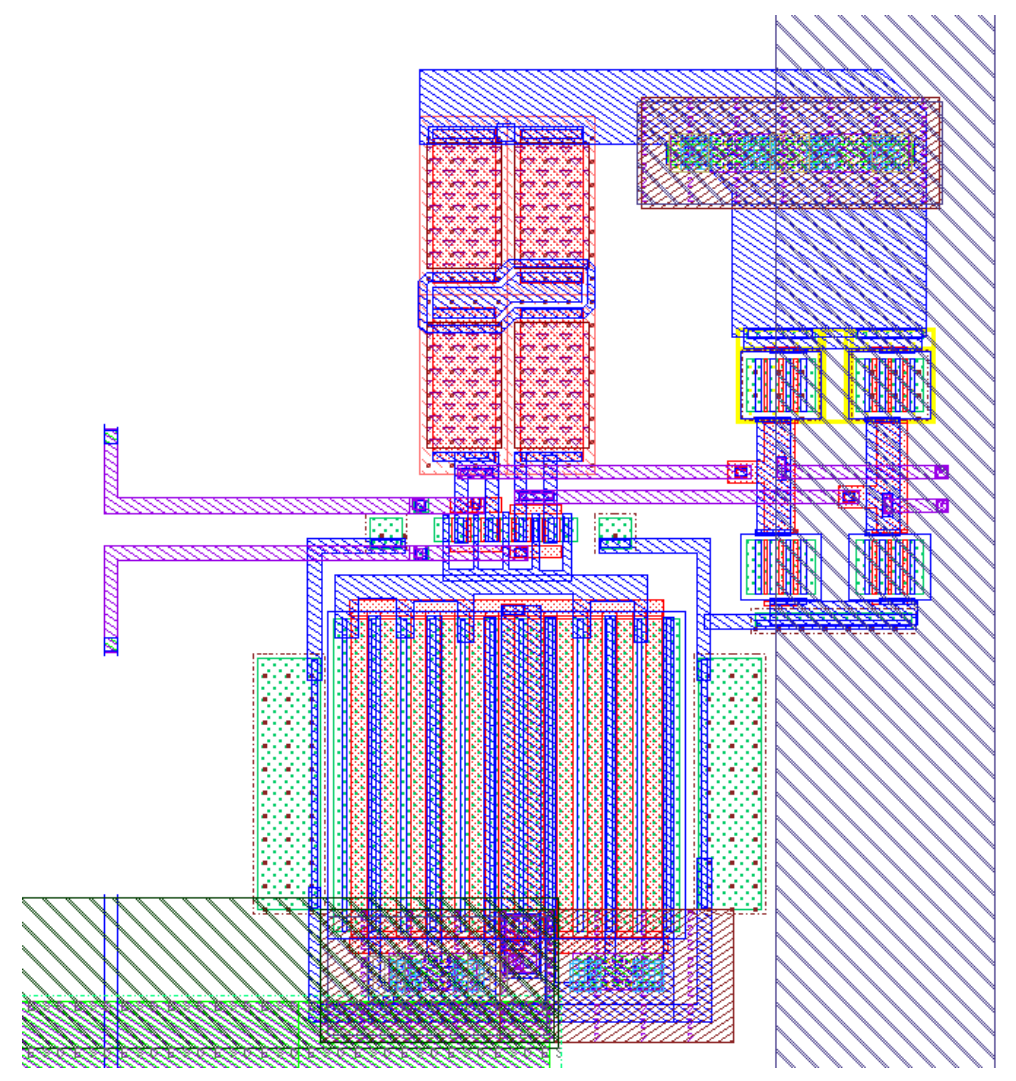

Figure 3.11: Corresponding layout for the CML input buffer. The CML load resistors are arranged in a common-centroid configuration. 
$\left(W_{P M O S}=3 \cdot W_{\min }\right)$. These values are then scaled by the above method for the case of the $\mathrm{C}^{2} \mathrm{MOS}$ cells. So, for example, the $\mathrm{C}^{2} \mathrm{MOS}$ NAND widths are scaled as follows:

- There are 3 series NMOS transistors; therefore the widths are set to $3 \cdot W_{\min }$.

- For the PMOS transistors, there are only two transistors in series, so the widths are twice the width of the single PMOS in an inverter, or six times the minimum transistor width in general, i.e. $2 \cdot W_{P M O S}=6 \cdot W_{\min }$.

The cells and their corresponding PMOS and NMOS widths (relative to minimum) are summarized in Table 3.2.

Table 3.2: Summary of the $\mathrm{C}^{2} \mathrm{MOS}$ standard cells and their transistor widths, relative to the minimum width.

\begin{tabular}{ccc}
\hline Cell & $W_{P M O S} / W_{\text {min }}$ & $W_{N M O S} / W_{\min }$ \\
\hline Inverter & 6 & 2 \\
Buffer & 3,6 & 1,2 \\
NAND2 & 6 & 3 \\
NOR2 & 9 & 2 \\
\hline
\end{tabular}

The layout of the standard cells used a track pitch of $12 \mu \mathrm{m}$. The schematics and corresponding layouts are shown in Figure 3.12 to Figure 3.15.

Two filler cells were also created so that the Encounter tool could fill the spaces between logic cells, and keep the $V_{D D}$ and $V_{S S}$ rails continuous. The larger of the two cells also included some dummy metal to help fix a problem with the copper dendrite DRC rule. Layouts for the filler cells are shown Figure 3.16 on page 50.

The cell layouts were then imported into Cadence Abstract Generator, to generate abstract views of the cells. The Abstract views are required for use with Cadence Encounter to perform placement of the logic cells and routing of the interconnect wiring between the cells.

\subsection{QAM Tx}

This section will detail the design of the QAM modulator, from the system level down to the register-transfer level of abstraction. 


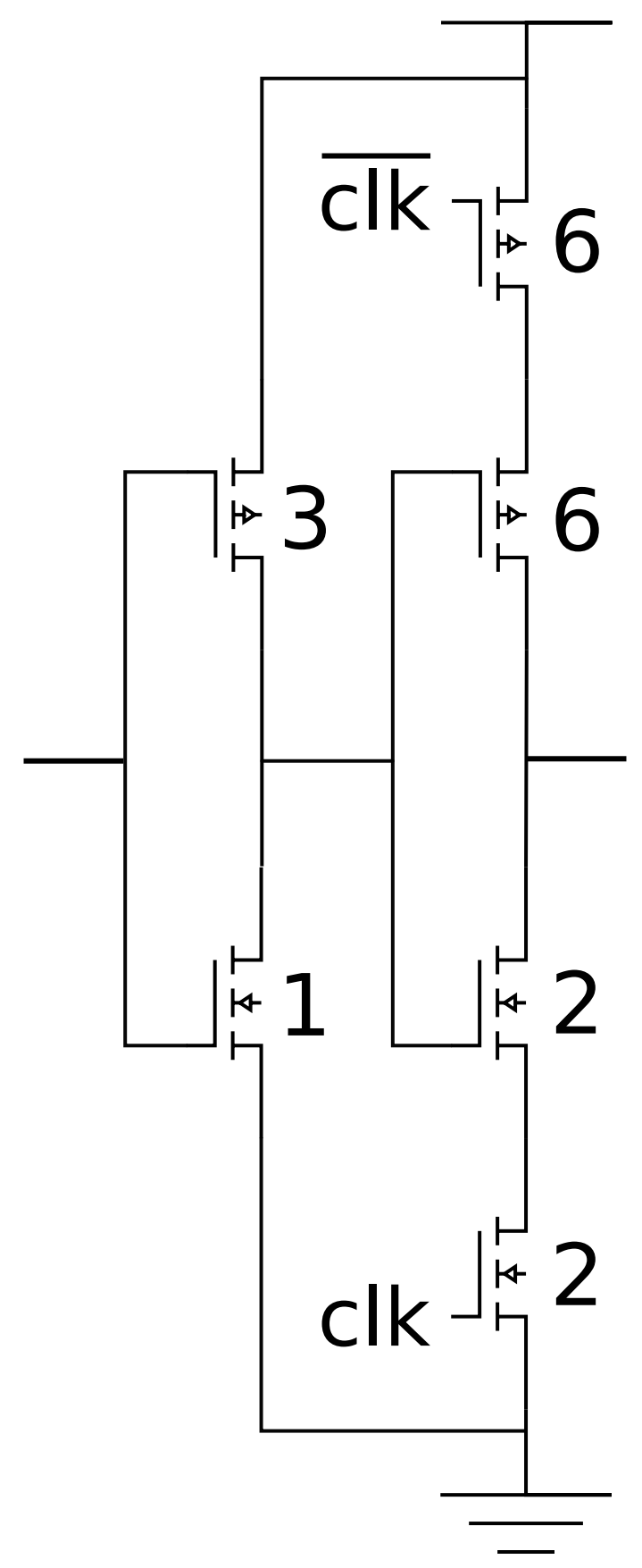

(a) Schematic. The relative transistor widths are annotated next to each transistor.

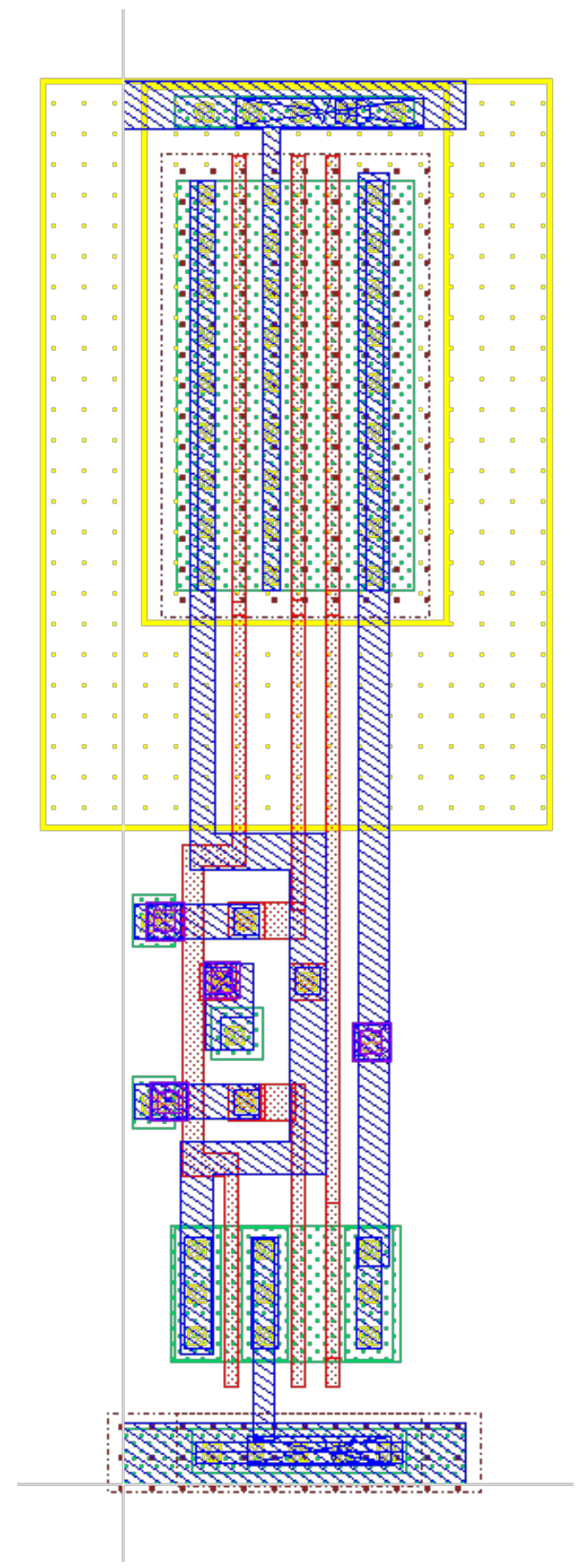

(b) Layout.

Figure 3.12: Schematic and layout for the $\mathrm{C}^{2} \mathrm{MOS}$ buffer. 


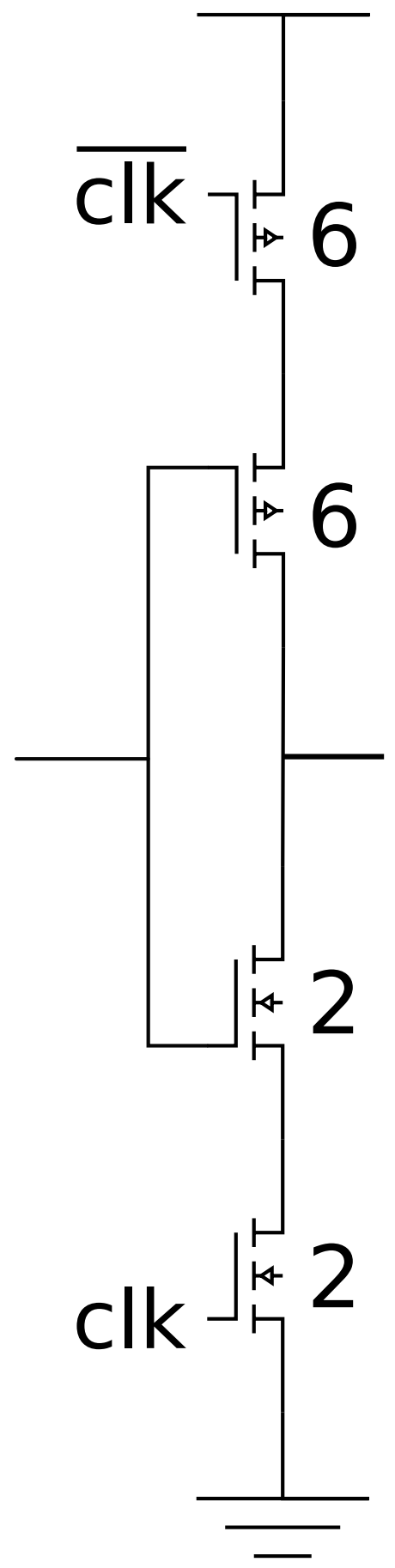

(a) Schematic. Also shown are the relative widths of each transistor.

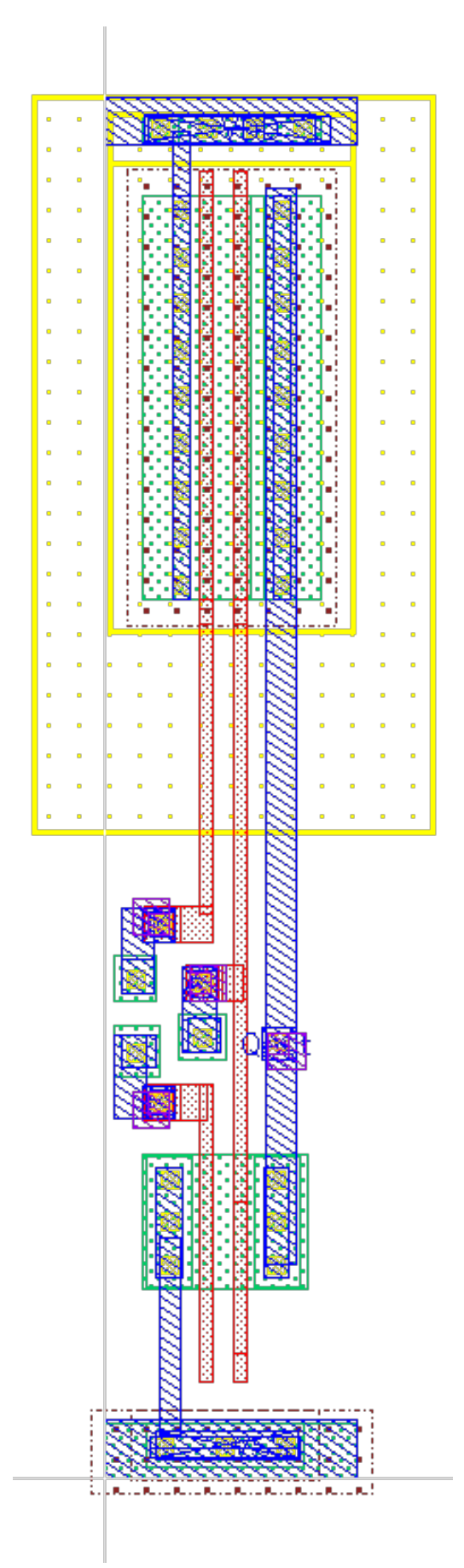

(b) Layout.

Figure 3.13: Schematic and layout of the $\mathrm{C}^{2} \mathrm{MOS}$ inverter. 


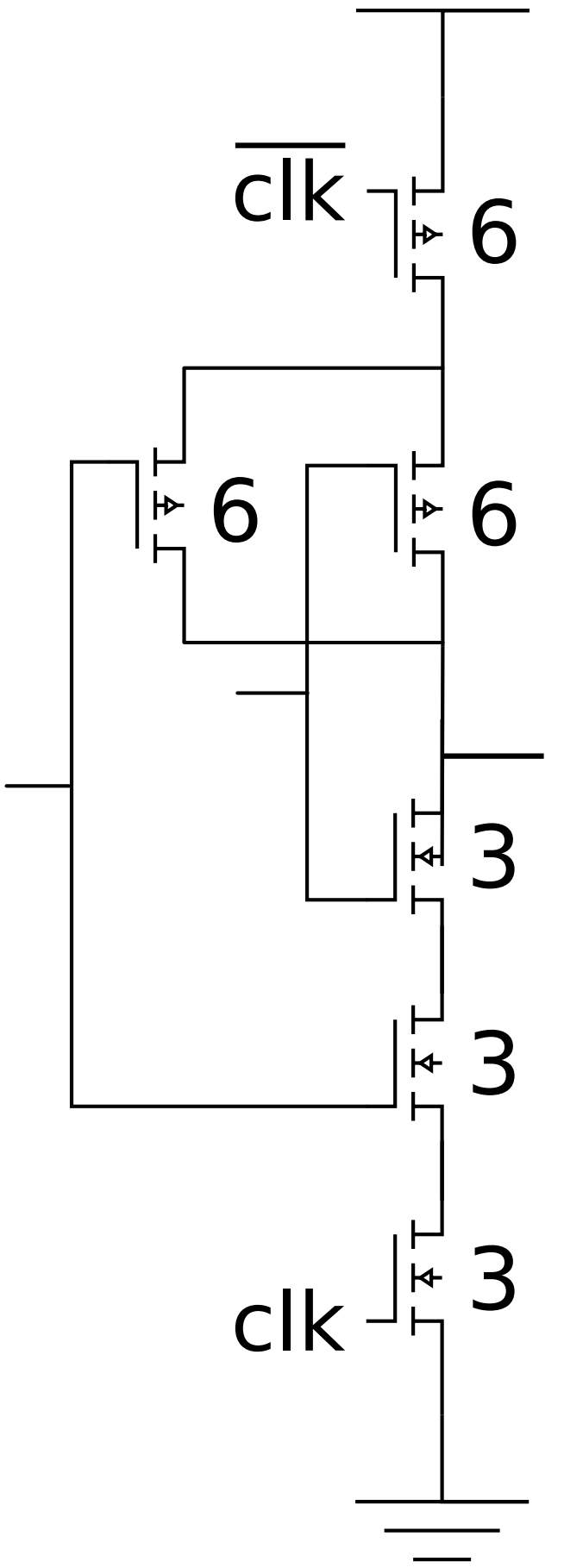

(a) Schematic. Relative transistor widths are also shown.

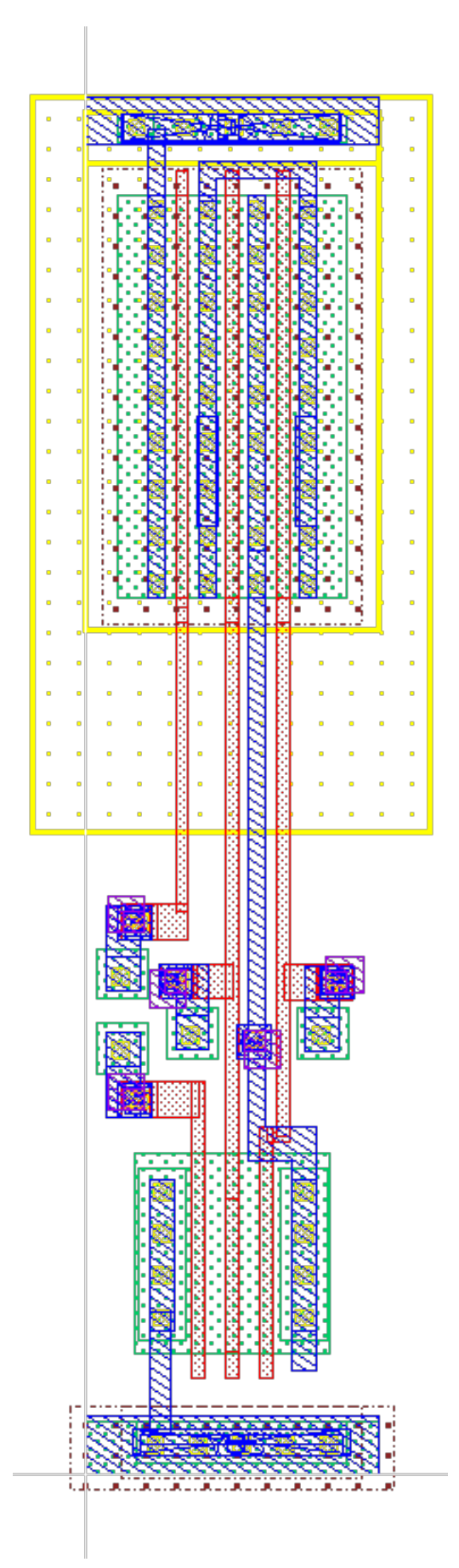

(b) Layout

Figure 3.14: Schematic and layout for the $\mathrm{C}^{2}$ MOS NAND2 cell. 


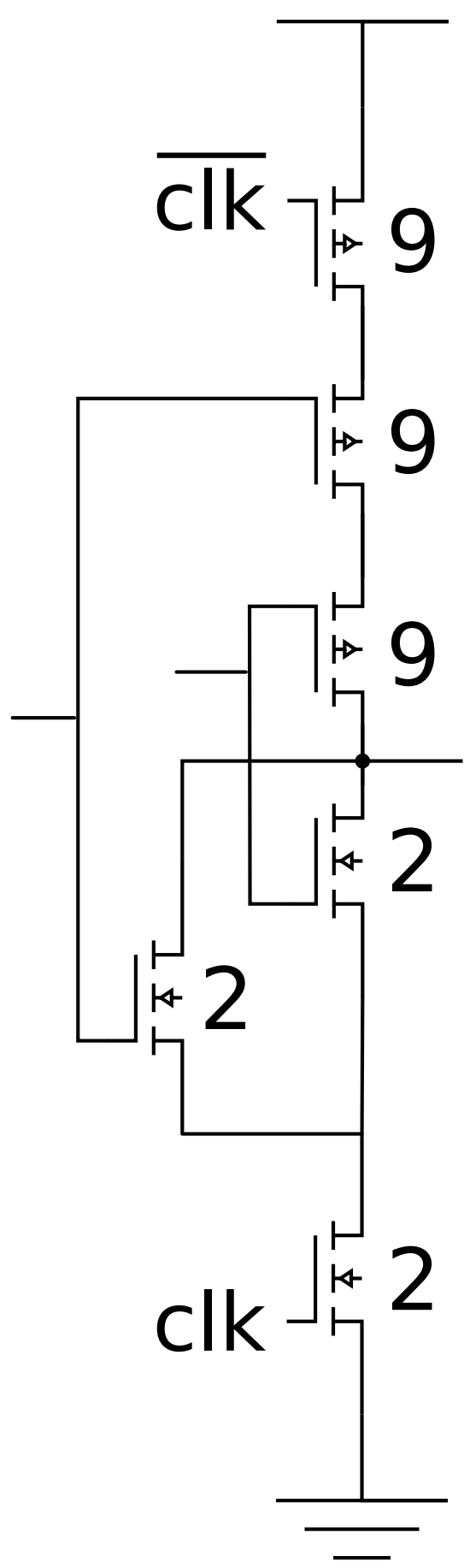

(a) Schematic, with relative transistor widths annotated.

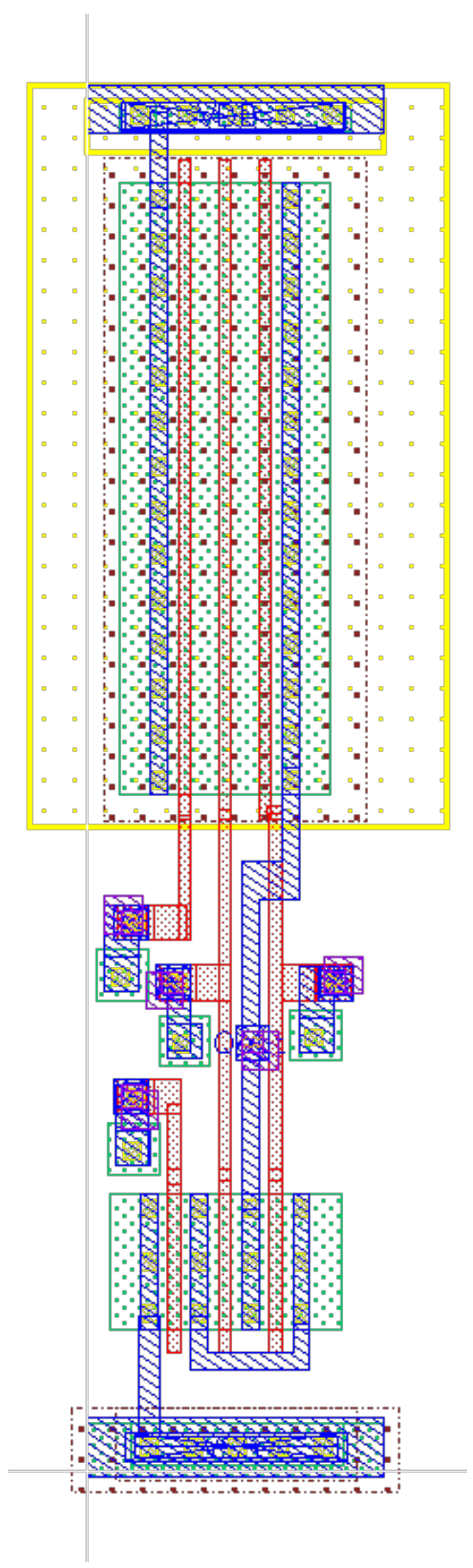

(b) Layout

Figure 3.15: Schematic and layout for the NOR2 $\mathrm{C}^{2} \mathrm{MOS}$ cell. 


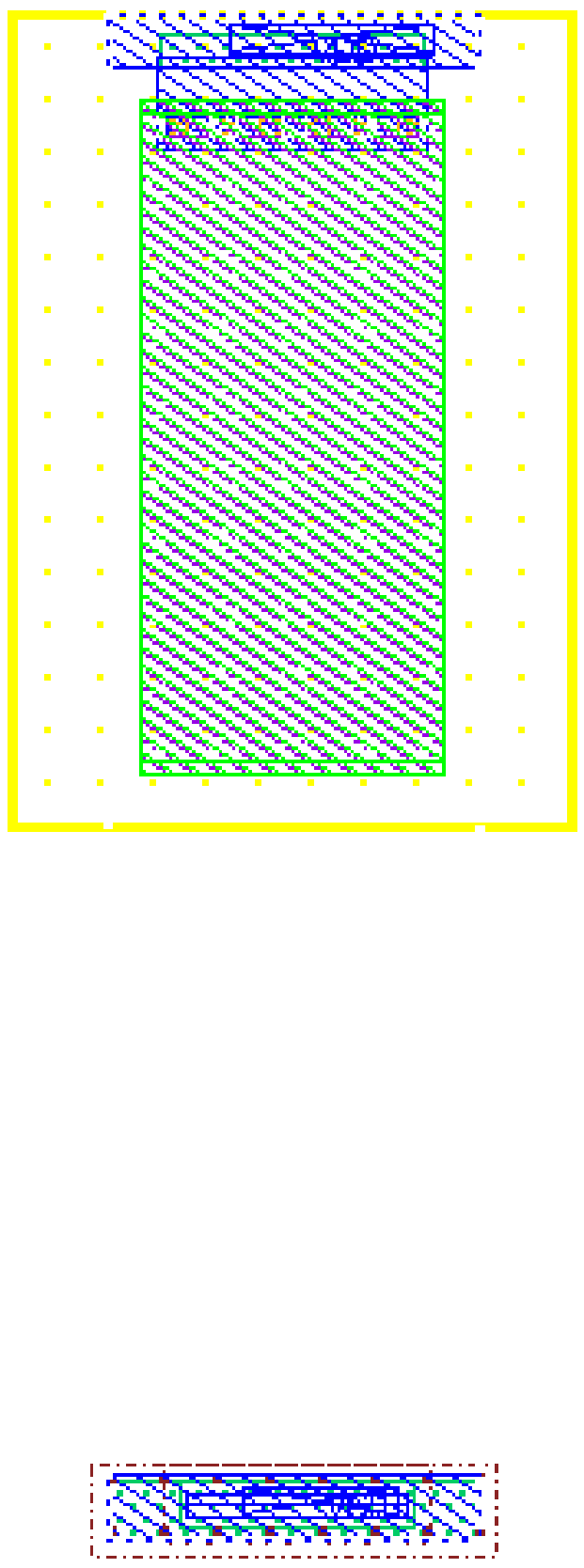

(a) Larger filler cell with extra dummy metal to mitigate copper dendrite formation.

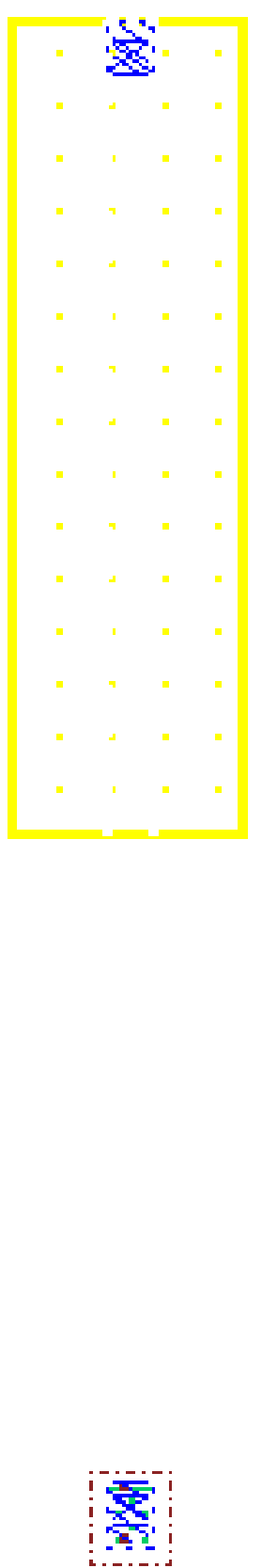

(b) Small filler cell for bridging gaps in the supply rails.

Figure 3.16: Layouts for the filler cells created. 


\subsubsection{System Level Design}

It was decided early on in the project to target the IEEE 802.11ad "WiGig" [31] standard; this was for a few reasons, namely:

- The standard includes a specification for a 16-QAM mode, which was considered to be complex enough to illustrate the usefulness of the proposed method, while being small enough to avoid an unreasonably large integrated circuit (IC) layout.

- The data rates are to be in the gigabit per second (Gbps) range, which would serve to illustrate the speed advantages of pipelining and $\mathrm{C}^{2} \mathrm{MOS}$. Specifically, for the single carrier 16-QAM mode being targeted, the data rate is specified to a maximum of 4.62 Gbps [31, Tab. 21-18].

- The standard is relatively new, which would make it relevant to the state of the art.

The block diagram for the baseband signal processing is shown in Figure 3.17.

Fixed point representation in Qn.m format, where:

$\mathrm{n}=$ number of integer bits

$\mathrm{m}=$ number of fraction bits

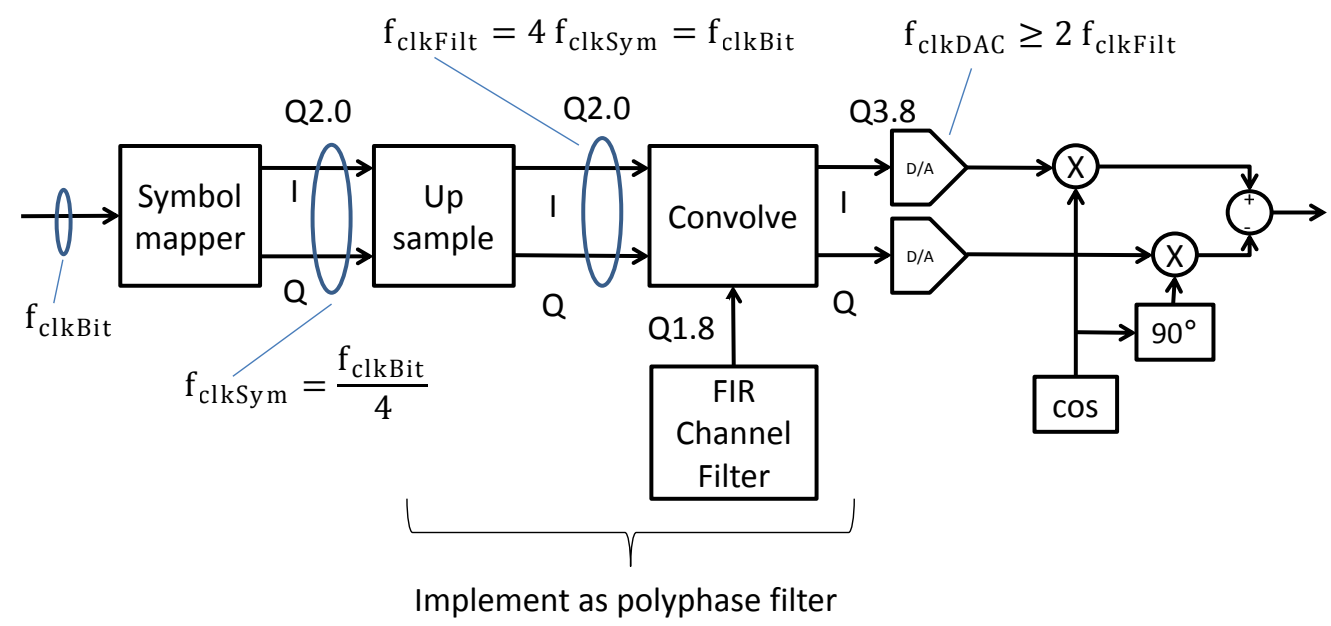

Figure 3.17: System block diagram for the QAM transmitter.

\section{Symbol mapping}

The 16-QAM symbol mapping was chosen to match that shown in the WiGig standard. The constellation showing the encoding of each 4-bit symbol to a constellation point is shown in Figure 3.18 on the next page. As can be seen, the first pair of bits 
are mapped to the $I$ level, which is defined to be one of $(-3,-1,+1$, or +3$)$; in a similar fashion the second pair of bits are mapped to $Q$.

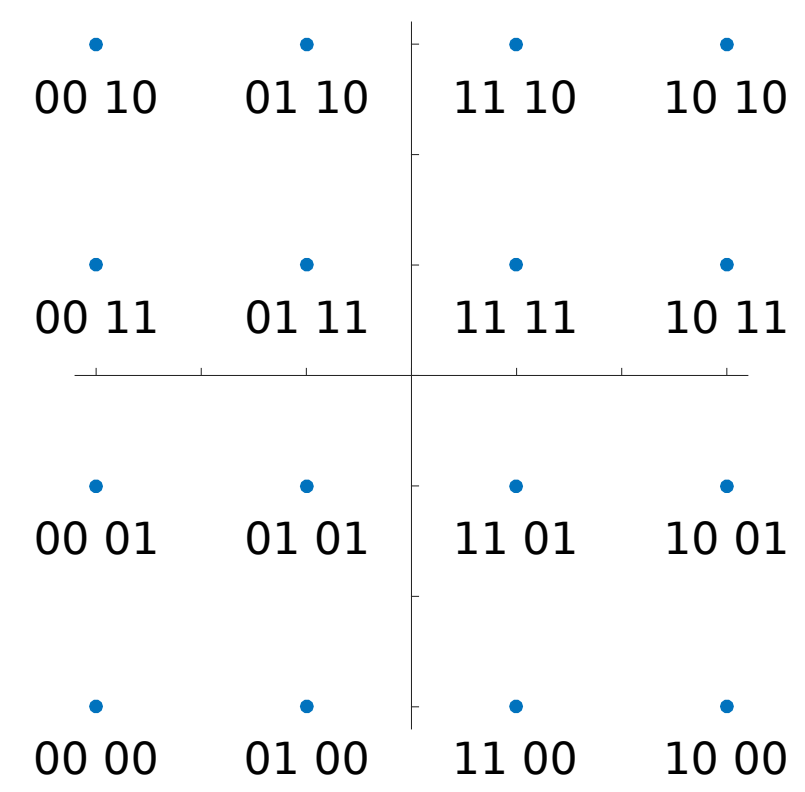

Figure 3.18: Symbol map for 16-QAM, adapted from the WiGig standard [31].

\section{$\pi / 2$ rotation}

The standard also requires the symbol mapping to undergo a $\pi / 2\left(90^{\circ}\right)$ rotation $[31$, Sec. 21.6.3.2.4.4]. Rotating the constellation periodically helps to prevent long durations of the same symbol being sent; this is primarily to reduce the peak-to-averagepower ratio (PAPR) [32]. Due to the non-linearities of some of the RF transmitter circuits, a signal with a high PAPR will experience more distortion.

\section{Pulse Shaping Filter}

Following the symbol mapping block is the pulse shaping circuit. Pulse shaping is used to reduce the bandwidth occupied by a digital baseband waveform, or as it is commonly thought of in the time-domain, to reduce the inter-symbol interference (ISI) [13, Sec. 3-6].

A frequently used filter that minimizes ISI is the raised cosine-rolloff filter (RCF). The name of the filter comes from the shape of its frequency response, which looks like a rectangular shape whose sides have been replaced by a segment of the cosine curve which creates a region called the transition band; for an example, see Figure 3.19 on the following page. The steepness of the transition band is determined by the rolloff factor $(r)$ of the filter, which is defined as the ratio of half the transition-band 
bandwidth $\left(f_{\Delta}\right)$ to the centre frequency of the transition band $\left(f_{0}\right)$ [13, Eq. 3-72]: $r=f_{\Delta} / f_{0}$.

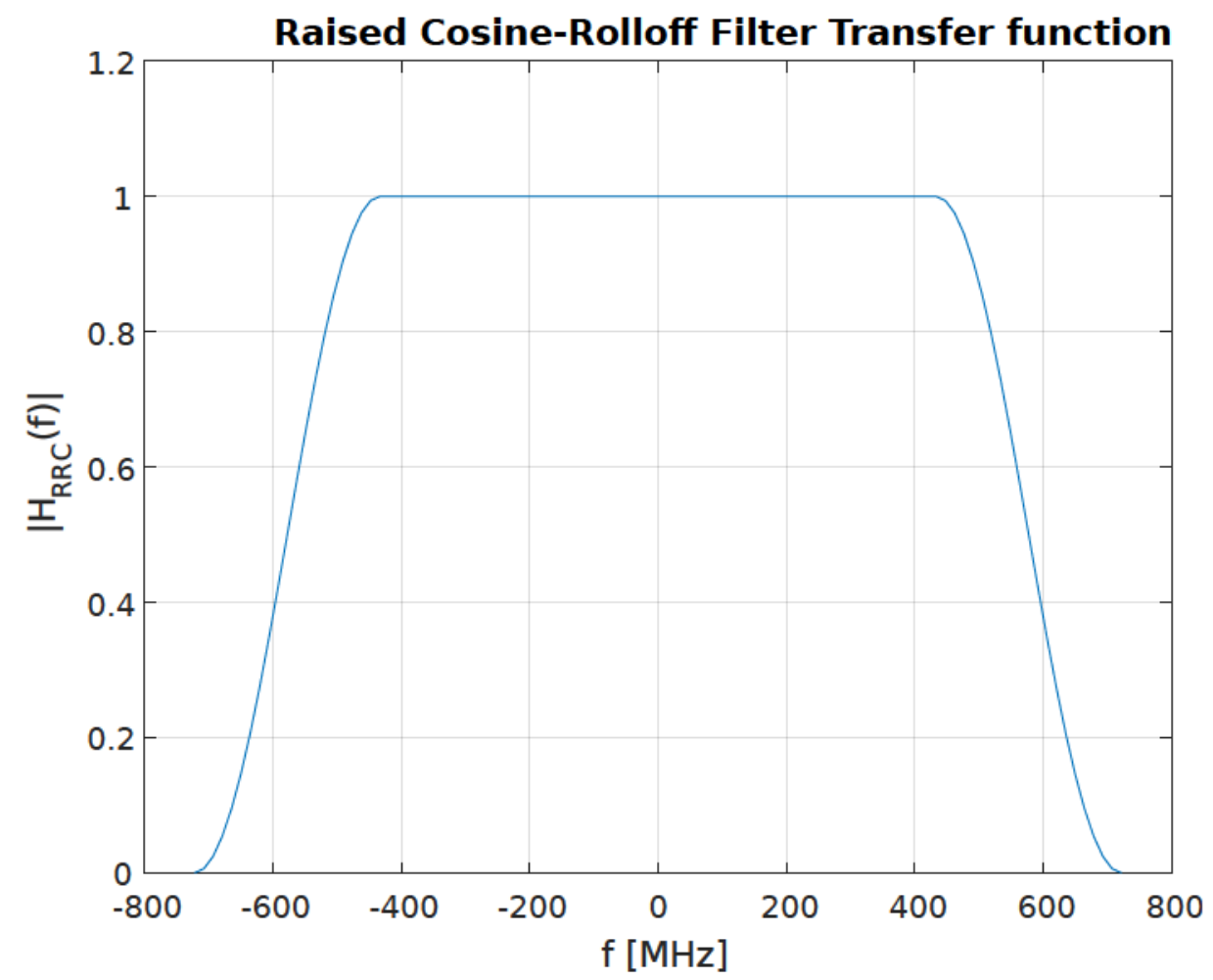

Figure 3.19: An example of a raised cosine rolloff filter's frequency response.

Additionally, reducing the impact of channel noise at the receiver requires a technique known as matched filtering [13, Ch. 6]. Stated simply, the matched filtering technique means to use the same filter at the transmitter and receiver. For the case of Gaussian noise, the RCF is easily converted to a matched filter by simply taking the square root of the RCF frequency response, thus forming a (square-)root raised cosine filter, RRCF, at the transmitter and receiver. Intuitively, it can be understood that this satisfies both the minimal ISI and matched filter requirements because:

- The signal from transmitter BB to receiver BB is filtered by two RRCFs, which is equivalent to being filtered by the product of the two RRCFs, i.e. an RCF; $\sqrt{H_{R C F}(f)} \cdot \sqrt{H_{R C F}(f)}=H_{R C F}(f)$

- The transmit and receive filter are the same RRCF, which in the time domain means they have the same pulse shape, thus the receive filter acts like a correlator to the transmit pulse.

The WiGig standard specifies an RRCF with a rolloff factor of $r=0.25$ for 
measuring the EVM. To simplify comparison to the standard, the design was chosen to be the same.

It was decided to implement the filter as a FIR type, since it has no feedback and therefore is more easily pipelined. As discussed in Section 2.2.1, a FIR filter is a DSP filter that implements the convolution of the input signal with a given impulse response's coefficients. First, the RRCF coefficients were calculated from the following equation [33, Sec. 6.8.1]:

$$
h_{R R C F}(t)=\frac{\sin \left(\pi \frac{t}{T_{S}}(1-r)\right)+4 r \frac{t}{T_{S}} \cos \left(\pi \frac{t}{T_{S}}(1+r)\right)}{\pi \frac{t}{T_{S}}\left(1-\left(4 r \frac{t}{T_{S}}\right)^{2}\right)}
$$

Where $t$ is the time, $T_{S}$ is the symbol period, and $r$ is the rolloff factor.

Since the target data rate is 4.62 Gbps for 16-QAM modulation, in which there are 4 bits per symbol, then the symbol rate would be 1.155 [Gsymbol/s], or 1.155 [Gbaud], or equivalently, the symbol period should be $T_{S} \approx 866 \mathrm{ps}$.

It was decided to use an up-sample (i.e. interpolate) by four on the I and Q stream before the signal passed to the pulse filter. An up-sample by four meant the FIR filter could be rearranged into a polyphase structure with four banks.

A model of the modulator was created in MATLAB code to aid in the design of the pulse shaping FIR filter.

The number of taps was increased until it was found that 39 coefficients gave a spectral response that fit within the spectral mask for the WiGig standard [31, Sec. 21.3.2], as well as exceeding the EVM requirement specified [31, Tab. 21-21], thus leaving some margin for the EVM requirements of the RF blocks.

Then, with the number of taps chosen, the fixed point format needed to be settled upon. The integer portion was decided to be 3 bits to allow for some overflow during the FIR calculations. And similar to the number of taps, the number of fractional bits was increased until the output spectrum still fit within the spectral mask and the EVM still met the specification with some margin left over for the RF blocks. As an example, the spectrum for 1200 symbols of random combinations of bits is shown in Figure 3.20 on the next page.

All of the design parameters of the QAM symbol mapping, rotation, and impulse shaping filter are summarized in the following table.

As introduced in Section 2.2.3, the structure of the filter can be compacted by taking advantage of the symmetry of a linear phase FIR filter, which applies for the case of a RRCF. Unfortunately, converting to a polyphase generally obscures the coefficient symmetry, but as revealed in [9] polyphase simply changes the left-right 


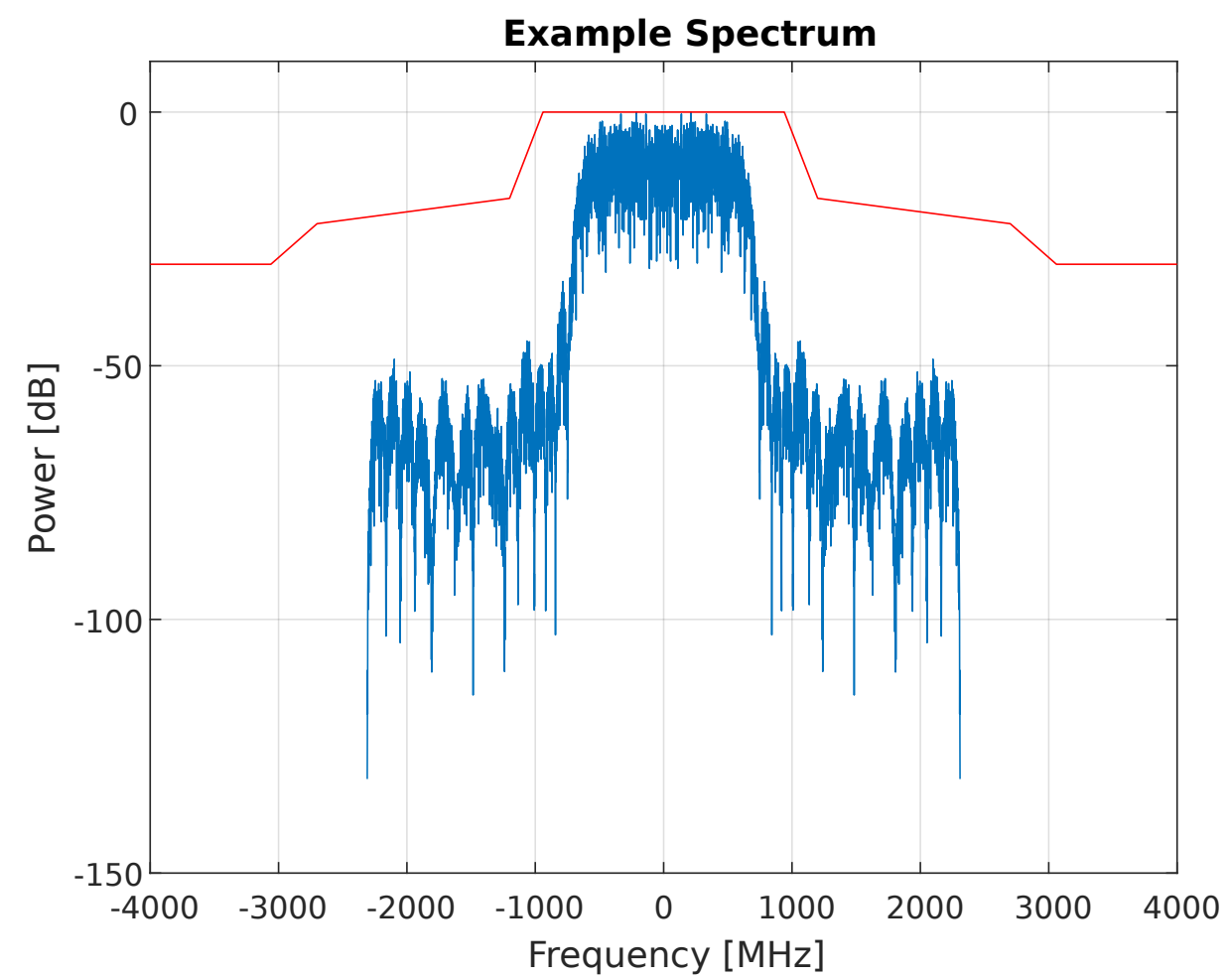

Figure 3.20: An example of the spectrum obtained for 1200 symbols worth of random bits, using the RRCF pulse shaping filter developed. Also shown is the transmit mask for WiGig [31, Fig. 21-1].

Table 3.3: Summary of design parameters for QAM transmitter.

\begin{tabular}{ll}
\hline \multicolumn{1}{c}{ Parameter } & \multicolumn{1}{c}{ Value } \\
\hline Modulation Scheme & $\pi / 2$-16-QAM \\
Pulse-shaping Filter & Root-raised cosine \\
Rolloff Factor $(r)$ & 0.25 \\
Data Rate & $4.62[\mathrm{Gbps}]$ \\
Symbol Rate $\left(T_{S}\right)$ & $1.155[\mathrm{Gbaud}]$ \\
Filter DSP Implementation & Polyphase FIR with restored co- \\
& efficient symmetry \\
Number of taps & 39 \\
Numerical format & Fixed \\
Fixed point representation & 3 integer, 8 fractional bits (Q3.8) \\
EVM & -24 dB \\
\hline
\end{tabular}


symmetry of the basic FIR filter into even-odd mirror pairs amongst the polyphase banks. New symmetric coefficients were synthesized from the polyphase coefficients as follows.

Additionally, as part of the procedure, the polyphase structure had to be modified to "undo" the symmetric coefficient transformation. This new polyphase, symmetric coefficient structure, is shown in Figure 3.21 on the following page.

\subsubsection{Behavioural Level Design}

Having finalized the system-level design, Verilog hardware description language (HDL) code was written to implement the behaviour of the modulator. Once complete, the design was simulated with Mentor Graphics' ModelSim simulator to verify the functionality of the circuit. A testbench was created to apply a stimulus of data bits from a file that was generated with MATLAB. The testbench then saved the output of the modulator to a new file. This new file was then loaded into MATLAB and the results from the ModelSim simulation were confirmed to be the same as the MATLAB simulation.

\subsubsection{Synthesis and FPGA Implementation}

The behavioural Verlilog RTL was then synthesized using Synopsys' Design Compiler tool, using a small library of cells (namely, a two-input NAND, two-input NOR, an inverter, and a buffer). These cells were intended for use with the $\mathrm{C}^{2} \mathrm{MOS}$ cells developed. Unfortunately, after a preliminary place and route of the design using Cadence's Encounter tool, it was discovered that the transmitter would not fit within the integrated circuit size awarded by the Canadian Microelectronics Corporation (CMC) (1 by $1.5 \mathrm{~mm}$ ). So the design was retargeted to a Xilinx Virtex 5 FPGA, model XC5VFX200T, available on the ML-525 evaluation board. The new design flow for the FPGA still used the Design Compiler with the small library of cells to avoid having to rewrite the Perl script to use the Xilinx Virtex 5 cells. The flow is summarized as follows:

1. Synthesize with Synopsys Design Compiler.

2. Process Verilog netlist with Perl.

3. Re-synthesize Perl processed netlist with Xilinx ISE. 


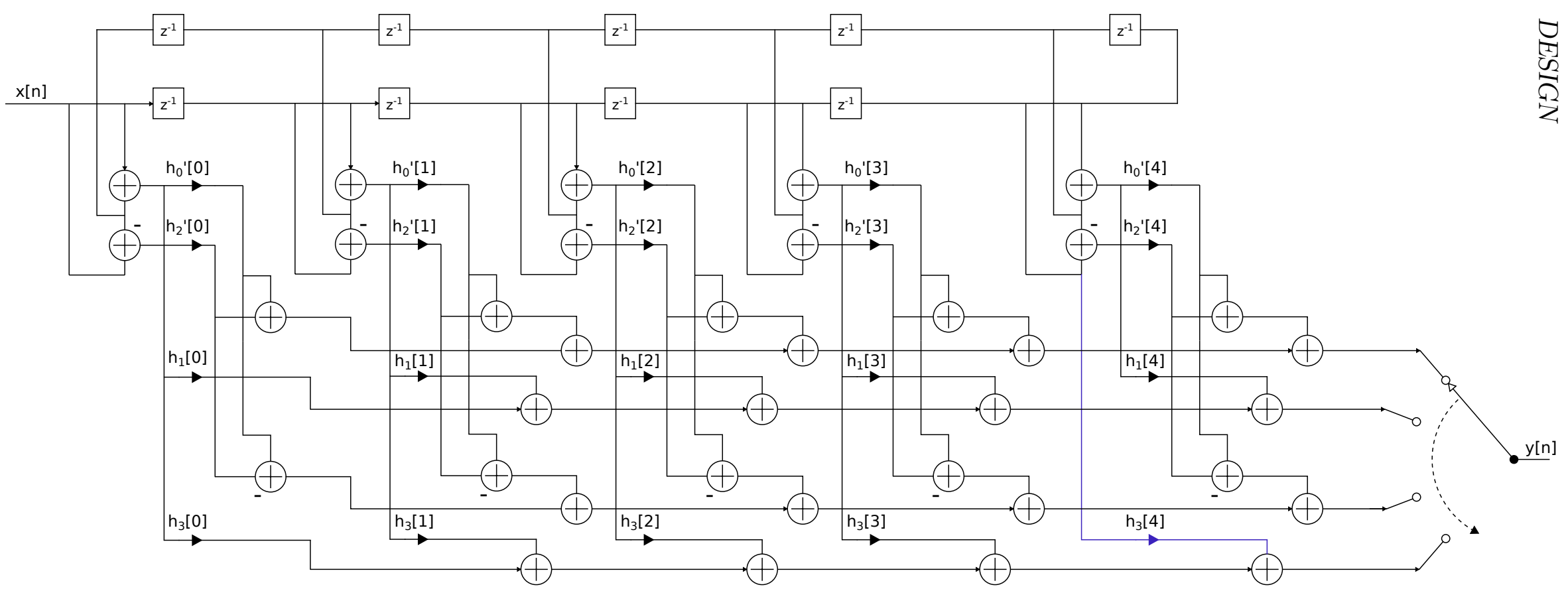

Figure 3.21: The polyphase FIR filter, modified for coefficient symmetry. 


\subsection{CIC Filter}

It was decided that since the QAM modulator would not fit in the $1.5 \mathrm{~mm}^{2} \mathrm{IC}$ die size awarded by CMC, a different DSP filter would be implemented. A CIC filter was implemented as it is relatively compact. This section describes the design of the CIC filter at the system down to the gate level.

\subsubsection{System Level Design}

CIC filters were introduced in Section 2.2.2. One primary application is in the antialiasing or anti-imaging filters required at an up-sample (interpolation) or downsample (decimation) point in a DSP chain. They are relatively compact filters that use few storage elements (i.e. FF registers) and only require adders and subtracters. The FFs are combined with adders and subtracters to form integrator and comb stages.

In designing the CIC filter, the number of integrator and comb stages had to be selected. And in a similar manner to the FIR filter, the word size for conversion to fixed point representation had to be chosen.

Based on the aforementioned IC size constraints, it was found that two comb and two integrator stages, all with a 10-bit word width, would fit well within the IC die size. The system block diagram of this CIC filter is shown in Figure 3.22.

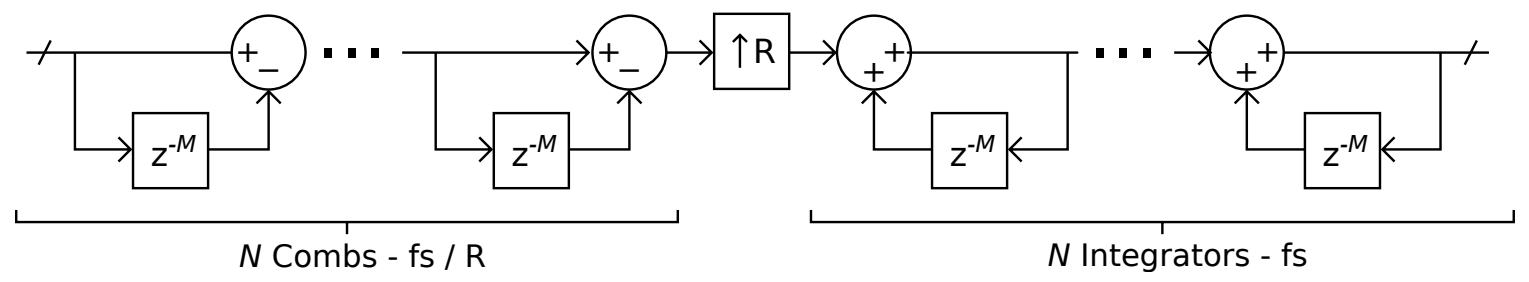

Figure 3.22: System block diagram of CIC filter.

The filter response for this implementation, with an up-sample by 10, is plotted in Figure 2.4 on page 14.

\subsubsection{Behavioural Design}

The process for the behavioural design of the CIC filter was similar to the QAM modulator. The procedure was as follows.

1. The design was written in Verilog, and its function was confirmed against the MATLAB simulation using ModelSim from Mentor Graphics. 


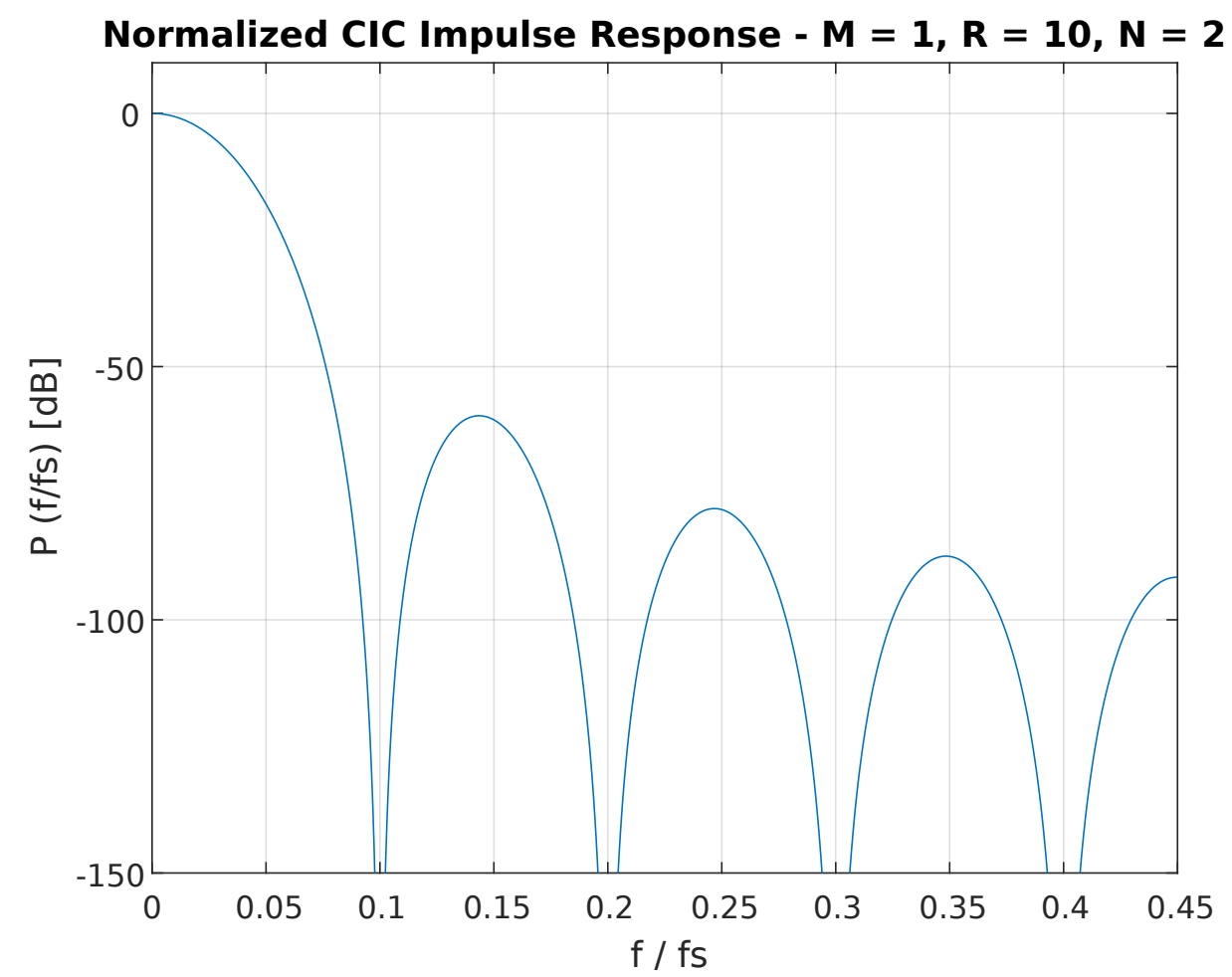

Figure 3.23: Frequency response of the CIC filter that was designed.

2. The design was then synthesized and mapped to the $\mathrm{C}^{2} \mathrm{MOS}$ cell library using Design Compiler, resulting in a Verilog netlist of only the $\mathrm{C}^{2} \mathrm{MOS}$ cells.

3. This netlist was then processed by the Perl script to create a new Verilog netlist with all path delays balanced.

\subsubsection{IC Design and Layout}

After completing the behavioural level of the design, the IC design and layout work was carried out.

\section{Floorplanning}

First, some basic floorplanning was done for the whole chip, and from that the pad frame was based. The digital core of the CIC filter would then fit in the centre of the pad frame. The remaining space between was left for wire routing going out to the pads and metal fill.

Due to the limited size of the chip die, the number of pads that could be used was limited. Therefore, it was decided to use a rudimentary boundary scan-like chain to write in the inputs and read out the outputs of the CIC filter. The chain was 
designed as a parallel loadable shift register. This required a pad for the shift register data input, another for the output, a clock, and a latch enable to activate the parallel load / read operation; 4 pads altogether. A differential clock signal was used to drive the high-speed logic; the pads for this were set up in a ground-signal-signal-ground (GSSG) configuration, requiring another 4 pads. The two lowest bits of the CIC filter output were also sent off chip, requiring a GSSG output each. Table 3.4 on page 62 summarizes all of the pads needed for the CIC chip. In all, it was found that 24 pads would be needed, which would just fit within the $1.5 \mathrm{~mm}^{2}$ die.

\section{Place and route}

Having generated a final Verilog netlist, the digital core of the design needed to be created. To create the layout for the core, the Encounter software from Cadence was used to place the $\mathrm{C}^{2} \mathrm{MOS}$ cell abstracts and route the connections for the cells. Unfortunately, due to scheduling, a proper clock distribution strategy was not developed, and an attempt to have Encounter place and route a clock buffer cell was unsuccessful. As an alternative, the Verilog netlist was manually edited to include a fanout-of-four (FO4) clock buffer cell, whose schematic is shown in Figure 3.24. The FO4 cell was added at each level in the abstraction hierarchy. The completed core layout is shown in Figure 3.25 on the next page.

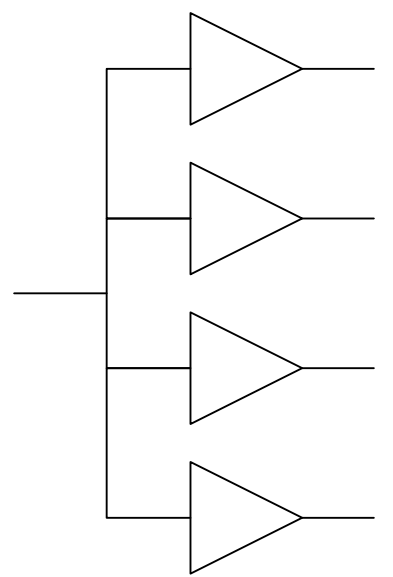

Figure 3.24: Schematic for the fanout-of-4 clock buffer cell.

Once the place and route $(\mathrm{PnR})$ step was completed, the core was exported to the industry standard LEF / DEF file formats. These files were then imported into Cadence's Virtuoso suite. With the core now in Virtuoso, it could be placed inside the pad frame that was created. From that point, the final connections to the I/O pad buffers were made. Then, the design rule check (DRC) software, with the predefined 


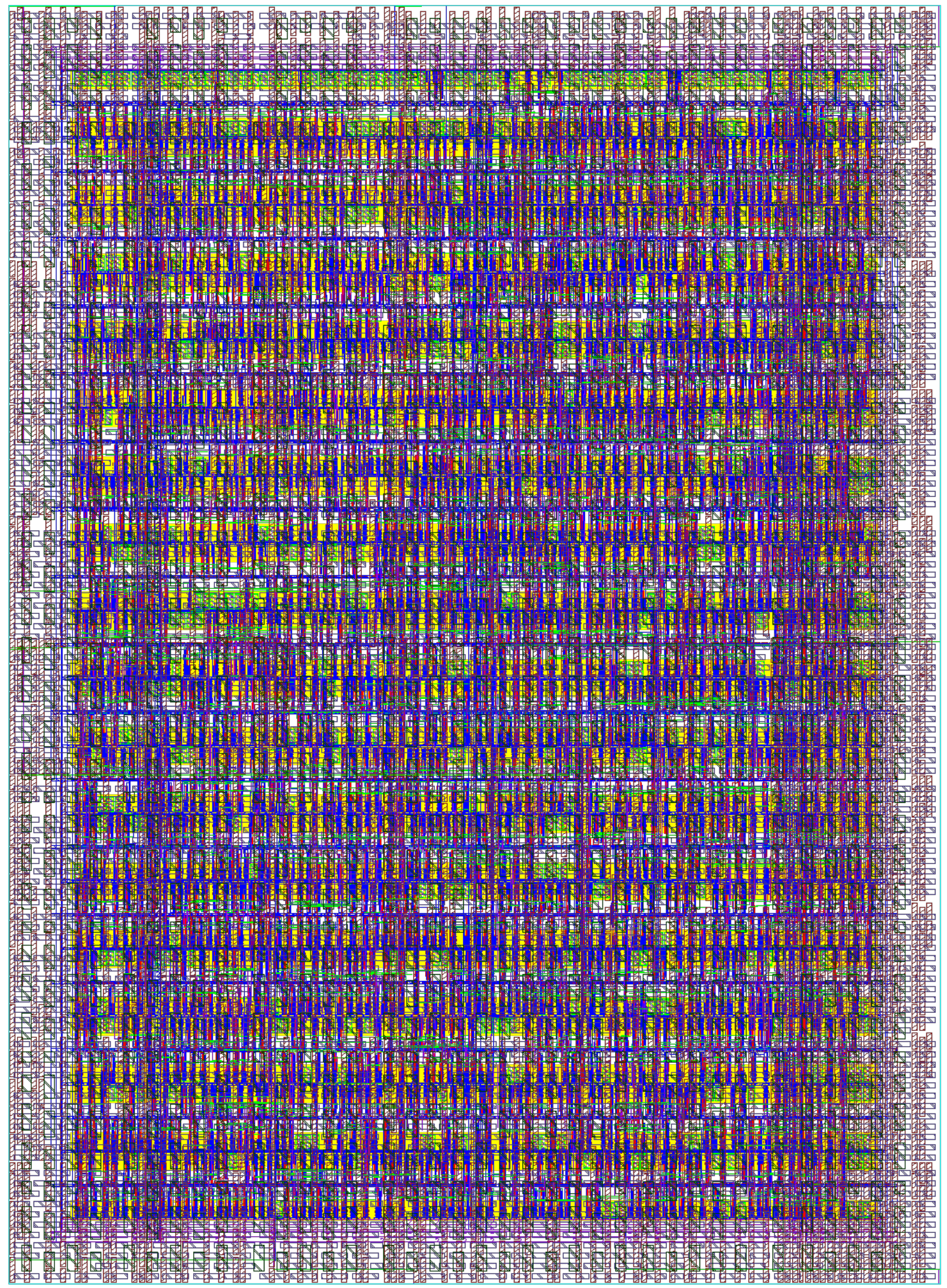

Figure 3.25: Layout of the digital core of the CIC filter. 
Table 3.4: A list of the pads used for the CIC chip.

\begin{tabular}{lclc}
\hline \multicolumn{1}{c}{ Inputs } & \multicolumn{2}{c}{ Outputs \& Power } \\
\multicolumn{1}{c}{ Pin Function } & \# Pads & Pin Function & \# Pads \\
\hline High-speed clock & 4 & output bit 0 & 4 \\
Comb clock & 1 & output bit 1 & 4 \\
Chain clock & 1 & & 1 \\
Chain data in & 1 & Chain data out & 3 \\
Latch enable & 1 & & 2 \\
Integrator zero & 1 & $V_{D D}$ & 14 \\
Current reference & 1 & $V_{S S}$ & \\
\hline Total & 10 & & \\
Absolute Total & 24 & & \\
\hline
\end{tabular}

IBM $130 \mathrm{~nm}$ rule decks, was run to confirm the layout rules for fabrication were met. The Verilog netlist had also been imported into Virtuoso to allow a layout versus schematic (LVS) check to be performed.

Having passed the LVS check, the design was exported to a GDSII stream file, which was then uploaded to the CMC servers to be sent for fabrication. The final layout is shown in Figure 3.26 on the following page.

\subsection{Summary}

This chapter presented the design process taken in developing the pipelining algorithm, and applying it to the design of a QAM transmitter on an FPGA and a CIC filter integrated on a $130 \mathrm{~nm}$ CMOS process. The next chapter will provide the results of testing both circuits in the lab. 


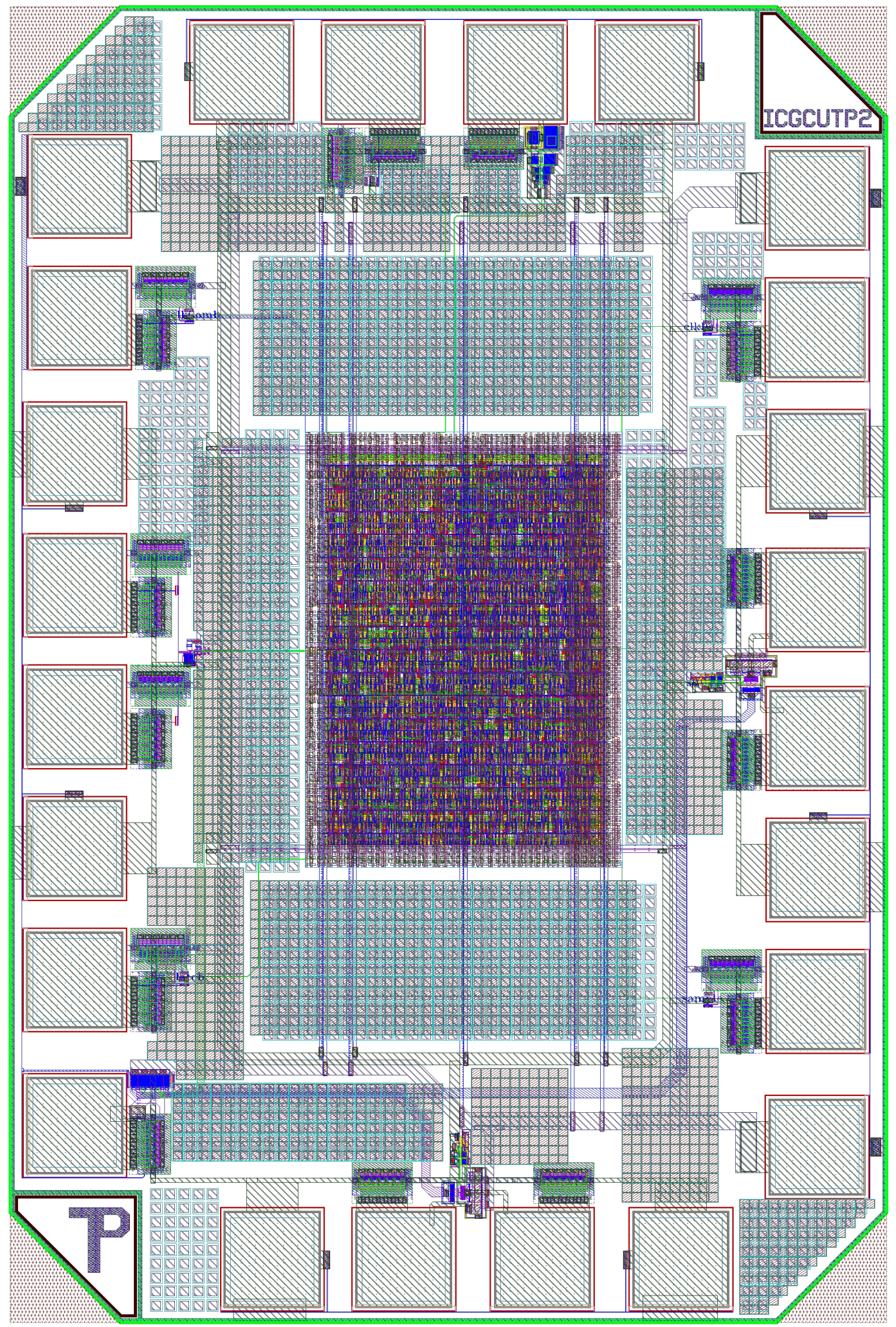

Figure 3.26: Layout of the entire CIC filter IC. 


\section{Chapter 4}

\section{Testing}

This chapter presents the results from testing the original output buffers, the CIC filter design, and the QAM transmitter.

\subsection{Buffers}

The test chip for the first buffer design contained various I/O blocks and was used to become familiar with the design, layout and testing process. As well, these were necessary blocks for the final circuit and successful testing of these provided some confidence in the whole process. The test chip, whose photomicrograph is shown in Figure 4.1 on the next page, was sent out for fabrication in January 2013. The completed chips were received, and testing started, in July 2013. Testing confirmed functionality with operation up to about $2 \mathrm{GHz}$, which showed that the buffers did operate, but not at the target rate of $5 \mathrm{GHz}$. Based on this result, the high-speed output buffers were redesigned using a different technique. Further information can be found in Section 3.2.1. However, these output buffers were re-used for the lower speed outputs used in the CIC test chip.

\subsection{CIC Filter}

The second test chip, sent out for fabrication in April 2014, made use of high-speed digital logic using Clocked CMOS ( $\left.\mathrm{C}^{2} \mathrm{MOS}\right)$ pipelining. As explained in earlier chapters, this technique has the potential to allow multi-Gbps rates in older (and hence cheaper) process nodes, in this case, $130 \mathrm{~nm}$ CMOS. The original target as an example application was for 16-QAM for WiGig (802.11ad). However, due to the relatively 


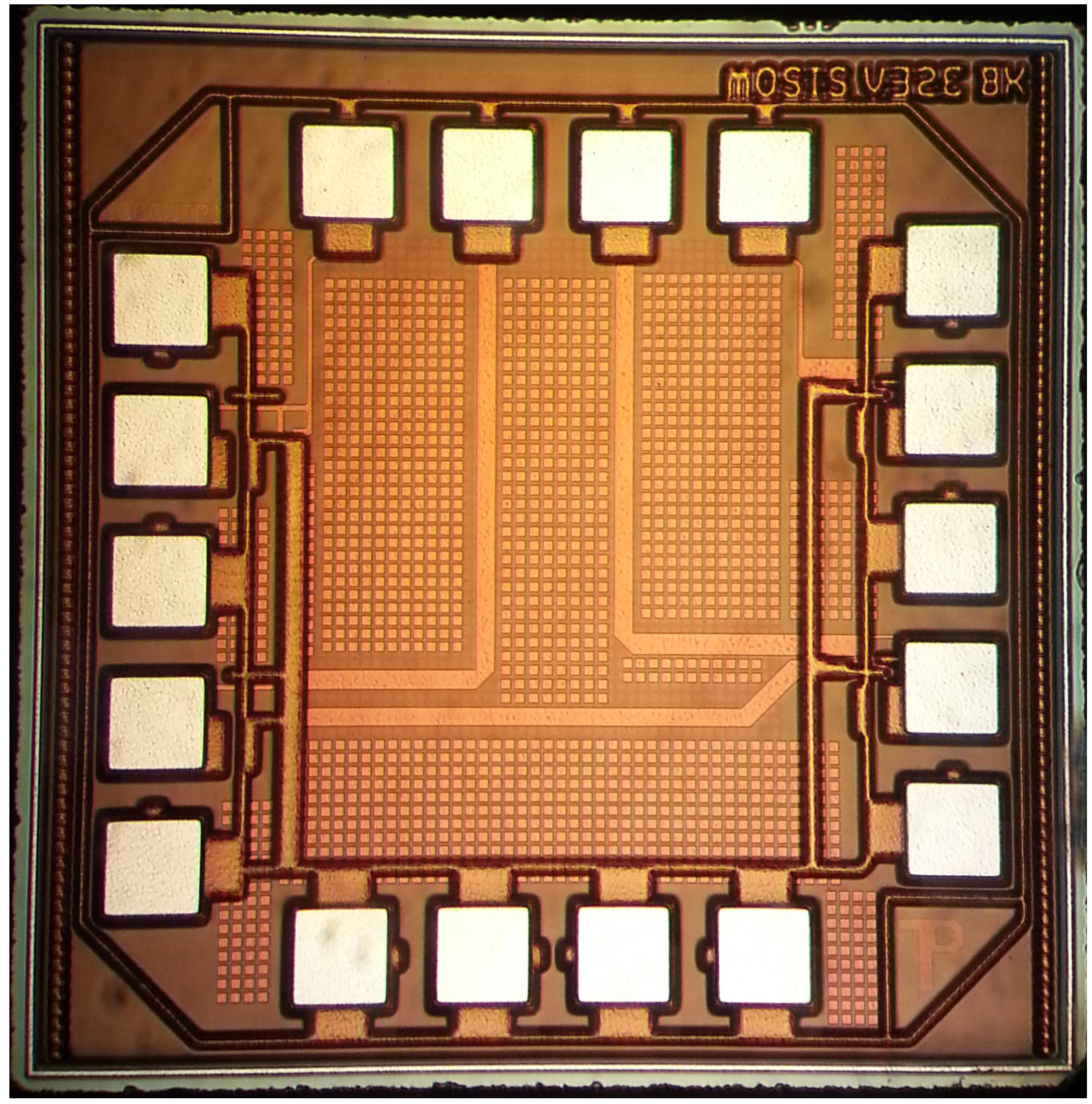

Figure 4.1: Photomicrograph of the buffers chip. The size of the die is 1 by $1 \mathrm{~mm}$. 
small chip size granted ( 1 x $1.5 \mathrm{~mm}$ ), it was decided to implement the Cascaded Integrator Comb (CIC) interpolation filter, but it would still be designed to operate at WiGig rates. The photomicrograph of the CIC test chip can be seen in Figure 4.2 on the following page.

\subsubsection{Test Setup}

The chip was wire bonded into a CFP24 package by CMC, and the CFP24 test jig available from CMC was used to connect signals to the chip. The test jig provides convenient connection to the signal lines through SMA connectors. The package mounted into the test jig is shown in Figure 4.3 on page 68.

To aid in testing the chip, a Verilog module which applied stimulus inputs and read back results from the device under test (DUT) was created. A Virtex-5 ML-525 FPGA evaluation board was used to implement the stimulus and control function. The ML-525 evaluation board was chosen due to its available SMA connectors which made interfacing to the CFP24 test jig simple. A set of SMA cables was used to connect the high-speed outputs to an Agilent $16 \mathrm{GHz}$ Digital Signal Analyzer (DSA), model DSA-X 91604A, which was borrowed from CMC through their equipment pool loan service. The FPGA clock was sourced from an on-board synthesizer, whose reference was from a Rigol DG1002A function generator.

A picture of the test setup can be seen in Figure 4.4 on page 69, and a schematic representing the test setup is given in Figure 4.5 on page 70 .

When the chip was attempted to be tested, it was noticed that the scan chain was not functioning correctly. For a stream of bits input, the output was toggling only occasionally. Due to scheduling constraints during the design, simulation of the scan chain had been overlooked. However, upon simulating the scan chain, the same output behaviour was observed in simulation as in the lab. Further simulation probing into the internal nets revealed the source of the problem. The clock signal has very slow rising and falling edges as seen in Figure 4.6 on page 71 and as a result both the clock $(c l k)$ and its complement $c l k_{b}$ are active at the same time, resulting in the signal racing through several FFs on each rising edge. This can be seen in Figure 4.7 on page 71 where on each edge, the changing data value passes along through several series FFs in the chain.

In an attempt to save power and layout area, it was decided not to buffer the clock net for the flip-flops in the scan chain. This is the reason for the slow edges on the clock signal. The problem could easily have been prevented by including clock buffers, 


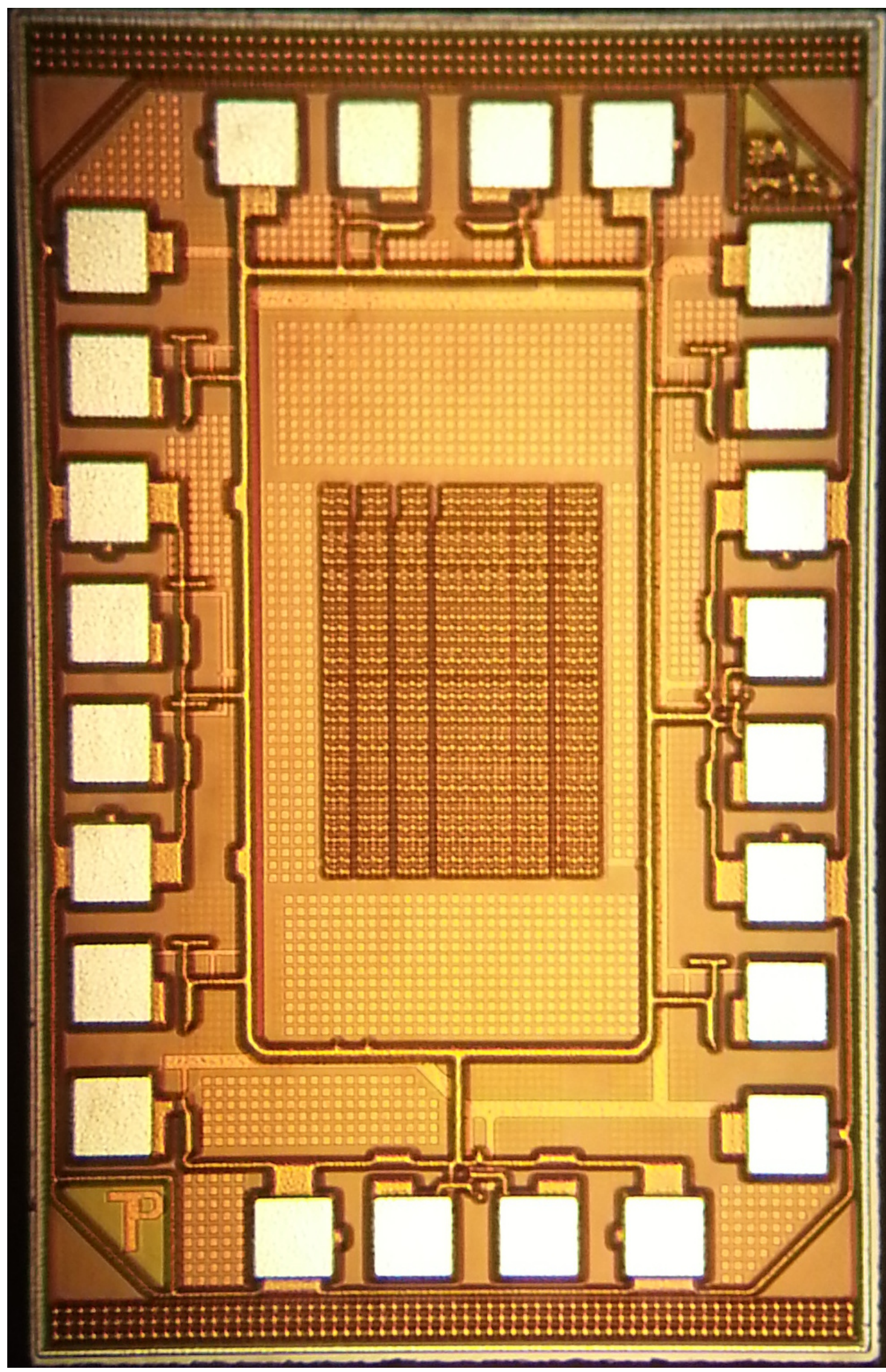

Figure 4.2: Photomicrograph of the CIC chip. The die size is 1 by $1.5 \mathrm{~mm}$. 


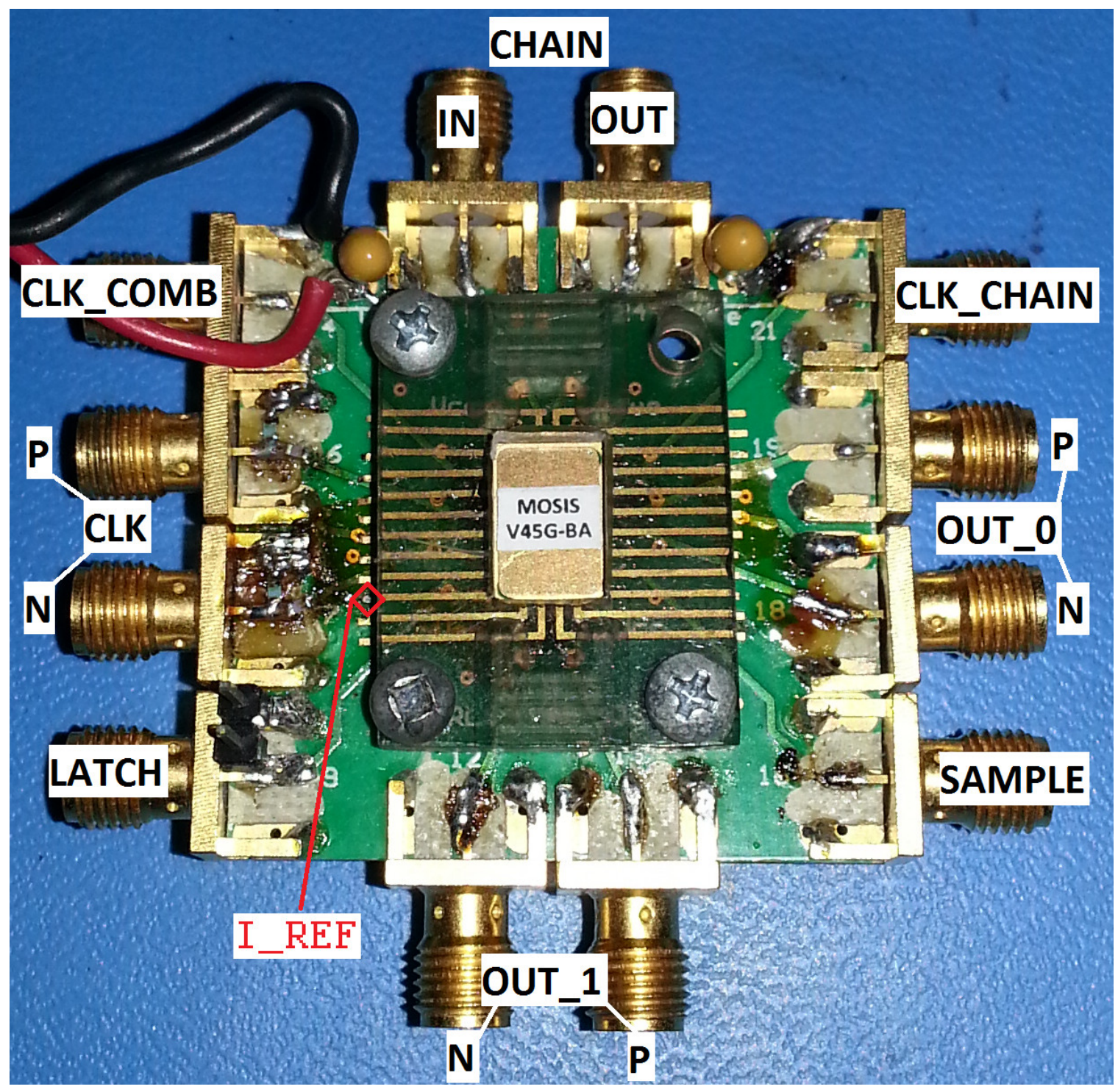

Figure 4.3: The CFP24 test jig PCB with the packaged CIC chip inserted. The relevant signals are labelled on their corresponding SMAs. 


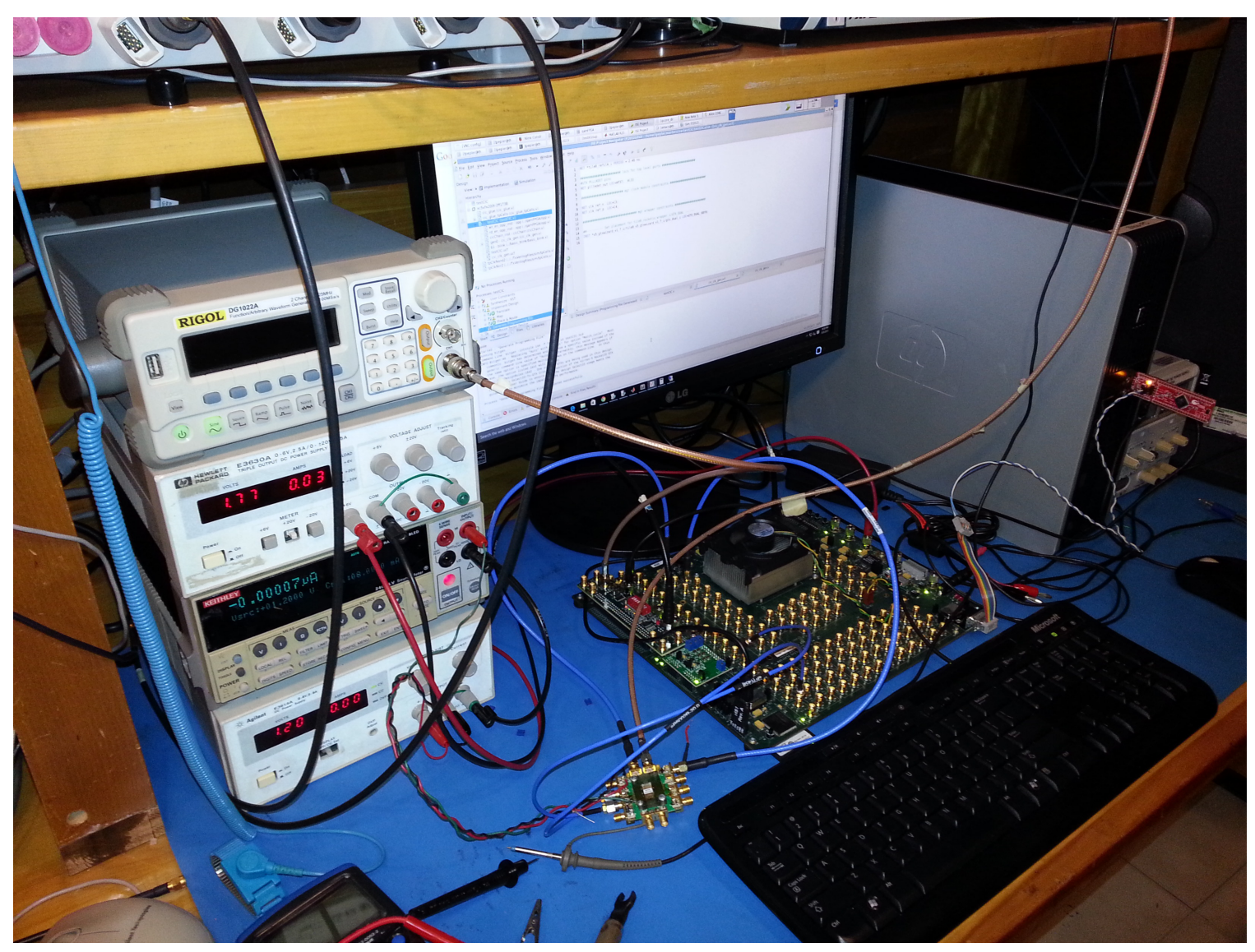

Figure 4.4: Laboratory bench setup for testing the CIC filter. 


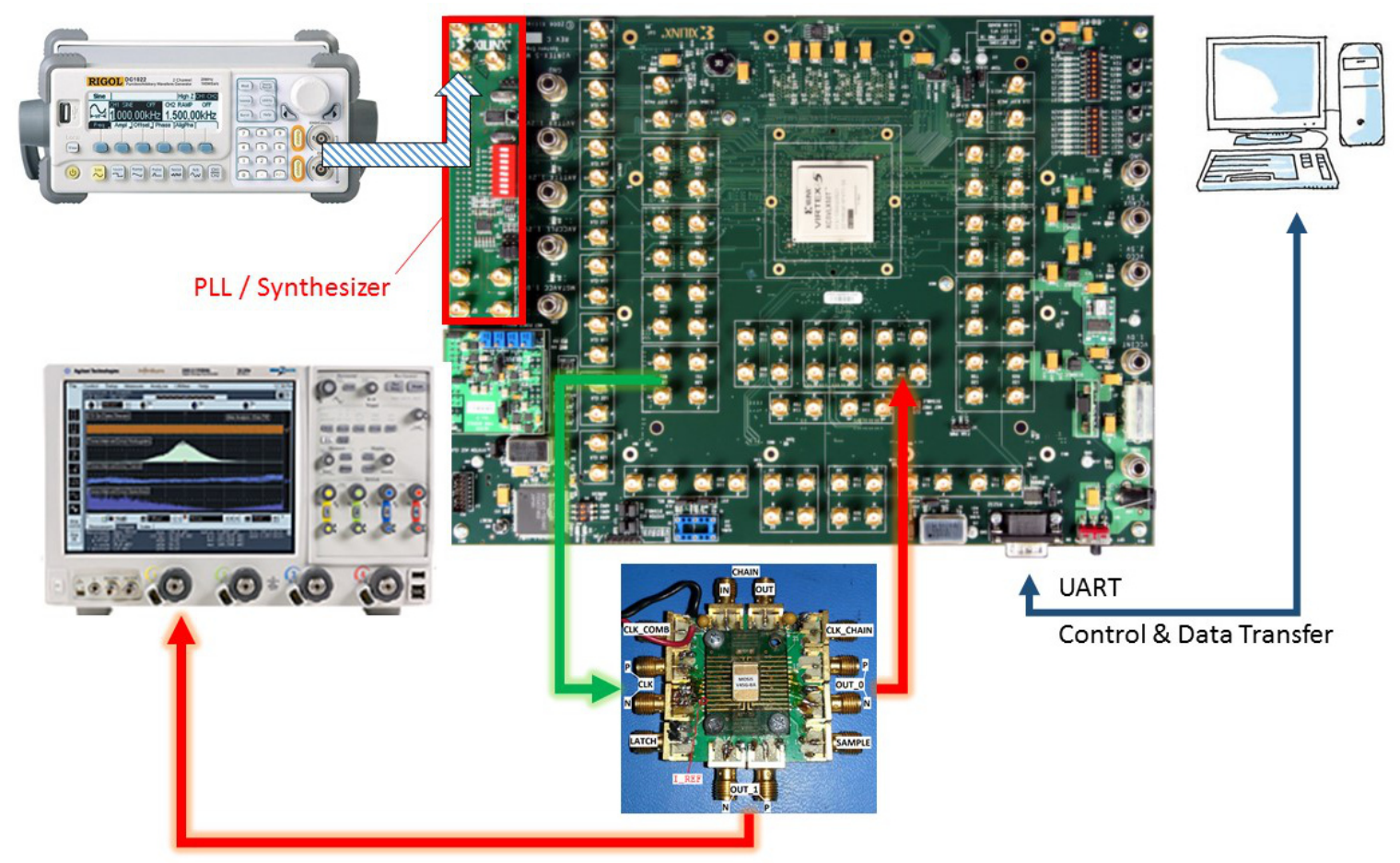

Figure 4.5: Schematic showing the test setup for the CIC filter.

and also to have non-overlapping clocks, either by designing skew into the buffers as was done for the high-speed clock net, or implementing a two-phase non-overlapping clock generator circuit. Due to the constrained number of pads, the option of feeding in both clock phases from off-chip was not available (although this would have been the safest option, as it can provide the most control over the amount of non-overlap for the phases).

Unfortunately, due to the scan chain problem it was not possible to load values into the CIC filter, or read values out; therefore the operation of the filter could not be verified.

\subsection{QAM Tx}

Testing of the QAM transmitter on the FPGA used a very similar setup to the CIC chip. The 6 Gbps FPGA transmitters are routed to SMA connectors on the board. SMA terminated coaxial cables were used to connect the output of the FPGA to the Agilent DSA.

The data output of the QAM modulator is sent serially out of the FPGA, and the voltage waveform representing the stream of bits was captured with the DSA. 
Name

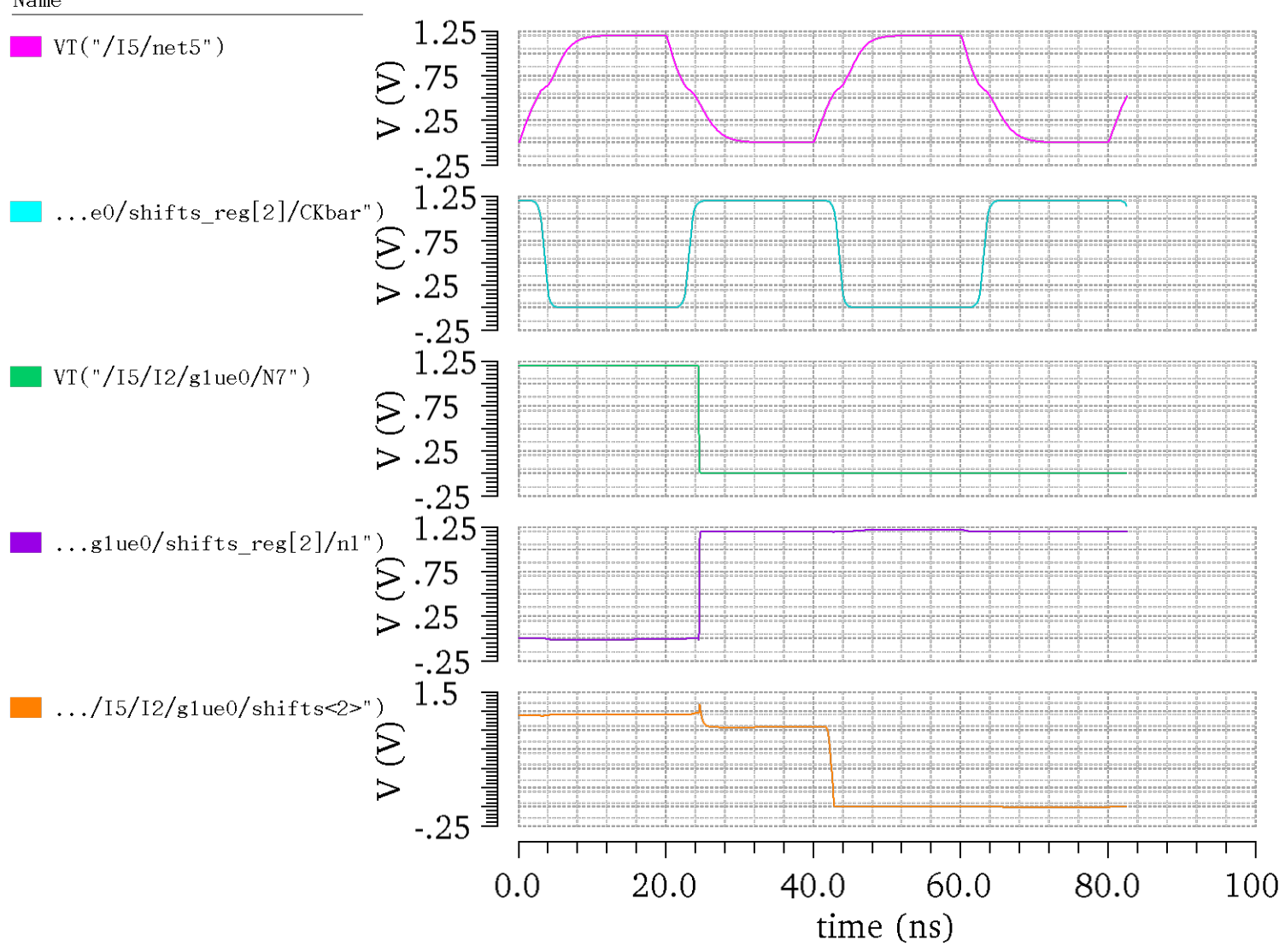

Figure 4.6: Plot of the simulation of the scan chain signals, showing the much slower rise time of $c l k$ compared to $c l k_{b}$.

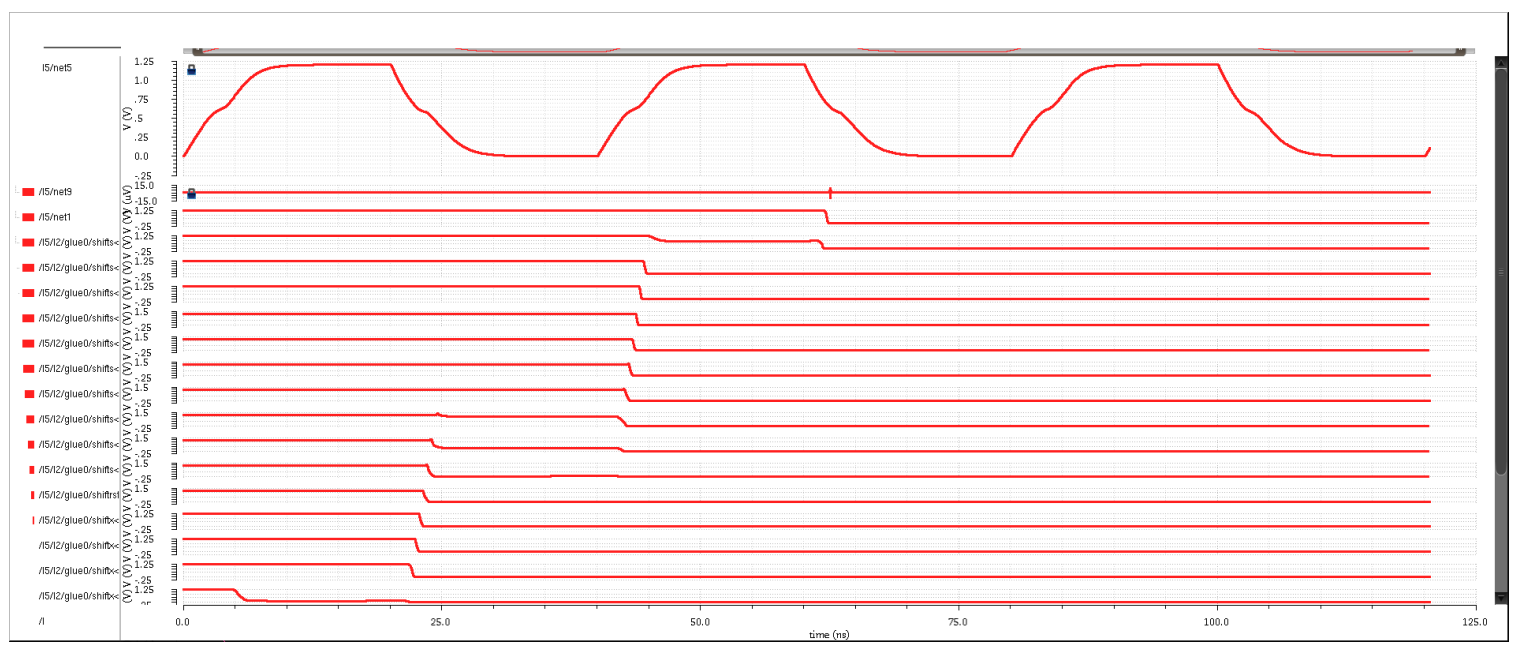

Figure 4.7: Plot of the clock signal and all of the outputs of the scan chain FFs. 
The voltage waveform is then decoded into the actual sequence of bits. This was accomplished using the built-in serial decode function of the DSA. The bits were then loaded into MATLAB, converted to the appropriate fixed point values, and the values compared with the original MATLAB simulation results.

The FPGA functioned correctly up to a maximum logic frequency of $548 \mathrm{MHz}$.

For example, a plot of the QAM modulator output for a pseudo-random stream of bits is compared to the input symbol values, showing that the pulse shaping filter is working correctly, as the two sets of data coincide at the correct sampling point every symbol period. This is shown in Figure 4.8 .

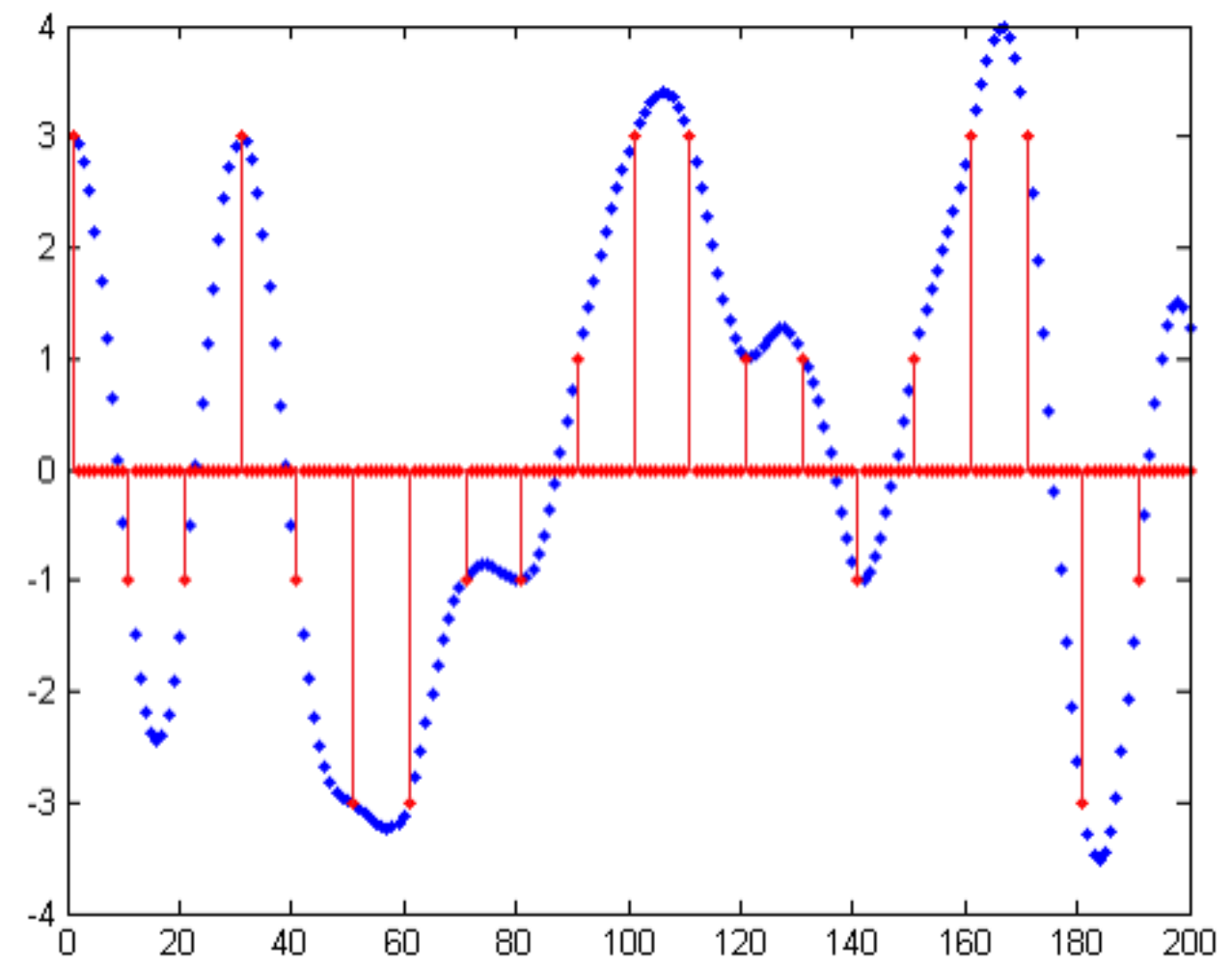

Figure 4.8: Plot of the output of the QAM modulator (blue), and the ideal symbol values (red, with stems).

\subsection{Summary}

This chapter discussed the results of testing the various designs in the lab. The first test chip of buffers was found to be functional, but at a lower maximum frequency 
than desired. The second test chip, unfortunately did not function due to an error overlooked in the design process. The QAM modulator implemented on an FPGA worked well, achieving a maximum clock frequency of $548 \mathrm{MHz}$. The next chapter will conclude the thesis with a summary, and provide recommendations for future work. 


\section{Chapter 5}

\section{Conclusion}

This chapter will provide a summary of the thesis and provide some suggestions for future work.

\subsection{Summary}

The objectives of this thesis were to develop an algorithm for automatically pipelining a register-transfer level digital design, and to demonstrate the technique with a CMOS ASIC and an FPGA. Additionally, the ASIC used the less conventional logic family known as Clocked CMOS to improve the area and power efficiency compared to the traditional pipelining approach of inserting flip-flops between logic stages.

First, a background on the relevant theory for the designs was given. Then the details of the design process for the various elements of the research was given. Finally, the results of testing the ASICs and FPGA in the lab were presented.

\subsection{Contributions}

The following is a list of the contributions in this thesis:

- Pipelining and delay balancing algorithms were developed and implemented in Perl. The Perl implementation uses the Graph module described in [10] and available from [27] to implement the depth-first search part of the algorithm.

- The delay balancing algorithm was applied to a $\mathrm{C}^{2} \mathrm{MOS}$ logic circuit to automatically balance the cycle delays along all input-to-output paths. The $\mathrm{C}^{2} \mathrm{MOS}$ logic style was first proposed by Suzuki, et al. in 1973 [1]. 
- The pipelining algorithm was applied to a 16-QAM modulator logic circuit. The resulting pipelined circuit was synthesized and successfully demonstrated on an FPGA, which achieved a maximum clock rate of $548 \mathrm{MHz}$.

\subsection{Future Work}

Unfortunately, for the ASIC, due to an error in the design of the scan chain, the chip could not be verified in the lab.

The following are suggestions for future work that would allow the techniques used in this thesis to be further verified. These changes are based on some problems uncovered during the design of the CIC filter.

- The scan chain on the CIC filter chip should be redesigned to buffer the clock net, and ensure non-overlapping clocks. Alternatively, a more conventional flipflop style that only requires a single clock phase could be used.

- A better clock distribution strategy should be developed. More investigation needs to be done to make a better clock buffer cell and to have Cadence Encounter automatically place them at optimal locations. 


\section{List of References}

[1] V. Suzuki, K. Odagawa, and T. Abe, "Clocked CMOS calculator circuitry," Solid-State Circuits, IEEE Journal of, vol. 8, no. 6, pp. 462-469, December 1973.

[2] D. Stranneby and W. Walker, Digital Signal Processing and Applications, 2nd ed. Elsevier Science, 2004.

[3] J. Proakis and D. Manolakis, Digital Signal Processing: Principles, Algorithms, and Applications, 3rd ed. Upper Saddle River, N.J: Prentice Hall, 1996.

[4] L. C. Ludeman, Fundamentals of Digital Signal Processing. New York: Harper \& Row, 1986.

[5] S. W. Smith, The Scientist and Engineer's Guide to Digital Signal Processing. San Diego, CA, USA: California Technical Publishing, 1997.

[6] f. harris, Multirate Signal Processing for Communication Systems. Upper Saddle River, N.J: Prentice Hall PTR, 2004.

[7] E. Hogenauer, "An economical class of digital filters for decimation and interpolation," Acoustics, Speech and Signal Processing, IEEE Transactions on, vol. 29, no. 2, pp. 155-162, April 1981.

[8] E. C. Ifeachor and B. W. Jervis, Digital Signal Processing: A Practical Approach, 2nd ed. Prentice Hall, 2002, ch. 9.

[9] Y. J. Yu, Y. C. Lim, and T. Saramaki, "Restoring coefficient symmetry in polyphase implementation of linear-phase FIR filters," Circuits, Systems and Signal Processing, vol. 25, no. 2, pp. 253-264, 2006. [Online]. Available: http://dx.doi.org/10.1007/s00034-005-2506-4

[10] J. Orwant, J. Hietaniemi, and J. Macdonald, Mastering Algorithms with Perl, 1st ed. Sebastopol, CA: O’Reilly, 1999.

[11] J. Rogers, C. Plett, and I. Marsland, Radio Frequency System Architecture and Design. Boston: Artech House, 2013.

[12] E. McCune, Practical Digital Wireless Signals, ser. The Cambridge RF and Microwave Engineering Series. Cambridge University Press, 2010.

[13] L. W. Couch, Digital and Analog Communication Systems. Upper Saddle River, N.J: Pearson Prentice Hall, 2007.

[14] N. Weste and D. Harris, CMOS VLSI Design: A Circuits and Systems Perspective, 4th ed. Addison Wesley, 2011. 
[15] B. Yu and M. L. Bushnell, "Power grid analysis of dynamic power cutoff technology," in 2007 IEEE International Symposium on Circuits and Systems, May 2007, pp. 1393-1396.

[16] J. Rabaey, Low Power Design Essentials, ser. Integrated Circuits and Systems. Springer Science \& Business Media, 2009.

[17] J. Rabaey, A. Chandrakasan, and B. Nikolic, Digital Integrated Circuits: A Design Perspective, 2nd ed., ser. Prentice Hall electronics and VLSI series. Pearson Education, 2003.

[18] (2016) Make - CMC Microsystems. [Online]. Available: http://www.cmc.ca/ WhatWeOffer/Make.aspx

[19] C. E. Leiserson and J. B. Saxe, "Retiming synchronous circuitry," Algorithmica, vol. 6, no. 1, pp. 5-35, 1991.

[20] "HDL Coding Practices to Accelerate Design Performance," White Paper, Xilinx, January 2006.

[21] "AN715: Hyper-Pipelining for Stratix 10 Designs," Application Note, Altera, June 2015.

[22] A. El-Maleh, T. E. Marchok, J. Rajski, and W. Maly, "Behavior and testability preservation under the retiming transformation," IEEE Transactions on Computer-Aided Design of Integrated Circuits and Systems, vol. 16, no. 5, pp. 528-543, May 1997.

[23] "SIV5V1: Stratix IV Device Handbook: Volume 1," Handbook, Altera, September 2012.

[24] "UG190: Virtex-5 FPGA User Guide," User Guide, Xilinx, March 2012.

[25] (2016) The Comprehensive Perl Archive Network. [Online]. Available: http://www.cpan.org/

[26] (2016) Intro - Verilog-Perl - Veripool. [Online]. Available: http://www.veripool. org/wiki/verilog-perl

[27] (2016) Jarkko Hietaniemi / Graph-0.9704 - search.cpan.org. [Online]. Available: http://search.cpan.org/ jhi/Graph-0.9704/

[28] (2016) Manual-verilog-perl - Verilog-Perl - Veripool. [Online]. Available: http://www.veripool.org/projects/verilog-perl/wiki/Manual-verilog-perl

[29] R. Baker, CMOS: Circuit Design, Layout, and Simulation, ser. IEEE Press Series on Microelectronic Systems. Wiley, 2010, sec. 11.4.

[30] S. Voinigescu, High-Frequency Integrated Circuits, ser. High-frequency Integrated Circuits. Cambridge University Press, 2013, sec. 12.4.

[31] IEEE Standard for Inform. technol.-Telecommun. and inform. exchange between syst.-Local and metropolitan area networks-Specific requirements-Part 11: Wireless LAN Medium Access Control (MAC) and Physical Layer (PHY) Specifications Amendment 3: Enhancements for Very High Throughput in the $60 \mathrm{GHz}$ Band, IEEE Std. 802.11ad-2012, 2012. 
[32] E. Perahia and M. X. Gong, "Gigabit wireless LANs: An overview of IEEE 802.11ac and 802.11ad," SIGMOBILE Mob. Comput. Commun. Rev., vol. 15, no. 3, pp. 23-33, Nov. 2011. [Online]. Available: http: //doi.acm.org/10.1145/2073290.2073294

[33] 3rd Generation Partnership Project; Technical Specification Group Radio Access Network; Base Station (BS) radio transmission and reception (FDD) (Release 6), 3GPP Std. TS 25.104 V6.8.0, 2004. 